\title{
A simpler algorithm and shorter proof for the graph minor decomposition
}

\author{
Ken-ichi Kawarabayashi * \\ National Institute of Informatics \\ 2-1-2 Hitotsubashi, Chiyoda-ku \\ Tokyo 101-8430, Japan \\ k_keniti@nii.ac.jp
}

\author{
Paul Wollan \\ Department of Computer Science \\ University of Rome, La Sapienza \\ Via Salaria 113 \\ Rome, 00198 Italy \\ wollan@di.uniroma1.it
}

\begin{abstract}
At the core of the Robertson-Seymour theory of graph minors lies a powerful decomposition theorem which captures, for any fixed graph $H$, the common structural features of all the graphs which do not contain $H$ as a minor. Robertson and Seymour used this result to prove Wagner's Conjecture that finite graphs are well-quasi-ordered under the graph minor relation, as well as give a polynomial time algorithm for the disjoint paths problem when the number of the terminals is fixed. The theorem has since found numerous applications, both in graph theory and theoretical computer science. The original proof runs more than 400 pages and the techniques used are highly non-trivial.

Robertson and Seymour's proof yields an $O\left(n^{3}\right)$-time algorithm to find the decomposition. In this paper, we give a simplified algorithm for finding the decomposition based on a new constructive proof of the decomposition theorem for graphs excluding a fixed minor $H$. The new proof is both dramatically simpler and shorter, making these results and techniques more accessible. The algorithm runs in time $O\left(n^{3}\right)$ as well. Moreover, our proof gives an explicit bound on the constants in the $O$-notation.
\end{abstract}

\section{Introduction}

A graph $H$ is a minor of a graph $G$ if $H$ can be obtained from a subgraph of $G$ by contracting edges. The theory of graph minors was developed by Robertson and Seymour in a series of 23 papers published over more than twenty-five years. The aim of the series of papers is to prove a single result: the graph minor theorem, which says that in any infinite collection of finite graphs there is one that is a minor of another. As with other deep results in mathematics, the body of theory developed for the proof of the graph minor theorem has also found applications elsewhere, both within graph theory and computer science. Yet many of these applications rely not only on the general techniques developed by Robertson and Seymour to handle graph minors, but also on one particular auxiliary result which is central to the proof of the graph minor theorem: a result which approximately describes the structure of all graphs $G$ which do not contain some fixed graph $H$ as a minor. At a high level, the theorem says that every such a graph can be decomposed into a collection of graphs each of which can be "almost" embedded into a bounded-genus surface; the pieces can be assembled in a tree structure to obtain the original graph. This decomposition theorem is used to verify Wagner's Conjecture [41], which can be stated as follows: every minor-closed graph property (i.e. a property preserved under taking of minors) is characterized by a finite set of forbidden minors. It is also used in the proof of the correctness for the seminal graph minor algorithm, a polynomial-time algorithm for testing the

* Research partly supported by Japan Society for the Promotion of Science, Grant-in-Aid for Scientific Research, by C \& C Foundation, by Kayamori Foundation and by Inoue Research Award for Young Scientists. 
presence of a fixed minor [36]. When combined together, the graph minor algorithm and the proof of Wagner's Conjecture immediately imply the existence of a polynomial-time algorithm for deciding membership in any minor-closed class of graphs. There have been numerous applications of the decomposition theorem; we discuss this more thoroughly below.

The proof of this decomposition theorem is extremely long and technical, and utilizes much of the theory of graph minors which Robertson and Seymour developed. The proof occupies the first 16 graph minor papers and is at least 400 pages long. Moreover, some of the bounds given in the proof are not explicit. The combination of the usefulness of the graph minor decomposition theorem and the difficulty of the proof have motivated the search for a more accessible proof. To quote a survey article of Lovász' [24], "It would be quite important to have simpler proofs with more explicit bounds. Warning: many of us have tried, but only a few successes can be reported."

\subsection{Our contribution}

Building on the methods we have developed in [17], we give a much shorter proof of the graph minor decomposition theorem. We begin by giving the necessary notation to state the decomposition theorem more precisely.

A path decomposition of a graph $G$ consists of linearly ordered subsets $\mathcal{B}_{i}, 1 \leq i \leq k$ with $\mathcal{B}_{i} \subseteq V(G)$ such that for every edge $u v \in E(G)$ there exists an index $i$ such that $u, v \in \mathcal{B}_{i}$ and furthermore, for all vertices $x$, if $x \in \mathcal{B}_{i}$ and $x \in \mathcal{B}_{j}$, then $x \in \mathcal{B}_{l}$ for all $i \leq l \leq j$. The width of the decomposition is the maximal size of a $\mathcal{B}_{i}$, and the path-width of a graph $G$, denoted $\operatorname{pw}(G)$, is the minimum width over all possible path decompositions of $G$. The adhesion of the decomposition is the $\max _{1 \leq i<j \leq k} \mathcal{B}_{i} \cap \mathcal{B}_{j}$.

In this paper, an embedding refers to a 2-cell embedding, i.e. a drawing of the vertices and edges of the graph as points and arcs in a surface such that every face (region outlined by edges) is homeomorphic to a disc. A non-contractable curve in the surface is simply a curve that cannot be contracted continuously to a point on the surface. The representativity, or face-width, of an embedded graph is minimum number of times a non-contractable curve $C$ intersects the embedded graph, with the minimum taken over all such non-contractable curves $C$.

Let $G$ be a graph and $\Sigma$ be a general surface. Let $k$ be a positive integer. A $k$-near embedding in $\Sigma$ consists of edge disjoint subgraphs $H_{0}, H_{1}, \ldots, H_{m}$ for some positive integer $m$ satisfying the following conditions.

i. $\bigcup_{i=0}^{m} H_{i}=G$.

ii. For all $i, j \geq 1, i \neq j, V\left(H_{i}\right) \cap V\left(H_{j}\right) \subseteq V\left(H_{0}\right)$.

iii. For all $i>k,\left|V\left(H_{i}\right) \cap V\left(H_{0}\right)\right| \leq 3$.

iv. There exist pairwise disjoint open discs $\Delta_{1}, \ldots, \Delta_{m}$ and an embedding $\sigma: H_{0} \hookrightarrow \Sigma-\bigcup_{i=1}^{m} \Delta_{i}$ such that the only vertices contained in the boundary of $\Delta_{i}$ are exactly the vertices of $H_{i} \cap H_{0}$ for $i=1, \ldots, m$.

v. For $1 \leq i \leq k$, let the vertices of $V\left(H_{i}\right) \cap V\left(G_{0}\right)$ be $u_{1}, u_{2}, \ldots, u_{n}$ for some integer $n$ with the order given by their order on the boundary of the disc $\Delta_{i}$ in $\Delta$. Then the graph $H_{i}$ has a path decomposition $\left(\mathcal{B}_{j}\right)_{1 \leq j \leq n}$ such that $u_{j} \in \mathcal{B}_{j}$ for all $1 \leq j \leq n$.

The $k$-near embedding is $\alpha$-bounded if the path decomposition of $H_{i}$ in $\mathrm{v}$. has adhesion at most $\alpha$ for all $1 \leq i \leq k$. The $k$-near embedding is totally bounded if both $m \leq k$ and the path decomposition of $H_{i}$ in $v$ has width at most $k$ for all $1 \leq i \leq k$. Finally, a graph $G$ is $t$-close to admitting a $k$-near embedding if there exists a set $X \subseteq V(G)$ with $|X| \leq t$ such that $G-X$ admits a $k$-near embedding.

The pieces of the decomposition are combined according to "clique-sum" operations, a notion which goes back to characterizations of $K_{5}$-minor-free graphs by Wagner [48]. Suppose $G_{1}$ and $G_{2}$ are graphs with 
disjoint vertex sets and let $k \geq 0$ be an integer. For $i=1,2$, let $W_{i} \subseteq V\left(G_{i}\right)$ form a clique of size $k$ and let $G_{i}^{\prime}$ be obtained from $G_{i}$ by deleting some (possibly no) edges from the induced subgraph $G_{i}\left[W_{i}\right]$. Consider a bijection $h: W_{1} \rightarrow W_{2}$. We define a $k$-sum $G$ of $G_{1}$ and $G_{2}$, denoted by $G=G_{1} \oplus_{k} G_{2}$, to be the graph obtained from the union of $G_{1}^{\prime}$ and $G_{2}^{\prime}$ by identifying $w$ with $h(w)$ for all $w \in W_{1}$. The images of the vertices of $W_{1}$ and $W_{2}$ in $G_{1} \oplus_{k} G_{2}$ form the join set. Note that each vertex $v$ of $G$ has a corresponding vertex in $G_{1}$ or $G_{2}$ or both. It is also worth mentioning that $\oplus$ is not a well-defined operator: it can have a set of possible results.

Now we can state a precise form of the decomposition theorem:

Theorem 1.1 (Decomposition Theorem, Theorem 1.3 [39] ) For every graph H, there exists an integer $h \geq 0$ depending only on $|V(H)|$ such that every $H$-minor-free graph can be obtained by at most $h$-sums of graphs which are $h$-close to admiting a totally bounded h-near embedding in some surfaces in which $H$ cannot be embedded.

We give a shorter and simpler proof of Theorem 1.1. The proof is constructive and immediately yields an $f(|H|) n^{3}$ time algorithm to find such a decomposition for excluding $H$-minor. In addition, our proof also gives an explicit bound for $h$.

The original proof of Robertson and Seymour also gives an $f^{\prime}(|H|) n^{3}$ algorithm to find the decomposition, if one follows all the arguments very carefully (as pointed out by Reed (private communication)). Our algorithm is an improvement, in that it is far easier and more accessible, and in that it improves the bounds on the function $f$ and gives explicit bounds for the value $h$. Recently, Reed, Li and the first author announced an $O(n \log n)$ algorithm to find the decomposition theorem. The proof generally follows the argument in the graph minor theory, however some of the more technical graph minor results must be strengthened. Complete details will require more than 100 pages and are not yet fully written down.

\section{$1.2 \quad$ Algorithmic applications}

Algorithms for $H$-minor-free graphs for a fixed graph $H$ have been studied extensively; see e.g. [4, 5, 13, 21, 27]. In particular, it is generally believed that several algorithms for planar graphs can be generalized to $H$-minor-free graphs for any fixed $H[13,21,27]$. The decomposition theorem provides the key insight into why this might be possible: given an algorithm for planar graphs, first extend it to handle bounded-genus graphs; then extend it further to handle graphs "almost-embeddable" into bounded-genus surfaces, and finally generalize the results to resolve the problem on tree decompositions into graphs almost-embeddable in bounded genus surfaces. The graph minor decomposition theorem has already been used to obtain many combinatorial results and show the existence of many efficient algorithms, despite being published only recently. Grohe [12] proves the existence of PTASs for minimum vertex cover, minimum dominating set, and maximum independent set in $H$-minor-free graphs. However, such an approach requires an algorithm to construct the decomposition. Our simpler proof and algorithm gives a fast and more accessible algorithm for these problems.

DeVos et al [9] used the decomposition theorem to prove that for every integer $k \geq 1$ and every fixed graph $H$, every $H$-minor-free graph has a vertex partition into parts $V_{1}, \ldots, V_{k}$ and edge partition $E_{1}, \ldots, E_{k}$ such that for every $i \in\{1, \ldots, k\}$, the graphs $G-V_{i}$ and $G-E_{i}$ have bounded treewidth. It follows that given an algorithm to construct the decomposition, there is a 2-approximation algorithm for graph coloring in minor-closed class of graphs, see [8]. A special case of this partitioning result restricted to planar graphs was proved by Baker [2] who used it to devise efficient approximation algorithms (and approximation schemes) for several hard approximation algorithms on planar graphs. Baker used the planar separator theorem of Lipton and Tarjan [23]. Alon, Seymour, and Thomas [1] proved a similar separator theorem for graphs excluding any fixed minor, and this result is further generalized by Kawarabayashi and Reed [19]. Eppstein [11] extended Baker's ideas to graphs in arbitrary proper minor-closed classes of graphs. Again, these methods for finding approximation algorithms for problems in minor closed classes begin with the graph minor decomposition. Again, our proof and algorithm yields fast and more accessible algorithms for these approximation results. 
Demaine et al [6] have been working on directly using the graph minor theorem in algorithmic applications. They obtained subexponential fixed-parameter algorithms for dominating set, vertex cover and set cover in any class of graphs excluding a fixed graph $H$ as a minor. Specifically, the running time is $2^{O(\sqrt{k})} n^{h}$, where $h$ is a constant depending only on $H$. For further applications, see the survey [7] or the paper [8]. Our simpler proof gives not only more accessible and simpler algorithms but also faster algorithms, i.e. $h=3$.

\section{The proof and algorithm}

Although the decomposition theorem is the one that appears now to be best known, and which has also found the most algorithmic applications, Robertson and Seymour themselves [39] later dubbed it a 'red herring' in the search for the proof of the graph minor theorem. We begin this section by describing the "main" structure theorem in graph minor theory.

A particularly simple form of this structure theorem applies when the excluded minor $H$ is planar: in that case, the said parts of $G$ - the parts that fit together in a tree-structure and together make up all of $G$-have bounded size, i.e., $G$ has bounded treewidth. If $H$ is not planar, the graphs $G$ not containing $H$ as a minor have unbounded treewidth, and therefore contain arbitrarily large grids as minors and arbitrarily large walls as a topological minor. Such a large grid or wall identifies, for every low-order separation of $G$, one side in which most of that grid or wall lies. This is formalized by the notion of a tangle: the larger the treewidth of $G$, the larger the grid or wall, the order of the separations for which this works, and (thus) the order of the tangle. Since adjacent parts in our tree decomposition of $G$ meet in only a bounded number of vertices and thus define low-order separations, our large-order tangle 'points to' one of the parts, the part $G^{\prime}$ that contains most of its defining grid or wall. This allows Robertson and Seymour to focus on one piece of the tree decomposition. In fact, most of the details of the graph minor papers are devoted to prove the structure theorem capturing our large-order tangle, which we shall call the "main" structure theorem of the graph minor theory.

We are now ready to state the main structure theorem in graph minor theory

Theorem 2.1 (Structure Theorem, [39]) For every graph $R$ there exist integers $\theta, \alpha=\alpha(|R|) \geq 0$ such that the following holds: Let $G$ be a graph that does not contain $R$ as a minor and $\mathcal{T}$ be a tangle in $G$ of order at least $\theta$. Then there exists a subset $A \subseteq V(G)$ with $|A| \leq \alpha$ such that $G-A$ has an $\alpha$-bounded $\alpha$-near embedding into a surface $\Sigma$ in which $R$ cannot be drawn. Moreover, this near embedding captures $\mathcal{T}-A$.

Assuming Theorem 2.1, the decomposition theorem can be proven relatively easily. We give the proof in Section 3. Thus, the majority of the work in our proof of the decomposition theorem lies in proving Theorem 2.1. Our main tool in proving Theorem 2.1 will be several new results on embedding societies in surfaces. We discuss this in more detail in the next subsection. We then give an outline of the proof of Theorem 2.1 in Subsection 2.2.

\subsection{Nearly embedding a society}

A society is a pair $(G, \Omega)$ where $G$ is a graph and $\Omega$ is a cyclic ordering of some of the vertices of $G$. Societies play a key role in the graph minor series, see [32].

In our proof, we will need to understand when a given society $(G, \Omega)$ is $t$-close to having a $t$ bounded $k$-near embedding in the disc $\Delta$ for given values $t$ and $k$. Two possible obstructions are what's known as $k$-crosscaps and $k$-handles. A $k$-crosscap consists of $k$ disjoint, pairwise crossing paths with their endpoints in $\Omega$. A $k$-handle consists of $2 k$ pairwise disjoint paths $P_{1}, \ldots, P_{k}, Q_{1}, \ldots, Q_{k}$ each with their endpoints in $\Omega$ which satisfy the following. For all $i, j$, we have that $P_{i}$ and $P_{j}$ do not cross and similarly, $Q_{i}$ and $Q_{j}$ do not cross, and alternatively, the paths $P_{i}$ and $Q_{j}$ do cross. See Figure 1. 

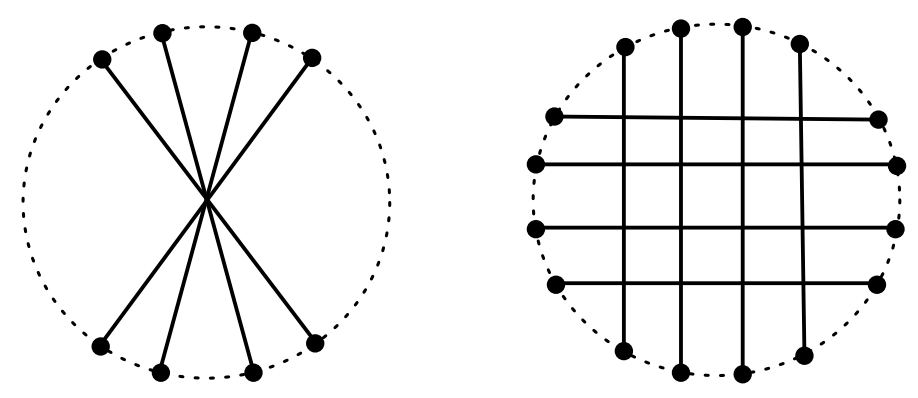

Figure 1: An example of a 4-crosscap and a 4-handle.

It would be convenient if these were the only obstructions. Unfortunately, we must define several patterns consisting of disjoint paths with their endpoints in $\Omega$. For the exact definition, we refer to the appendix and here denote the pattern with $k$ paths as $\mathcal{P}_{k}$.

Theorem 2.2 Let $k \geq 1$ be given. There exists a value $\alpha=\alpha(k)$ such that for every society $(G, \Omega)$, one of the following holds.

(i) $(G, \Omega)$ contains a $k$-crosscap, a $k$-handle, or the pattern $\mathcal{P}_{k}$.

(ii) $(G, \Omega)$ is $\alpha$-close to admitting an $\alpha$-bounded $\alpha$-near embedding in the disc $\Delta$ such that all the vertices of $\Omega$ are embedding on the boundary of $\Delta$ in the order indicated by $\Omega$

Theorem 2.2 generalizes the main theorem of [32]. Let $\Delta$ be the disc and let $D_{1}$ and $D_{2}$ be two disjoint, open discs in $\Delta$ which do not intersect the boundary of $\Delta$. Let $\Sigma_{1}$ be the surface obtained by deleting $D_{1}$ and gluing a crosscap to the boundary of $D_{1}$. Let $\Sigma_{2}$ be the surface obtained by deleting $D_{1}$ and $D_{2}$ from $\Delta$ and gluing a handle onto the boundaries of $D_{1}$ and $D_{2}$. The existence of a $k$-crosscap or $k$-handle for large $k$ in a society $(G, \Omega)$ implies that the natural surface in which we should attempt to embed $(G, \Omega)$ is $\Sigma_{1}$, or $\Sigma_{2}$, respectively. When we can do so is described in the following theorem.

Theorem 2.3 Let $k \geq 1$ be given. There exists a value $f=f(k)$ and $\alpha=\alpha(k)$ satisfying the following. Let $(G, \Omega)$ be a society containing a $f$-crosscap (f-handle) $\mathcal{Q}$ but which does not contain the pattern $\mathcal{P}_{k}$. Then there exists a set $X \subseteq V(G)$ with $|X| \leq \alpha$ and edge disjoint subgraphs $H_{1}$ and $H_{2}$ of $G$ such that $H_{1} \cup H_{2}=G-X$ which satisfy the following.

(i) $H_{1}$ contains a $k$-crosscap ( $k$-handle) $\mathcal{Q}^{\prime}$ which is a subgraph of $\mathcal{Q}$.

(ii) $\Omega-X \subseteq V\left(H_{1}\right)$ and $\left(H_{1}, \Omega-X\right)$ admits a 0 -near embedding in $\Sigma_{1}\left(\Sigma_{2}\right)$.

(iii) There exists a single face of the near embedding in $\Sigma_{1}$ or $\Sigma_{2}$ which contains all the vertices of $V\left(H_{1}\right) \cap$ $V\left(H_{2}\right)$.

The proofs of Theorems 2.2 and 2.3 contain the main technical work in the new proof. Interested readers are directed to the appendix for the full proofs. We will see in the next subsection how these theorems come into the proof of Theorem 2.1.

\subsection{Outline of the proof of Theorem 2.1}

We now give an overview of our proof for the structure theorem, Theorem 2.1. Our starting point is so called "Weak Structure Theorem" which is proved in Graph Minor XIII [36]. Roughly it says that, given any grid 
or wall $W$ of size $f(t, k)$ in a given graph $G$, either $G$ has a $K_{t}$-minor, or $G$ has a vertex set $Z$ of order at most $t^{2}$ such that $G-Z$ has a subwall $W^{\prime}$ of $W$ of size $k$ which induces "essentially" planar embedding in $G-Z$. More explicitly:

Theorem 2.4 (Weak Structure Theorem, [36]) Let $t$ and $l$ be given. There exists $f=f(t, l)$ satisfying the following. Let $G$ be a graph containing a wall $W$ of size $f$. Then either $G$ contains a $K_{t}$ minor which cannot be separated from $W$ by a small order separation, or there exists a subset $A \subseteq V(G)$, a sub-wall $W^{\prime}$ of $W$ of size $l$ and edge disjoint subgraphs $H_{1}$ and $H_{2}$ of $G$ satisfying the following.

(i) $|A| \leq t^{2}$ and $G-A=H_{1} \cup H_{2}$.

(ii) $W^{\prime}$ is a subgraph of $H_{1}$

(iii) $H_{1}$ has a 0-near embedding in the disc $\Delta$ such that $W^{\prime}$ is not separated from $\Delta$ by a small separation and all the vertices of $V\left(H_{1}\right) \cap V\left(H_{2}\right)$ are embedding on the boundary of $\Delta$.

Note that given the structure in Theorem 2.4, we have a natural society given by the subgraph $H_{2}$ and the cyclic ordering of the vertices of $V\left(H_{1}\right) \cap V\left(H_{2}\right)$ given by their embedding on the boundary of the disc $\Delta$.

We begin with a graph $G$ not containing $K_{t}$ as a minor, and now our proof adapts some ideas in [38, 39] to grow a large subgraph of $G$ which essentially embeds on a surface with large representativity. We maintain two subgraphs $H_{1}$ and $H_{2}$ of $G$ and a subset of the vertices $Z$ such that

1. $H_{1} \cup H_{2}=G-Z$,

2. $H_{1}$ essentially embeds in a surface $\Sigma$ with big representativity (technically, $H_{1}$ has a 0 -near embedding in $\Sigma$ ), and

3. there is a single face $F$ of the embedding of $H_{1}$ containing the vertices of $V\left(H_{1}\right) \cap V\left(H_{2}\right)$.

We then consider the society given by $H_{2}$ and the cyclic order of the vertices of $V\left(H_{1}\right) \cap V\left(H_{2}\right)$ given by the boundary of the face $F$ (denote as $\Omega$ this cyclic order of $V\left(H_{1}\right) \cap V\left(H_{2}\right)$ ). If there existed a set $Z_{2} \subseteq V\left(H_{2}\right.$ ) with $\left|Z_{2}\right| \leq \alpha$ such that $\left(H_{2}-Z_{2}, \Omega-Z_{2}\right)$ admitted an $\alpha$-bounded $\alpha$-near embedding of $(G, \Omega)$ in the disc, then we could "glue" it onto the embedding of $H_{1}$ in $\Sigma$ and find the desired $\alpha$-bounded $\alpha$-near embedding of $G-\left(Z \cup Z_{2}\right)$.

We apply Theorem 2.2 to attempt to find such a nice embedding of $\left(H_{2}, \Omega\right)$. We can (by picking $\alpha$ sufficiently large), assume that we find either a large crosscap or handle, or a large pattern $\mathcal{P}_{k}$. However, it is straightforward to show that if $k$ is large, then $H_{1}$ along with the pattern $\mathcal{P}_{k}$ yields a large clique minor. Thus, we may assume that we always find a large crosscap or handle. We apply Theorem 2.3 to the society $\left(H_{2}, \Omega\right)$ along with the large crosscap or handle. We find subgraphs $J_{1}$ and $J_{2}$ and subset $X$ of vertices such that $\left(J_{1}, \Omega-X\right)$ embeds in the disc plus a crosscap or handle.

Returning to our graph original graph $G$, we see that $G-(Z \cup X)=H_{1} \cup J_{1} \cup J_{2}$ and that $H_{1} \cup J_{1}$ has a 0-near embedding in the surface $\Sigma^{\prime}$ obtained by adding a single crosscap or handle to $\Sigma$. Moreover, by using the fact that $\left(J_{1}, \Omega-X\right)$ contains a large handle or crosscap, we can ensure that this embedding of $H_{1} \cup J_{1}$ embeds with large representativity (although it will be somewhat smaller than the representativity of the embedding of $H_{1}$ in $\Sigma$ ). Finally, there exists a single face of the embedding in $\Sigma^{\prime}$ which contains all the vertices of $V\left(H_{1} \cup J_{!}\right) \cap V\left(J_{2}\right)=V\left(J_{1}\right) \cap V\left(J_{2}\right)$.

Thus, at each inductive step we grow the genus of the surface maintaining large representativity. The base case in the induction is the weak structure theorem with the large graph essentially embedded in the sphere. In this special case, the wall plays the role of representativity. The whole process must eventually stop since there is a theorem [30] that states that for a sufficiently large genus surface and a sufficiently large amount of representativity, any graph embedded with such representativity must contain $K_{t}$ as a minor. 


\subsection{Improvements over the original}

Let us clarify why our proof is shorter than the original proof by Robertson and Seymour.

1. We only need to introduce three parameters in our proof (the genus of the surface into which we embed, the representativity of the embedding, and the size of the set of vertices to be deleted). On the other hand, Robertson and Seymour consider seven! parameters, see Graph Minor XVI [39]. This leads to a particularly technical and sensitive induction hypothesis in the Robertson-Seymour proof.

2. Our starting point is the "weak structure theorem" as above, while Robertson and Seymour begins with the grid theorem. Our initial setup immediately allows us to focus on a single "special" face and a single "society" because the rest of the graph is essentially embedded into a disc. On the other hand, Robertson and Seymour need to analyze how the rest of the graph attaches to the grid. This requires a lot of work, as in Graph Minors XIV, XV and XVI [37, 38, 39].

3. We only maintain one "special" face and one society at each inductive step, while Robertson and Seymour have to deal with many societies simultaneously. Thus our proof makes the genus addition step much easier. This issue is discussed in Graph Minor XVI [39].

4. In the inductive step, we maintain a subgraph which is essentially embedded in the surface with large representativity. Robertson and Seymour only maintain a subdivision of $G$ embedded in the surface. This difference allows us to deal with the "connectivity" issue more easily. This issue is mainly discussed in Graph Minor XV [38].

5. In addition to a subgraph embedded in the surface, Robertson and Seymour maintain a set of "long" jumps attaching to distant faces of a graph in the surface (see Graph Minor XVI [39]). However, in our case, we simply maintain an embedding on a surface with large representativity.

6. The biggest advantage for our proof is that we do not have to worry about "distance" on a surface. More precisely, Robertson and Seymour have to maintain a subdivision embedded on a surface, but in each inductive step they delete large portions of the subgraph embedded in the surface. In order to maintain the "long" jumps mentioned above, they need to use a technical distance measure for the graph embedded in the surface. This issue is actually quite troublesome in the Graph Minors Series; both Graph Minors XI and XII [34, 35] are devoted to this distance measure. Alternatively, because we do not have to maintain such "long" jumps, we do not rely on this distance measure.

\subsection{Extracting an algorithm}

Our proof is constructive and can be converted into a polynomial time algorithm (in fact $O\left(n^{2}\right)$ time algorithm for fixed $|H|)$ to obtain the structure given in the main structure theorem.

The algorithm follows from our constructive proof. As subroutines, we only need the following.

1. We need to find, for some two vertices $s, t$ and fixed constant $k$, either $k$ disjoint paths between $s$ and $t$, or a vertex set of order at most $k$ that separates $s$ and $t$.

2. We also need a subroutine for 0-near embedding a given society in the disc.

Concerning the first point, we can use the result by Nagamochi and Ibaraki [22] to resolve the problem in $O(n)$ time. Concerning the second point, there is now an $O(n)$ time algorithm to find a 0 -near embedding of a society by Kapadia, Li and Reed [14] (which improves the previous best known result by Tholey [46] which gives $O(m \alpha(m, n))$ time algorithm, where the function $\alpha(m, n)$ is the inverse of the Ackermann function).

At each step of the proof of the main structure theorem, we may proceed by repeatedly calling the above two operations. Therefore, we obtain an $O\left(n^{2}\right)$ time algorithm to find the structure ensured by the main 
structure theorem. For the decomposition theorem, Theorem 1.1, in each iteration, we apply the algorithm of the structure theorem and then recurse on a smaller graph. Thus we obtain an $O\left(n^{3}\right)$ time algorithm to construct the structure in Theorem 1.1. How we extract the $O\left(n^{3}\right)$ time algorithm for the graph minor decomposition from the algorithm for the graph minor structure theorem is treated in more detail in the next section.

\section{Proof of the Decomposition Theorem}

In this section, we prove Theorem 1.1 assuming Theorem 2.1. We recall that a tree decomposition of a graph $G$ is a pair $(T, W)$, where $T$ is a tree and $W$ is a family $\left\{W_{t} \mid t \in V(T)\right\}$ of vertex sets $W_{t} \subseteq V(G)$, such that the following two properties hold:

(W1) $\bigcup_{t \in V(T)} W_{t}=V(G)$, and every edge of $G$ has both ends in some $W_{t}$.

(W2) If $t, t^{\prime}, t^{\prime \prime} \in V(T)$ and $t^{\prime}$ lies on the path in $T$ between $t$ and $t^{\prime \prime}$, then $W_{t} \cap W_{t^{\prime \prime}} \subseteq W_{t^{\prime}}$.

The width of the decomposition is $\min _{t \in V(T)}\left|W_{t}\right|-1$. The treewidth of $G$ is defined as the minimum width taken over all tree decompositions of $G$. For the definition of the torso and a tangle, we refer the reader to Diestel's book [10]. We recall that a separation of a graph $G$ is a pair $(A, B)$ of subsets of vertices such that $G[A] \cup G[B]=G$. We restate the theorem to facilitate the induction.

Theorem 3.1 For every graph $R$ there exist integers $\alpha$ and $\theta$ such that for every graph $G$ that does not contain $R$ as a minor and every $Z \subseteq V(G)$ with $|Z| \leq 3 \theta-2$ there is a rooted tree decomposition $\left\{V_{t} \mid t \in T\right\}$ of $G$ with root $r$ such that for every $t \in V(T)$, there is a surface $\Sigma_{t}$ in which $R$ cannot be embedded, and a subset $A_{t} \subseteq V\left(G_{t}\right)$ of the torso $G_{t}$ of $V_{t}$ with $\left|A_{t}\right| \leq \alpha$ such that $G_{t}-A_{t}$ has an $\alpha$-bounded $\alpha$-near embedding into $\Sigma_{t}$ with the following properties:

(i) There are at most $\alpha$ vortices.

(ii) All vortices have path-width at most $\alpha$.

(iii) For every $t^{\prime} \in V(T)$ with $t t^{\prime} \in E(T)$ and $t \in r T t^{\prime}$ there is a vertex set $X$ which is either

(a) two consecutive parts of a vortex decomposition in $G_{t}$ or

(b) a subset of $V\left(G_{t}\right)$ and induces in $G_{t}$ a $K_{1}$, a $K_{2}$ or a triangle face in $\Sigma_{t}$.

such that $V_{t} \cap V_{t^{\prime}} \subseteq X \cup A_{t}$.

(iv) For every $t^{\prime} \in T$ with $t t^{\prime} \in E(T)$ and $t^{\prime} \in r T t$ the overlap $V_{t} \cap V_{t^{\prime}}$ is contained in $A_{t^{\prime}}$.

Further $Z \subseteq A_{r}$. We say that the part $V_{r}$ accommodates $Z$.

Proof. Applying Theorem 2.1 with the given graph $R$ yields two constants $\hat{\alpha}$ and $\hat{\theta}$. Let $\theta:=\max (\hat{\theta}, 3 \hat{\alpha}+1)$ and $\alpha:=4 \theta-2$.

The proof proceeds by induction on $|V(G)|$. We may assume that $|Z|=3 \theta-2$, since if it is smaller we may arbitrarily add vertices to $Z$. Note, we may assume that such vertices exist, as the theorem is trivial for $|V(G)|<\alpha$.

Claim 3.2 We may assume that there is no separation $(A, B)$ of order at most $\theta$ such that both $|Z \backslash A|$ and $|Z \backslash B|$ are of size at least $|A \cap B|$. 
Proof. Otherwise, let $Z_{A}:=(A \cap Z) \cup(A \cap B)$. By assumption, $|A \cap B| \leq|Z \backslash A|$ and therefore, $\left|Z_{A}\right| \leq|Z|$. We apply our theorem inductively to $G[A]$ and $Z_{A}$, which yields a tree decomposition of $G[A]$ with one part $G_{A}$ such that the apex set of the embedding of its torso contains $Z_{A}$. Similarly, we apply the theorem to $G[B]$ and $Z_{B}:=(B \cap Z) \cup(A \cap B)$. We combine these two tree decompositions by joining a new part $Z \cup(A \cap B)$ to both $G_{A}$ and $G_{B}$ and obtain a tree decomposition of $G$ with the desired properties of the theorem: The new part contains at most $|Z|+|A \cap B| \leq 4 \theta-2$ vertices, so all these can be put into the apex set of an $\alpha$-near embedding. Further, the new part contains $Z$. This proves the claim.

Let $\mathcal{T}$ be the set of separations $(A, B)$ of $G$ of order less than $\theta$ such that $|Z \cap B|>|Z \cap A|$. With this definition, we make the following claim.

Claim $3.3 \mathcal{T}$ is a tangle of $G$ of order $\theta$.

Proof. For every separation $(A, B)$ of $G$ of order less than $\theta$, one of the sets $Z \backslash B$ and $Z \backslash A$ contains at least $\theta$ vertices, as $|Z|=3 \theta-2$, but not both by (3.2). Therefore, property (i) of the definition of a tangle holds. We deduce further, that for every $(A, B) \in \mathcal{T}$, the small side $A$ contains less than $\theta$ vertices from $Z$. Hence, the union of three small sides cannot be $V(G)$ as it contains at most $3 \theta-3$ vertices from $Z$, which shows property (ii) and proves the claim.

From Claim 3.2 and the definition of $\mathcal{T}$ we conclude the following claim.

Claim 3.4 $|(A-B) \cap Z|<|A \cap B|$ for every $(A, B) \in \mathcal{T}$.

Theorem 2.1 implies that there exists a subset $\hat{A} \subseteq V(G)$ with $|\hat{A}| \leq \alpha$ such that there exists an $\hat{\alpha}$-bounded $\hat{\alpha}$-near embedding of $G$ in some surface $\Sigma$ that captures $\mathcal{T}$. At a high level, our plan is now to split up $G$ at separators consisting of apex vertices, society vertices $\Omega\left(H_{i}\right)$ for $i>\hat{\alpha}$ and vertices of single parts of vortex decompositions of a vortex $H_{i}$ for $i \leq \hat{\alpha}$. We obtain a part that contains $H_{0}$ and which we know how to embed $\alpha$-nearly; this part is going to be one part of a new tree decomposition. We find tree decompositions for all subgraphs of $G$ that we split off inductively and eventually combine these tree decompositions to a new one that satisfies our theorem.

Let us consider a small vortex $\left(H_{i}, \Omega_{i}\right)$ for $i \in\{\hat{\alpha}+1, \ldots, m\}$. Our embedding captures $\mathcal{T}$, therefore the separation $\left(V\left(H_{i}\right) \cup \hat{A}, V\left(G-\left(V\left(H_{i}\right)-V\left(H_{0}\right)\right)\right) \cup \hat{A}\right)$, whose order is smaller than $3+|\hat{A}| \leq \theta$, lies in $\mathcal{T}$. By (3.4), $H_{i}$ contains less than $\theta$ vertices of $Z$. Thus, $Z^{\prime}:=\Omega_{i} \cup \hat{A} \cup\left(Z \cap V\left(H_{i}\right)\right)$ contains at most $3+\hat{\alpha}+\theta \leq 3 \theta-1$ vertices. We apply our theorem inductively to the smaller graph $G\left[V\left(H_{i}\right) \cup \hat{A}\right]$ with $Z^{\prime}$. Let $H^{i}$ be a part of the resulting tree decomposition $\left(T^{i}, \mathcal{H}^{i}\right)$ that accommodates $Z^{\prime}$. Let $\mathcal{W}=\left\{H_{\hat{\alpha}+1}, \ldots, H_{m}\right\}$.

For every vortex $\left(H_{i}, \Omega_{i}\right)$ with $\Omega_{i}=\left\{w_{1}^{i}, \ldots, w_{n(i)}^{i}\right\}$ for $i=1, \ldots, \hat{\alpha}$, let us choose a fixed decomposition $\left(\hat{X}_{1}^{i}, \ldots, \hat{X}_{n(i)}^{i}\right)$ of depth at most $\hat{\alpha}$. We define

$$
X_{j}^{i}:= \begin{cases}\left(\hat{X}_{1}^{i} \cap \hat{X}_{2}^{i}\right) \cup\left\{w_{1}^{i}\right\} & \text { for } j=1 \\ \left(\hat{X}_{j}^{i} \cap\left(\hat{X}_{j-1}^{i} \cup \hat{X}_{j+1}^{i}\right)\right) \cup\left\{w_{j}^{i}\right\} & \text { for } 1<j<n(i) \\ \left(\hat{X}_{n(i)}^{i} \cap \hat{X}_{n(i)-1}^{i}\right) \cup\left\{w_{n(i)}^{i}\right\} & \text { for } j=n(i)\end{cases}
$$

By $H_{i}^{-}$we denote the graph on $X_{1}^{i} \cup \ldots \cup X_{n(i)}^{i}$ where every $X_{j}^{i}$ induces a complete graph but no further edges are present. Now, as the depth of $\left(H_{i}, \Omega_{i}\right)$ is at most $\hat{\alpha}$, every $X_{j}^{i}$ contains at most $2 \hat{\alpha}+1$ vertices and thus, $\left(X_{1}^{i}, \ldots, X_{n(i)}^{i}\right)$ is a decomposition of the vortex $V_{i}^{-}:=\left(H_{i}^{-}, \Omega_{i}\right)$ of width at most $2 \hat{\alpha}+1 \leq \alpha$. Let $\mathcal{V}$ denote the set of these new vortices.

For every $j=1, \ldots, n(i)$, the pair

$$
\left(\hat{X}_{j}^{i} \cup \hat{A},\left(V(G)-\left(\hat{X}_{j}^{i}-X_{j}^{i}\right)\right) \cup \hat{A}\right)
$$


is a separation of order at most $\left|X_{j}^{i} \cup \hat{A}\right| \leq 2 \hat{\alpha}+1+\hat{\alpha} \leq \theta$. As before, our embedding captures $\mathcal{T}$ and thus, the separation lies in $\mathcal{T}$. By (3.4), at most $\theta-1$ vertices from $Z$ lie in $\hat{X}_{j}^{i}$. Let $Z^{\prime}:=X_{j}^{i} \cup \hat{A} \cup\left(Z \cap \hat{X}_{j}^{i}\right)$. This set contains at most $3 \theta-1$ vertices and, similar to before, we can apply our theorem inductively to the smaller graph $G\left[\hat{X}_{j}^{i} \cup \hat{A}\right]$ with $Z^{\prime}$. We obtain a tree decomposition $\left(T_{j}^{i}, \mathcal{H}_{j}^{i}\right)$ of this graph, with one part $H_{j}^{i}$ accommodating $Z^{\prime}$.

Now, with $V_{0}:=V\left(H_{0}\right) \cup \hat{A}$, we can write

$$
G=G\left[H_{0}\right] \cup(\bigcup \mathcal{W}) \cup\left(\bigcup\left\{G\left[\hat{X}_{j}^{i}\right]: V_{i} \in \mathcal{V}, 1 \leq j \leq n(i)\right\}\right) .
$$

By induction, we obtained tree decompositions for all vortices in $\mathcal{W}$ and all the graphs $G\left[\hat{X}_{j}^{i}\right]$ with the required properties. We can now construct a tree decomposition of $G$ : We just add a new vertex $v_{0}$ representing $V_{0}$ to the union of all the trees $T^{i}$ and $T_{j}^{i}$ and add edges from $v_{0}$ to every vertex representing an $H^{i}$ or an $H_{j}^{i}$ we found in our proof.

We still have to check that the torso of the new part $V_{0}$ can be $\alpha$-nearly embedded as desired. But this is easy: Let $H_{0}^{\prime}$ be the graph resulting from $H_{0}$ if we add an edge $x y$ for every two nonadjacent vertices $x$ and $y$ that lie in a common vortex $V \in \mathcal{W}$. We can extend the embedding $\sigma: G_{0} \hookrightarrow \Sigma$ to an embedding $\sigma^{\prime}: G_{0}^{\prime} \hookrightarrow \Sigma$ by mapping the new edges disjointly to the $\operatorname{discs} D(V)$. Then, $G^{\prime}:=H_{0}^{\prime} \cup \bigcup H_{i}^{-} \cup \hat{A}$ is the torso of $V_{0}$ in our new tree decomposition and we have an $\alpha$-bounded $\alpha$-near embedding of $G^{\prime}-\hat{A}$ in $\Sigma$.

Observe that the above proof of Theorem 3.1 is constructive and consequently, it gives rise to an $O\left(n^{3}\right)$ time algorithm to construct the decomposition as in Theorem 3.1.

To extract the algorithm, the first step is to define the tangle $\mathcal{T}$ of order $\Theta$. This can be done in $O(n)$ time given that $|Z| \leq 3 \Theta-2$ is an absolute constant and we just need the standard max-flow, minimum-cut algorithm. Then we apply the algorithm to give the structure in Theorem 2.1 with respect to the tangle $\mathcal{T}$, as in the previous section. Finally, we recursively apply this algorithm to all the graphs in $\mathcal{W}$ and all the graphs $G\left[\hat{X}_{j}^{i}\right]$ with the required properties. We can put these decompositions together, as in the proof of Theorem 3.1, to obtain a tree decomposition of $G$ by adding a new vertex $v_{0}$ representing $V_{0}:=V\left(H_{0}\right) \cup \bigcup H_{i}^{-} \cup \hat{A}$ (as in the above proof) to the union of all the trees $T^{i}$ and $T_{j}^{i}$ and add edges from $v_{0}$ to every vertex representing an $H^{i}$ or an $H_{j}^{i}$ we found in our proof.

Since we only need to apply the algorithm to construct the structure in Theorem 2.1 recursively, we obtain an $g(t) n^{3}$ time algorithm to construct the structure as in Theorem 3.1 for some function $g(t)$, as claimed.

\section{References}

[1] N. Alon, P. D. Seymour, and R. Thomas, A separator theorem for non-planar graphs, J. Amer. Math. Soc., 3 (1990), 801-809.

[2] B. S. Baker, Approximation algorithms for NP-complete problems on planar graphs, J. Assoc. Comput. Mach., 41 (1994), 153-180.

[3] H. L. Bodlaender, A linear-time algorithm for finding tree decomposition of small treewidth, SIAM J. Comput., 25 (1996), 1305-1317.

[4] M. Charikar and A. Sahai, Dimension reduction in the $l_{1}$ norm, Proceedings of the 43th Annual Symposium on Foundations of Computer Science (FOCS'02), (2002), 551-560.

[5] C. Chekuri, A. Gupta, I. Newman, Y. Rabinovich, and S. Alistair, Embedding k-outerplanar graphs into $\ell_{1}$, Proceedings of the 14th Annual ACM-SIAM Symposium on Discrete Algorithms (SODA'03), (2003), 527-536. 
[6] E. D. Demaine, F. Fomin, M. Hajiaghayi, and D. Thilikos, Subexponential parameterized algorithms on bounded-genus graphs and $H$-minor-free graphs, J. ACM 52 (2005), 1-29.

[7] E. D. Demaine and M. Hajiaghayi, Fast algorithms for hard graph problems: Bidimensionality, minors and local treewidth, Proc. 12th Internat. Symp. on Graph Drawing, Lecture Notes in Computer Science 3383, Springer, 2004, pp. 517-533.

[8] E. D. Demaine, M. Hajiaghayi, and K. Kawarabayashi, Algorithmic graph minor theory: Decomposition, approximation and coloring, Proc. 46th Annual Symposium on Foundations of Computer Science (FOCS'05), (2005), 637-646.

[9] M. DeVos, G. Ding, B. Oporowski, D. Sanders, B. Reed, P. Seymour, and D. Vertigan, Excluding any graph as a minor allows a low tree-width 2-coloring. J. Combin. Theory Ser. B, 91 (2004), 25-41.

[10] R. Diestel, Graph Theory, 3rd Edition, Springer, 2005.

[11] D. Eppstein, Diameter and treewidth in minor-closed graph families, Algorithmica, 27 (2000), 275-291.

[12] M. Grohe, Local tree-width, excluded minors and approximation algorithms, Combinatorica, 23 (2003), 613-632

[13] A. Gupta, I. Newman, Y. Rabinovich, and S. Alistair, Cuts, trees and $\ell_{1}$-embeddings of graphs, Proc. of the 40th Annual Symposium on Foundations of Computer Science (FOCS'99), (1999), 399-409.

[14] R. Kapadia, Z. Li and B. Reed, A linear time algorithm to test the 2-paths problem, submitted.

[15] K. Kawarabayashi and B. Mohar, Approximating the chromatic number and the list-chromatic number of minor-closed family of graphs and odd-minor-closed family of graphs, Proc. the 38th ACM Symposium on Theory of Computing (STOC'06), (2006), 401-416.

[16] K. Kawarabayashi, S. Norine, R. Thomas, and P. Wollan, $K_{6}$ minors in large 6-connected graphs, submitted.

[17] K. Kawarabayashi and P. Wollan, A shorter proof of the graph minors algorithm - the unique linkage theorem, Proc. 42nd ACM Symposium on Theory of Computing (STOC'10), (2010), 687-694. A full

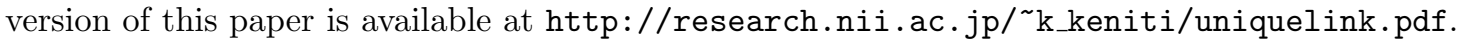

[18] K. Kawarabayashi and B. Reed, A nearly linear time algorithm for the half disjoint paths packing, Proc. 19th Ann. ACM-SIAM Symp. on Discrete Algorithms (SODA'08), (2008), 446-454.

[19] K. Kawarabayashi and B. Reed, A separator theorem in minor-closed classes, Proc. 51st Annual Symposium on Foundations of Computer Science (FOCS'10), (2010), 153-162.

[20] K. Kawarabayashi, Y. Kobayashi and B. Reed, The disjoint paths problem in quaratic time, submitted. Available at http://research.nii.ac.jp/ ${ }^{\sim}$ _keniti/quaddp1.pdf.

[21] P. N. Klein, S. A. Plotkin, and S. Rao, Excluded minors, network decomposition, and multicommodity flow, Proceedings of the 25th Annual ACM Symposium on Theory of Computing (STOC'93), (1993), 682-690.

[22] H. Nagamochi and T. Ibaraki, A linear-time algorithm for finding a sparse $k$-connected spanning subgraph of a $k$-connected graph, Algorithmica 7 (1992), 583-596.

[23] R. J. Lipton, R. E. Tarjan, Applications of a planar separator theorem, SIAM J. Comput., 9 (1980), 615-627.

[24] L. Lovász, Graph minor theory, B. Amer. Math. Soc., 43 (2005), 75-86.

[25] W. Mader, Über die Maximalzahl kreuzungsfreier H-Wege, Archiv der Mathematik (Basel), 31 (1978), $387-402$. 
[26] B. Mohar and C. Thomassen, Graphs on Surfaces, Johns Hopkins University Press, Baltimore, MD, 2001.

[27] S. A. Plotkin, S. Rao, and W. D. Smith, Shallow excluded minors and improved graph decompositions, Proceedings of the 5th Annual ACM-SIAM Symposium on Discrete Algorithms (SODA'94), (1994), $462-470$.

[28] B. Reed, Tree width and tangles: a new connectivity measure and some applications, in Surveys in Combinatorics, London Math. Soc. Lecture Note Ser. 241, Cambridge Univ. Press, Cambridge, 1997, $87-162$.

[29] N. Robertson and P. D. Seymour, Graph minors. III. Planar tree-width, J. Combin. Theory Ser. B, 36 (1984), 49-63.

[30] N. Robertson and P. D. Seymour, Graph minors. VII. Disjoint paths on a surface, J. Combin. Theory Ser. B, 45 (1988), 212-254.

[31] N. Robertson and P. D. Seymour, Graph minors. VIII. A kuratowski theorem for general surfaces, J. Combin. Theory Ser. B, 45 (1988), 212-254.

[32] N. Robertson and P. D. Seymour Graph minors. IX: Disjoint crossed paths, J. Combin. Theory Ser. $B, 49$ (1990), 40-77.

[33] N. Robertson and P. D. Seymour, Graph minors. X: Obstructions to tree decomposition, J. Combin. Theory Ser. B, 52 (1991), 153-190.

[34] N. Robertson and P. D. Seymour, Graph minors. XI. Circuits on a surface, J. Combin. Theory Ser. B, 60 (1994) $72-106$.

[35] N. Robertson and P. D. Seymour, Graph minors. XII. Distance on a surface, J. Combin. Theory Ser. B, 64 (1995) 240-272.

[36] N. Robertson and P. D. Seymour, Graph minors. XIII. The disjoint paths problem, J. Combin. Theory Ser. B, 63 (1995), 65-110.

[37] N. Robertson and P. D. Seymour, Graph minors. IVX. Extending an embedding, J. Combin. Theory Ser. B, 65 (1995), 23-50.

[38] N. Robertson and P. D. Seymour, Graph minors. XV. Giant Steps, J. Combin. Theory Ser. B, 68 (1996), 112-148.

[39] N. Robertson and P. D. Seymour, Graph minors. XVI: Excluding a non-planar graph, J. Combin. Theory Ser. B, 89 (2003), 43-76.

[40] N. Robertson and P. D. Seymour, Graph minors. XVII: Taming a Vortex, J. Combin. Theory Ser. B, 77 (1999), 162-210.

[41] N. Robertson and P. D. Seymour, Graph minors. XX. Wagner's conjecture, J. Combin. Theory Ser. B, 92 (2004), 325-357.

[42] N. Robertson and P. D. Seymour, Graph minors. XXI: Graphs with unique linkages, J. Combin. Theory Ser. B, 99 (2009), 583-616.

[43] N. Robertson, P. D. Seymour and R. Thomas, Quickly excluding a planar graph, J. Combin. Theory Ser. B, 62 (1994), 323-348.

[44] A. Schrijver, A Short Proof of Mader's S-path Theorem, J. Combin. Theory Ser. B, 82 (2001), 319-321.

[45] P. D. Seymour, Disjoint paths in graphs, Discrete Mathematics, 29 (1980), 293 - 309.

[46] T. Tholey, Solving the 2-disjoint paths problem in nearly linear time, Theory of computing systems 39 (2004), 51-78. 
[47] C. Thomassen, 2-linked graph, European Journal of Combinatorics, 1 (1980), 371 - 378.

[48] K. Wagner, Über eine Eigenschaft der ebenen Komplexe, Math. Ann., 114 (1937), 570-590.

\section{Appendix}

The appendix is organized as follows. In section A, we give notation used in this paper. Sections B and C are useful lemmas for our society theorem. Theorem 2.2 is proven in Section D, and Theorem 2.3 will be shown in Section E. Finally in Section 7, we give our main proof the main structure theorem.

\section{A Notation}

In this section, we begin with the notation which will be necessary to state the society theorem strengthening the main result of [32].

We mention several definitions on systems paths in graphs which we will use throughout the article. First, a linkage $\mathcal{P}$ is a graph such that each component of $\mathcal{P}$ is a path. The order of a linkage is the number of components. We will use $P \in \mathcal{P}$ to indicate that $P$ is a component of $\mathcal{P}$. We allow that some components of $\mathcal{P}$ be equal to trivial paths on a single vertex. A sub-linkage of $\mathcal{P}$ is any subgraph of $\mathcal{P}$ which is itself a linkage.

In a graph $G$, for any set $X$ of vertices in $G$, an $X$-path is a path $P$ in $G$ such that both endpoints of $P$ are contained in $X$ and $P$ has no internal vertex in $X$.

\section{A.1 Societies and patterns}

A society is a pair $(G, \Omega)$ where $G$ is a graph and $\Omega$ is a cyclic ordering of a subset of the vertices of $G$. In somewhat sloppy notation, we will use $\Omega$ to refer both to the cyclic order as well as the set of vertices itself. A segment of a society is a non-empty, proper subset $X \subseteq \Omega$ such that there do not exist $x_{1}, x_{2} \in X$ and $y_{1}, y_{2} \in \Omega \backslash X$ such that $x_{1}, y_{1}, x_{2}, y_{2}$ occur on $\Omega$ in that order. A segment can thus be thought of as a continuous section of the vertices in $\Omega$. Thus for any two vertices $u, v$ in $G$, we can define the segments $u \Omega v$ and $v \Omega u$ such that the intersections of the two segments are only $u$ and $v$.

Given two disjoint paths $\Omega$-paths $P_{1}$ and $P_{2}$, we say that $P_{1}$ and $P_{2}$ are crossing if we can label the endpoints of $P_{i}$ as $s_{i}$ and $t_{i}$ for $i=1,2$ such that $s_{1}, s_{2}, t_{1}, t_{2}$ occur in $\Omega$ in that order. An $\Omega$-bridge in $G$ is a connected subgraph $B$ of $G$ such that either $E(B)$ consists of a unique edge with both endpoints in $\Omega$, or for some component $C$ of $G-\Omega$, the set $E(B)$ consists of all edges of $G$ with at least one endpoint in $V(C)$.

A pattern in a society $(G, \Omega)$ is a linkage where each component is an $\Omega$-path. In very brief terms, we will show that either a society contains one of several desirable patterns, or, alternatively, admits a decomposition. We first define the possible patterns we will consider.

The most simple is a $k$-crosscap. For a positive integer $k, \mathrm{~A} k$-crosscap in a society $(G, \Omega)$ is a pattern $\mathcal{P}$ with $|\mathcal{P}|=k$ such that the elements of $\mathcal{P}$ are pairwise crossing.

Definition Let $(G, \Omega)$ be a society and $k \geq 1, l \geq 1$ positive integers. A $(k, l)$-cross pattern in $(G, \Omega)$ consists of the union of three linkages $\mathcal{P}, \mathcal{Q}$, and $\mathcal{R}$ of $\Omega$-paths satisfying the following conditions.

i. The components of $\mathcal{P}, \mathcal{Q}$, and $\mathcal{R}$ are pairwise vertex-disjoint.

ii. We can label the components of $\mathcal{Q}$ as $Q_{1}, \ldots, Q_{k}$ and the components of $\mathcal{R}$ as $R_{1}, \ldots, R_{k}$, so that the paths $Q_{i}$ and $R_{i}$ are crossing for all $1 \leq i \leq k$. 
iii. For all $P, P^{\prime} \in \mathcal{P}, P$ and $P^{\prime}$ do not cross. It follows that $\Omega \cup \mathcal{P}$ can be embedded in the disc with the vertices of $\Omega$ on the boundary of the disc in the indicated order.

iv. Fix such an embedding of $\mathcal{P} \cup \Omega$ in the disc. For every $1 \leq i \leq l$, there exists a face $F_{i}$ (not infinite face that corresponds to $\Omega$ ) containing all the endpoints of $Q_{i} \cup R_{i}$, and in the dual graph of the embedding of $\mathcal{P} \cup \Omega$ (minus the vertex that corresponds to the infinite face that corresponds to $\Omega$ ), the faces $F_{i}$ and $F_{j}$ have distance at least $l$ for all $1 \leq i<j \leq l$.

Moreover, we assume that $\mathcal{P}$ is inclusion minimal satisfying $i-i v$. In other words, if we delete some component of $\mathcal{P}$, we no longer satisfy $i v$.

Note that a $(1, l)$-cross pattern consists simply of the pair of crossing paths $R_{1}$ and $R_{2}$. An $l$-doublecross, as defined by Robertson and Seymour in [32], is the same as a $(2, l)$-cross pattern.

Let $\mathcal{P}$ be a pattern with pairwise non-crossing components in a society $(G, \Omega)$. Then there exists a natural embedding of $\mathcal{P} \cup \Omega$ in the disc such that the vertices contained in the boundary of the disc are exactly the vertices of $\Omega$ in the stipulated cyclic order. For any two vertices $x$ and $y$ in $\Omega \cup V(\mathcal{P})$, we say the distance between $x$ and $y$ is the minimal number of times a curve $C$ in the disc with endpoints $x$ and $y$ intersects $\mathcal{P}$. Since the embedding of $\mathcal{P} \cup \Omega$ in the disc is unique up to homeomorphism of the disc, this distance function is a property only of the pattern $\mathcal{P}$ and not the embedding. Thus, we denote this distance function $d_{\mathcal{P}}$. Note that it is easy to see that $d_{\mathcal{P}}$ satisfies the triangle inequality, although it will not generally define a metric.

Definition Let $k, l$, be non-negative integers. Let $(G, \Omega)$ be a society. A $(k, l)$-leap pattern is a pattern $\mathcal{P} \cup \mathcal{Q}$ satisfying the following conditions.

i. The components of $\mathcal{P}$ and $\mathcal{Q}$ are pairwise disjoint.

ii. For all $P, P^{\prime} \in \mathcal{P}, P$ and $P^{\prime}$ do not cross.

iii. The pattern $\mathcal{Q}$ has order $k$, and for all $Q \in \mathcal{Q}$ the endpoints of $Q$ have distance $d_{\mathcal{P}}$ at least $l$.

Moreover, we assume that $\mathcal{P}$ is inclusion minimal satisfying $i$-iii, i.e. after deleting a component of $\mathcal{P}$, the resulting pattern does not satisfy iii.

Observation 1 For all $k \geq 1, l \geq 1$, both a $(k, l)$-cross pattern and $a(k, l)$-leap pattern have at most $\left(\begin{array}{c}k \\ 2\end{array}\right) l+2 k$ components.

A special type of $(k, k)$-leap pattern is an $k$-handle.

Definition A $k$-handle is a leap pattern formed by the union $\mathcal{P} \cup \mathcal{Q}$ of two linkages of order $k$ satisfying the following conditions.

i. The components of $\mathcal{P}$ and $\mathcal{Q}$ are pairwise disjoint.

ii. For every $P, P^{\prime} \in \mathcal{P}, P$ and $P^{\prime}$ do not cross. Similarly, for each $Q, Q^{\prime} \in \mathcal{Q}, Q$ and $Q^{\prime}$ do not cross.

iii. For all $P \in \mathcal{P}$ and $Q \in \mathcal{Q}, P$ and $Q$ cross.

\section{A.2 Tangles and Grids}

A (directed) separation of a graph $G$ is an ordered pair $(A, B)$ of non-empty subsets of $V(G)$ such that $G[A] \cup G[B]=G$. The number $|A \cap B|$ is the order of $(A, B)$. Whenever we speak of separations in this paper, we shall mean such directed separations. 
Definition A set $\mathcal{T}$ of separations of $G$, all of order less than some integer $\theta$, is a tangle of order $\theta$ if the following holds:

i. For every separation $(A, B)$ of $G$ of order less than $\theta$, either $(A, B)$ or $(B, A)$ lies in $\mathcal{T}$.

ii. If $\left(A_{i}, B_{i}\right) \in \mathcal{T}$ for $i=1,2,3$, then $G\left[A_{1}\right] \cup G\left[A_{2}\right] \cup G\left[A_{3}\right] \neq G$.

Note that if $(A, B) \in \mathcal{T}$ then $(B, A) \notin \mathcal{T}$; we think of $A$ as the 'small side' of the separation $(A, B)$, with respect to this tangle. We also call $B$ as a 'big side', with respect to this tangle.

For a tangle $\mathcal{T}$ of order $\theta$ in a graph $G$, let $Z \subseteq V(G)$ be a vertex set with $|Z|<\theta$. Let $\mathcal{T}-Z$ denote the set of all separations $\left(A^{\prime}, B^{\prime}\right)$ of $G-Z$ of order less than $\theta-|Z|$ such that there exists a separation $(A, B) \in \mathcal{T}$ with $Z \subseteq A \cap B, A-Z=A^{\prime}$ and $B-Z=B^{\prime}$. It is shown in [33, Theorem (6.2)] that $\mathcal{T}-Z$ is a tangle of order $\theta-|Z|$ in $G-Z$. Thus we can also call $B$ as a 'big side', with respect to this tangle.

For positive even integers $r$, define a graph $H_{r}$ as follows. Let $P_{0}, \ldots, P_{r}$ be $r$ vertex disjoint ('horizontal') paths of length $2 r+1$, say $P_{i}=v_{0}^{i} \ldots v_{2 r+1}^{i}$. Let $V\left(H_{r}\right)=\bigcup_{i=1}^{r} V\left(P_{i}\right) \backslash\left\{v_{0}^{0}, v_{2 r+1}^{r}\right\}$, and let

$$
\begin{array}{r}
E\left(H_{r}\right)=\left(\bigcup_{i=1}^{r} E\left(P_{i}\right) \backslash\left\{v_{0}^{0} v_{1}^{0}, v_{2 r}^{r} v_{2 r+1}^{r}\right\}\right) \cup\left\{v_{j}^{i} v_{j}^{i+1}: i \text { odd, } j \text { even; } 1 \leq i<r ; 0 \leq j \leq 2 r+1\right\} \\
\cup\left\{v_{j}^{i} v_{j}^{i+1}: i \text { even, } j \text { odd } ; 0 \leq i<r ; 1 \leq j \leq 2 r+1\right\} .
\end{array}
$$

The 6-cycles in $H_{r}$ are its bricks. In the natural plane embedding of $H_{r}$, these bound its 'finite' faces. The outer cycle of the unique maximal 2-connected subgraph of $H_{r}$ is the boundary cycle of $H_{r}$. Any subdivision $H=T H_{r}$ of $H_{r}$ will be called an $r$-wall or a wall of size $r$. The bricks and the boundary cycle of $H$ are its subgraphs that form subdivisions of the bricks and the boundary cycle of $H_{r}$, respectively. The nails of $H$ are vertices of degree 3 in $H$.

Let us recall that an $r$-grid is a graph which is isomorphic to a subdivision of the graph $W_{r}$ obtained from the Cartesian product of paths $P_{r} \times P_{r}$, with vertex set $V\left(W_{r}\right)=\{(i, j) \mid 1 \leq i \leq r, 1 \leq j \leq r\}$ in which two vertices $(i, j)$ and $\left(i^{\prime}, j^{\prime}\right)$ are adjacent if and only if $\left|i-i^{\prime}\right|+\left|j-j^{\prime}\right|=1$. The $(a \times b)$-grid is defined in a similar way.

Definition A tree decomposition of a graph $G$ is a pair $(T, W)$, where $T$ is a tree and $W$ is a family $\left\{W_{t} \mid t \in V(T)\right\}$ of vertex sets $W_{t} \subseteq V(G)$, such that the following two properties hold:

(W1) $\bigcup_{t \in V(T)} W_{t}=V(G)$, and every edge of $G$ has both ends in some $W_{t}$.

(W2) If $t, t^{\prime}, t^{\prime \prime} \in V(T)$ and $t^{\prime}$ lies on the path in $T$ between $t$ and $t^{\prime \prime}$, then $W_{t} \cap W_{t^{\prime \prime}} \subseteq W_{t^{\prime}}$.

The width of the decomposition is $\min _{t \in V(T)}\left|W_{t}\right|-1$. The treewidth of $G$ is defined as the minimum width taken over all tree decompositions of $G$.

A path decomposition is a tree decomposition where the underlying graph is a path. We will often indicate path decompositions simply by a linearly ordered collection $\mathcal{B}_{i}, 1 \leq i \leq k$, of subsets $V(G)$ where the path is understood to be the path with vertex set $\{i \mid 1 \leq i \leq k\}$ and edges $\{i(i+1) \mid 1 \leq i \leq k-1\}$.

One of the most important results concerning the treewidth is that it guarantees the existence of a large wall. We give an algorithmic version of this result, which is due to Bodlaender [3].

Theorem A.1 For any constant $r$, there exists a constant $w=f_{1}(r)$ satisfying the following: There exists an $O\left(w^{w} n\right)$ time algorithm that, given a graph $G$, either finds a tree decomposition of $G$ of width at most $w$ or finds a wall $W$ of height $r$. For a planar graph, the time complexity can be improved to $O\left(2^{w} n\right)$. 
Let $\mathcal{T}$ be a tangle of order $\Theta$. We say that a wall of height at least $\Theta$ is controlled by the tangle $\mathcal{T}$ if the 'large side' $B$ of an element $(A, B) \in \mathcal{T}$ contains all but at most $\Theta$ nails of $H$. There is a corresponding result of Theorem A.1 that is concerning about a big side of a given tangle of large order.

Theorem A.2 For any constant $r$, there exists a constant $w=f_{2}(r)$ satisfying the following: Let $\mathcal{T}$ be a tangle of order $w$. Then there is a wall of height $r$, controlled by the tangle $\mathcal{T}$. Furthermore, given the tangle $\mathcal{T}$, there exists an $O\left(w^{w} n\right)$ time algorithm that finds a wall $W$ of height $r$.

The first half of Theorem A.2 was proved by Robertson, Seymour and Thomas [43], while the second half is obtained by Reed, see [18, 28].

\section{A.3 Flat decompositions}

We now describe the decomposition when a given society does not contain any of the desired patterns.

Vortex decompositions are a similar idea of that of tree decompositions, extended to societies. Let $(G, \Omega)$ be a society, and label the vertices of $\Omega v_{1}, v_{2}, \ldots, v_{n}$ for some positive integer $n$ in the order in which they occur in $\Omega$.

Definition A vortex decomposition of $(G, \Omega)$ is a collection of subsets $X_{1}, \ldots, X_{n} \subseteq V(G)$ such that

i. for all $1 \leq i \leq n, v_{i} \in X_{i}$ and for every edge $e \in G$, there exists an index $j$ such that both ends of $e$ are contained in $X_{j}$.

ii. for all $v \in V(G),\left\{v_{j} \mid v \in X_{j}\right\}$ forms a segment of $(G, \Omega)$.

The depth of a vortex decomposition is equal to the

$$
\max _{i, j}\left|X_{i} \cap X_{j}\right|
$$

Just as large treewidth ensures the existence of a large grid minor, we see that if a society does not admit a vortex decomposition of small depth, then it must contain a certain structure.

Theorem A.3 (8.1, [32]) For all $t \geq 1$, if $(G, \Omega)$ has no vortex decomposition of depth at most $t$ then there exist two disjoint segments $S_{1}, S_{2}$ of $\Omega$ and $t$ disjoint $\Omega$-paths each with one endpoint in $S_{1}$ and one endpoint in $S_{2}$.

Definition Let $(G, \Omega)$ be a society and $t \geq 0$ and $\alpha \geq 1$ be integers. Then $(G, \Omega)$ admits a $t$-nearly flat decomposition if the following hold. There exists an integer $n \geq t$ and pairwise edge disjoint subgraphs $H_{0}, H_{1}, \ldots, H_{t}, H_{t+1}, \ldots H_{n}$ of $G$ satisfying the following:

i. $\bigcup_{i=0}^{n} H_{i}=G$ and $\Omega \subseteq V\left(H_{0}\right)$.

ii. For all $i, j \geq 1, i \neq j, V\left(H_{i}\right) \cap V\left(H_{j}\right) \subseteq V\left(H_{0}\right)$.

iii. For all $i>t,\left|V\left(H_{i}\right) \cap V\left(H_{0}\right)\right| \leq 3$.

iv. Let $\Sigma$ be the disc. There exist pairwise disjoint open discs $\Delta_{1}, \ldots, \Delta_{n}$ and an embedding $\sigma: H_{0} \hookrightarrow$ $\Sigma-\bigcup_{i=1}^{n} \Delta_{i}$ satisfying the following.

a. The vertices of $\Omega$ are on the boundary of $\Sigma$ in the order stipulated by $\Omega$.

b. The only vertices contained in the boundary of $\Delta_{i}$ are exactly the vertices of $H_{i} \cap H_{0}$. 
For each $i, 1 \leq i \leq n$, let $\Omega_{i}$ be the cyclic ordering of the vertices $V\left(H_{0}\right) \cap V\left(H_{i}\right)$ given by the embedding of $H_{0}$ in the plane. The societies $\left(H_{i}, \Omega_{i}\right)$ are the vortices of the decomposition; we refer to $\left(H_{i}, \Omega_{i}\right)$ for $t<i \leq n$ as the small vortices and for $1 \leq i \leq t$ as the large vortices. The decomposition is $\alpha$-bounded if for all $1 \leq i \leq t,\left(H_{i}, \Omega_{i}\right)$ has a vortex decomposition of depth at most $\alpha$. Finally, let $\bar{H}_{0}$ be the graph defined to have vertex set $V\left(H_{0}\right)$ and edge set $E\left(H_{0}\right) \cup\left\{x y \mid \exists i>t\right.$ such that $\left.x, y \in V\left(H_{i}\right) \cap V\left(H_{0}\right)\right\}$. Note that by construction, $\bar{H}_{0}$ embeds in $\Sigma-\bigcup_{i=1}^{r} \Delta_{i}$.

We sometimes say that $(G, \Omega)$ is rural if it admits a 0-nearly flat decomposition. Let $t$ be a positive integer $t$, and let $H_{0}, H_{1}, \ldots, H_{n}$ be a $t$-nearly flat decomposition of a society $(G, \Omega)$. We say that the small vortices of the decomposition are grounded if for all $i, t<i \leq n$, there exists a component $C$ of $H_{i}-V\left(H_{0}\right)$ such that for all $x \in V\left(H_{i}\right) \cap V\left(H_{0}\right)$ there exists a $y \in V(C)$ such that $x$ is adjacent to $y$. In other words, the component $C$ is adjacent every vertex of $V\left(H_{i}\right) \cap V\left(H_{0}\right)$. Thus "grounded" serves as a sort of non-triviality condition for the decomposition.

Observation 2 If a society $(G, \Omega)$ admits a t-nearly flat decomposition for some non-negative integer $t$, then $(G, \Omega)$ admits a t-nearly flat decomposition where the small vortices are grounded.

The $t$-nearly flat decomposition generalizes the idea of almost 3 -embeddings in the disc where we now allow at most $t$ possible faces to be replaced by sub-societies. The crossing paths theorem of Robertson and Seymour can be restated as follows.

Theorem A.4 (2.4, [32]) Let $(G, \Omega)$ be a society. Then $(G, \Omega)$ either contains a $(1, l)$-cross pattern for every positive $l$, or $(G, \Omega)$ admits a 0 -nearly flat embedding.

The next theorem is the main result of [32].

Theorem A.5 (6.4, [32]) For all positive $l$, there exists a value $\alpha=\alpha(l)$ such that the following holds. Let $(G, \Omega)$ be a society. Then $(G, \Omega)$ either

1. contains an l-crosscap, a $(1, l)$-leap pattern, or a $(2, l)$-cross pattern, or

2. admits an $\alpha$-bounded 1-nearly flat decomposition.

We generalize this result and show the following theorem. This is a more exact statement of Theorem 2.2, which follows immediately from the stronger statement.

Theorem A.6 For all non-negative integers $k, l$, there exists a value $\alpha=\alpha(k, l)$ satisfying the following. Let $(G, \Omega)$ be a society. Either

1. $(G, \Omega)$ contains a $k$-crosscap, a $(k, l)$-cross pattern, or a $(k, l)$-leap pattern, or

2. there exists a set $X \subseteq V(G)$ with $|X| \leq \alpha$ such that $(G-X, \Omega \backslash X)$ admits an $\alpha$-bounded $(k-1)$-nearly flat decomposition.

\section{A.4 The weak structure theorem}

In this subsection, we shall mention one of the key theorems we will use, which we call The Weak Structure Theorem. We need some definitions.

Definition For a positive integer $r$ and a graph $G$, a flat $r$-wall decomposition of $G$ is a collection of pairwise edge disjoint subgraphs $G_{0}, G_{1}, \ldots, G_{n}$ and an $r$-wall subgraph $W$ with boundary cycle $C$ satisfying the following: 
i. $G=\bigcup_{i=0}^{n} G_{i}$,

ii. $V\left(G_{i}\right) \cap V\left(G_{j}\right) \subseteq V\left(G_{0}\right)$ for all $1 \leq i<j \leq n$ and $\left|V\left(G_{i}\right) \cap V\left(G_{0}\right)\right| \leq 3$ for all $1 \leq i \leq n$, and

iii. for all $1 \leq i \leq n, V\left(G_{i}\right) \backslash V\left(G_{0}\right)$ contains at most one vertex of degree 3 in $W$.

Let $\overline{G_{0}}$ be the subgraph resulting from $G_{0}$ after adding an edge to any two nonadjacent vertices $u$ and $v$ contained in $V\left(G_{0}\right) \cap V\left(G_{i}\right)$ for some index $1 \leq i \leq n$.

iv. The graph $\overline{G_{0}}$ is planar and can be embedded such that the infinite face is bounded by $V(C) \cap V\left(G_{0}\right)$.

Recall that $\partial_{G}(X)$ for any subset $X$ in a graph $G$ is the set of vertices $v$ in $V(G) \backslash X$ such that $v$ has a neighbor in $X$.

We are now ready to give the Weak Structure Theorem.

Theorem A.7 (Weak Structure Theorem, [36], Theorem (9.4)) For all $k \geq 1, r$ even, there exists a value $q=q(t, r)$ such that the following holds. Let $G$ be a graph that does not contain $K_{t}$ as a minor and let $W$ be a wall of size $w$. Then there exists a set $A \subseteq V(G)$ with $|A| \leq 10 t^{2}$ such that $G-A$ can be decomposed into edge disjoint subgraphs $H$ and $G^{\prime}$ with $G-A=G^{\prime} \cup H$. Moreover, the graph $H$ has a flat $r$-wall decomposition $H_{0}, H_{1}, \ldots, H_{n}$, a subwall $W^{\prime}$ of size $r$ of $W$ with boundary cycle $C$ satisfying:

i) $\partial_{G-A}\left(V\left(G^{\prime}\right)\right) \subseteq V(C) \cap V\left(H_{0}\right)$, and

ii) every vertex of degree 3 in $W^{\prime}$ is contained in $V\left(H_{0}\right)$.

The weak structure theorem is in fact weaker than the full structure theorem. Notice that a graph $G$ may have the desired decomposition in the statement of the weaker theorem, and yet still have an arbitrarily large clique minor (for example, an arbitrarily large clique could be contained in the subgraph $G^{\prime}$ ). Instead, in the full clique-sum decomposition theorem, for all integers $t$ there exists a value $T$ such that any graph with no $K_{t}$ minor has a certain structure, and, moreover, any graph which does have this structure cannot contain $K_{T}$ as a minor. See [10].

Let us observe that if we are given a tangle $\mathcal{T}$ of order $f_{2}(q)$, we can find a $q$-wall $W$ that can be obtained from Theorem A.2, such that $W$ is controlled by the tangle $\mathcal{T}$. If we start with this wall $W$ in Theorem A.7, each $H_{i} \cup A$ is a small side with respect to the tangle $\mathcal{T}$. This fact will be used when we prove Theorem 1.1.

\section{A.5 Near embeddings and the structure theorem}

Before proving the main structure theorem, Robertson and Seymour first studied graphs embedded in some fixed surface. We will use these tools as well, and we present them in this section.

We begin with several basics on graphs embedded in surfaces. By surface we mean a 2-manifold without boundary, and we always assume the embedded graph has a 2-cell embedding. If a graph $G$ is embedded in a surface $\Sigma$ not equal to the sphere, the representativity of the embedding is the minimal number of points in which a homotopically non-trivial curve $C$ in the surface intersects the embedded graph.

We will need the following result or Robertson and Seymour.

Theorem A.8 (9.1,[30]) For all $t \geq 1$ and for all surfaces $\Sigma$ not equal to the sphere in which $K_{t}$ can be embedded, there exists a value $f^{\prime}(t, \Sigma)$ such that if $G$ is a graph embedded in $\Sigma$ with representativity $f^{\prime}(t, \Sigma)$, then $G$ contains $K_{t}$ as a minor.

A $k$-near embedding in $\Sigma$ captures a tangle $\mathcal{T}$ if the 'large side' $B$ of an element $(A, B) \in \mathcal{T}-Z$ is never contained in a $H_{i}$ for $1 \leq i \leq m$. 


\section{B Lemmas on $X$-paths}

Given a fixed subset $X \subseteq V(G)$ of a graph $G$, we define a distance function to be a function $d: X \times X \rightarrow[0, \infty)$ such that

1. $d(x, y)=d(y, x)$ for all $x, y \in X$, and

2. $d(x, y)+d(y, z) \geq d(x, z)$ for all $x, y, z \in X$.

In other words, a distance function is similar to a metric in that it is a symmetric non-negative function which satisfies the triangle inequality. However, we do not include any requirements on when $d(x, y)$ is equal to zero. Given a distance function $d, x \in X$, and $t \geq 0$, we define the ball around $x$ of radius $t$, denoted $B_{d}(x, t)$ or $B(x, t)$ when there can be no confusion, to be $\{y \in X \mid d(x, y) \leq t\}$.

In this section we prove two lemmas on $X$-paths in graphs. Recall that if $X \subseteq V(G)$ is a subset of the vertices of a graph $G$, then an $X$-path is a path $P$ with both endpoints contained in $X$ and no internal vertex in $X$.

Lemma B.1 Let $d$ be a distance function on a subset $X \subseteq V(G)$ of vertices of a graph $G$. Let $k, l, t \geq 0$ be non-negative integers. Then one of the following holds. Either there exist pairwise disjoint $X$-paths $P_{1}, \ldots, P_{k}$ that satisfy the following conditions:

i. $d\left(x_{i}, y_{i}\right) \geq l$ for all $1 \leq i \leq k$, and

ii. the endpoints of $P_{i}$ can be labeled $x_{i}, y_{i}$ so that $d\left(x_{i}, x_{j}\right) \geq t$ for all $1 \leq i<j \leq k$.

Or, alternatively, the following holds. Let $T=\max \{l, t\}$. There exist vertices $z_{1}, \ldots, z_{3 k-3} \in X$ and $Z \subseteq V(G),|Z| \leq k-1$ such that every $X \backslash Z$-path $P$ in $G-Z$ with endpoints $x$ and $y$ either satisfies $d(x, y)<l$ or $x, y \in \bigcup_{i=1}^{3 k-3} B\left(z_{i}, T\right)$.

This is an idea we will return to again later in the article. We refer to a set of $X$-paths satisfying $i$. and $i$. above as $(l, t)$-spread with respect to the distance function $d$.

To summarize, call an $X$-path with endpoints at distance $l$ long. The lemma states that one of the following two situations holds. First, we find $k$ vertex disjoint long $X$-paths $P_{1}, \ldots, P_{k}$ such that the endpoints of $P_{i}$ can be labeled $x_{i}, y_{i}$, so that for each $P_{j}$ and $P_{j^{\prime}}, x_{j}$ is of distance $t$ from $x_{j^{\prime}}$ for all $j \neq j^{\prime}$. Alternatively, we find a small number of vertices $Z$ in $G$ and at most $3 k-3$ balls in $X$ such that every long $X$-path either contains a vertex of $Z$, or has both endpoints contained in the union of these balls.

Proof. Let $k, l$, and $t$ be non-negative integers. Fix $T=\max \{l, t\}$. For the duration of the proof, we will say that an $X$-path $P$ is long if the endpoints of $P$ are at distance at least $l$. We proceed by induction on $|X|$.

First, assume that there exists a vertex $x \in X$ such that there does not exist a long $X$-path with $x$ as an endpoint. Then the theorem holds by simply applying induction to $G-x$. We conclude that every vertex of $X$ is the endpoint of some long $X$-path.

Let $P_{1}, \ldots, P_{s}$ be disjoint $X$-paths satisfying $i$ and $i i$ with $s$ chosen maximal over all such possibilities. Let $0 \leq p \leq s$ be an integer, and let $Q_{1}, \ldots, Q_{p}$ be disjoint paths with the endpoints of $Q_{i}$ equal to $z_{i}$ and $w_{i}$ for $1 \leq i \leq p$ satisfying the following.

a. $w_{i} \in X \backslash \bigcup_{j=1}^{s}\left(B\left(x_{j}, T\right) \cup B\left(y_{j}, T\right)\right)$ for all $1 \leq i \leq p$.

b. $z_{i} \in V\left(P_{i}\right)$ for all $1 \leq i \leq p$. 
c. $Q_{i}$ is internally disjoint from $X \cup \bigcup_{j=1}^{s} V\left(P_{j}\right)$ for $1 \leq i \leq p$.

d. $d\left(w_{i}, w_{j}\right)>T$ for $1 \leq i<j \leq p$.

We pick $P_{1}, \ldots, P_{s}$ and $Q_{1}, \ldots, Q_{p}$ satisfying a - d to maximize $p$. Fix $Z=\left\{z_{1}, \ldots, z_{p}\right\}$

Let $x$ be a vertex of $X \backslash\left(\bigcup_{i=1}^{p} B\left(w_{i}, T\right) \cup \bigcup_{i=1}^{s}\left(B\left(x_{i}, T\right) \cup B\left(y_{i}, T\right)\right)\right)$, and let $R$ be a long $X \backslash Z$-path in $G-Z$. If no such $x$ and $R$ exist, then given that $s \leq k-1$ and $p \leq s$, the lemma is proven. If $R$ is disjoint from $P_{1}, \ldots, P_{s}$, we see that $R, P_{1}, \ldots, P_{s}$ satisfy $i$ and $i i$, contrary to our choice of $P_{1}, \ldots, P_{s}$. Note that $i$ follows by our choice of $x$ to be distant $T$ from every endpoint of the $P_{i}$. We conclude that $V(R) \cap\left(\bigcup_{i=1}^{p} V\left(Q_{i}\right) \cup \bigcup_{i=1}^{s} V\left(P_{i}\right)\right)$ is nonempty. Let $y$ be the first vertex of $\bigcup_{1}^{p} V\left(Q_{i}\right) \cup \bigcup_{1}^{s} V\left(P_{i}\right)$ which we encounter when traversing the path $R$ beginning at the endpoint $x$.

There are now several different cases, depending on where the vertex $y$ lies. As the first case, assume $y \in V\left(Q_{i}\right)$ for some $1 \leq i \leq p$. It follows that $R \cup Q_{i}$ contains a long $X$-path, call it $P^{\prime}$, which has $x$ as an endpoint and is disjoint from $P_{1}, \ldots, P_{s}$. However, then $P^{\prime}, P_{1}, \ldots, P_{s}$ satisfy $i$ and $i i$, violating our choice of $s$ to be maximal. As a case, assume $y \in P_{i}$ for some $1 \leq i \leq p$. Then $R \cup Q_{i} \cup P_{i}$ contains two disjoint long $X$-paths, call them $P^{\prime}$ and $P^{\prime \prime}$, such that $P^{\prime}$ has $x$ as an endpoint and $P^{\prime \prime}$ has $w_{i}$ as an endpoint. Note that here we are using the property that $y \neq z_{i}$ to ensure that $P^{\prime}$ and $P^{\prime \prime}$ can be chosen disjoint. Then $P_{1}, \ldots, P_{i-1}, P_{i+1}, \ldots, P_{s}, P^{\prime}, P^{\prime \prime}$ satisfy $i$ and $i i$, violating our choice of $s$ to be maximal. As the final case, consider when $y \in P_{i}$ for $p<i \leq s$. Then $Q_{1}, \ldots, Q_{p}, R$, after possibly renumbering the paths $P_{1}, \ldots, P_{s}$, satisfy a - d, contrary to our choice of $p$. This completes the analysis of the possible cases and completes the proof of the lemma.

Before proceeding with the next result, we mention a theorem of Mader [25] which we will use in the proof. Let $\mathcal{S}$ be a partition of a subset $X$ of vertices of a graph $G$. An $\mathcal{S}$-path is an $X$-path with endpoints in distinct elements of $\mathcal{S}$. See [44] for a short proof of Theorem B.2.

Theorem B.2 (Mader) Let $G$ be a graph and $\mathcal{S}$ a partition of a subset $S$ of vertices in $G$. The maximum number of disjoint $\mathcal{S}$-paths is equal to the minimum value of

$$
\left|U_{0}\right|+\sum_{i=1}^{n}\left\lfloor\frac{1}{2}\left|B_{i}\right|\right\rfloor
$$

taken over all partitions $U_{0}, U_{1}, \ldots, U_{n}$ of $V(G)$ such that each $\mathcal{S}$-path disjoint from $U_{0}$ has at least one edge with both endpoints in $U_{i}$ for some index $i$. The value $B_{i}$ denotes the set of vertices in $U_{i}$ which belong to $S$ or have at least one neighbor in $V(G) \backslash\left(U_{0} \cup U_{i}\right)$.

The next lemma is somewhat technical and concerns the following situation. We begin with a collection of $k-1$ disjoint $X$-paths each of whose endpoints are distant in $X$, and would like to expand this to a set of $k$ disjoint $X$-paths, again, each of whose endpoints are distant in $X$. The lemma describes a set of sufficient conditions which allow one to find such paths.

Lemma B.3 Let $k, l$ be non-negative integers. Let $G$ be a graph and $X \subseteq V(G)$ a subset of vertices with a distance function $d$ defined on $X$. Let $v$ be a vertex in $X$ such that $d(v, x) \geq l$ for all $x \in X \backslash\{v\}$. Let $P_{1}, \ldots, P_{k-1}$ be disjoint $(X \backslash\{v\})$-paths in $G-v$ where the endpoints of $P_{i}$ are $x_{i}$ and $y_{i}$ for $1 \leq i \leq k-1$. Assume that $d\left(x_{i}, y_{i}\right) \geq k l$ for all $1 \leq i \leq k-1$, and, furthermore, assume there exist $2 k-1 X$-paths with pairwise intersecting only in the vertex $v$. Then there exist disjoint $X$-paths $P_{1}^{\prime}, \ldots, P_{k}^{\prime}$ such that

i. the endpoints of $P_{i}^{\prime}$ are $x_{i}^{\prime}$ and $y_{i}^{\prime}$ and $d\left(x_{i}^{\prime}, y_{i}^{\prime}\right) \geq l$ for all $1 \leq i \leq k$, and

ii. there exists $x \in X$ such that $x_{i}^{\prime}, y_{i}^{\prime} \in\left\{x_{1}, \ldots, x_{k-1}, y_{1}, \ldots, y_{k-1}, v, x\right\}$ for all $1 \leq i \leq k$. 
Proof. We proceed by induction on $k$. When $k=0$, the claim is trivially true. Thus, we may assume $k \geq 1$. Let $Q_{1}, \ldots, Q_{2 k-1}$ be $X$-paths such that $Q_{i} \cap Q_{j}=v$ for all $i \neq j$. Pick such paths to minimize $\bigcup_{i=1}^{k-1} E\left(P_{i}\right) \cup \bigcup_{i=1}^{2 k-1} E\left(Q_{i}\right)$. We may assume that $G=\bigcup_{1}^{2 k-1} Q_{i} \cup \bigcup_{1}^{k-1} P_{i}$, as any additional edges or vertices may be deleted and we still satisfy the hypotheses.

First, we observe that for every index $i, 1 \leq i \leq k-1$, the path $P_{i}$ intersects $Q_{j}$ for at least 2 distinct indices $j$. Assume that there exists an index $\alpha$ such that at most 1 index $j$ satisfies $P_{\alpha} \cap Q_{j} \neq \emptyset$. By applying the induction hypothesis to the graph $\bigcup_{\left\{j \mid P_{\alpha} \cap Q_{j}=\emptyset\right\}} Q_{j} \cup \bigcup_{\{i \neq \alpha\}} P_{i}$, we find $k-1 X$-paths satisfying $i$ and $i$. By construction, the $k-1$ paths we find will be disjoint from $P_{\alpha}$. Thus, by including $P_{\alpha}$, we find the desired $k$ paths satisfying $i$ and $i i$.

We claim that for every index $i, 1 \leq i \leq k-1$, there exist indices $j$ and $j^{\prime}$ such that $x_{i}$ is an end of $Q_{j}$ and $y_{i}$ is an endpoint of $Q_{j^{\prime}}$. Fix an index $i$ such that $x_{i}$ is not contained in $Q_{j}$ for all indices $j$ in order to reach a contradiction. As we have already seen, $P_{i}$ intersects at least two distinct paths $Q_{j}$. Let $w$ be the first vertex of $\bigcup_{j=1}^{2 k-1} V\left(Q_{j}\right)$ which we encounter when traversing $P_{i}$ from $x_{i}$ to $y_{i}$. Let $\beta$ be such that $w \in V\left(Q_{\beta}\right)$. If we replace $Q_{\beta}$ with the path $v Q_{\beta} w P_{i} x_{i}$, we find $2 k-1$ paths from $v$ to $X \backslash\{v\}$ intersecting only in the vertex $v$. Moreover, if we let $x$ be the end of $Q_{\beta}$ in $X \backslash\{v\}$, we see that $w Q_{\beta} x$ cannot be equal to $w P_{i} y_{i}$, as $w P_{i} y_{i}$ must intersect a second path $Q_{j}$. Thus, the subpath $w Q_{\beta} x$ must contain at least one edge not contained in $\bigcup_{j=1}^{k-1} E\left(P_{j}\right)$. This contradicts our choice of $Q_{1}, \ldots, Q_{2 k-1}$ to minimize $\bigcup_{j=1}^{k-1} E\left(P_{j}\right) \cup \bigcup_{j=1}^{2 k-1} E\left(Q_{j}\right)$ and proves the claim.

Without loss of generality, we assume $Q_{2 k-1}$ has no endpoint in $\left\{x_{1}, \ldots, x_{k-1}, y_{1}, \ldots, y_{k-1}\right\}$. Let $r$ be the endpoint of $Q_{2 k-1}$ not equal to $v$. We conclude that $X=\left\{x_{1}, \ldots, x_{k-1}, y_{1}, \ldots, y_{k-1}, v, r\right\}$.

We define an auxiliary graph $H$ with vertex set $\left\{x_{1}, \ldots, x_{k-1}, y_{1}, \ldots, y_{k-1}, v, r\right\}$ such that two vertices are adjacent if and only if they have distance less than $l$ with the distance function $d$. Consider the components of this graph. First, note that the vertex $v$ has no neighbors and is an isolated vertex in $H$. Observe that for all indices $i, 1 \leq i \leq k-1$, it is not the case that both $x_{i}$ and $y_{i}$ are contained in the same component of $H$. Otherwise, we pick such an index $i$ and a path in $H$ connecting $x_{i}$ and $y_{i}$ chosen over all such indices to minimize the length of the path. The path cannot contain $v$, as $v$ is an isolated vertex in $H$. It follows that the path has at most $k$ edges. Given that any two adjacent vertices in $H$ have distance at most $l-1$, the triangle inequality implies that $x_{i}$ and $y_{i}$ have distance at most $k(l-1)<k l$, contradicting our assumptions.

Let $\mathcal{S}$ be the partition of $X$ given by the connected components of $H$. We apply Theorem B.2 to $G$ with the partition $\mathcal{S}$ of $X$. If there exist $k$ disjoint $\mathcal{S}$-paths in $G$, such paths would satisfy $i$ and $i i$, proving the theorem. Thus we assume, in order to reach a contradiction, that there exists a partition $U_{0}, U_{1}, \ldots, U_{n}$ of the vertex set of $G$ such that

$$
\left|U_{0}\right|+\sum_{1}^{n}\left\lfloor\frac{1}{2}\left|B_{i}\right|\right\rfloor \leq k-1
$$

Note that in fact we have equality above, as $P_{1}, \ldots, P_{k-1}$ form $k-1$ disjoint $\mathcal{S}$-paths. Also, note that each of the paths $Q_{i}$ for $1 \leq i \leq 2 k-1$ is an $\mathcal{S}$-path.

Recall that $B_{i}$ is the set of vertices of $U_{i} \cap X$ along with the vertices of $U_{i}$ with a neighbor in $V(G) \backslash\left(U_{0} \cup U_{i}\right)$. Observe that if $R$ is an $X$-path which is equal to $P_{i}$ for some $1 \leq i \leq k-1$ or $R=Q_{j}$ for some $1 \leq j \leq 2 k-1$, then for all $l, 1 \leq l \leq n$, we have the property that if both $V(R) \cap U_{0}=\emptyset$ and $\left|U_{i} \cap V(R)\right| \geq 2$, then we can conclude that $\left|B_{i} \cap V(R)\right| \geq 2$.

We now show that $v \notin U_{0}$. Fix $J \subseteq\{1, \ldots, k-1\}$ such that $V\left(P_{j}\right) \cap U_{0} \neq \emptyset$ if and only if $j \in J$. By the definition of $B_{i}$, for every $j \notin J$ there exists an index $i \geq 1$ such that $\left|U_{i} \cap V\left(P_{j}\right)\right| \geq 2$, and consequently, 
$\left|B_{i} \cap V\left(P_{j}\right)\right| \geq 2$. Combining these bounds, we see that

$$
\begin{aligned}
k-1 & =\left|U_{0}\right|+\sum_{i=1}^{n}\left\lfloor\frac{1}{2}\left|B_{i}\right|\right\rfloor \\
& \geq\left|U_{0} \cap\{v\}\right|+\left|U_{0} \cap\left(\bigcup_{i=1}^{k-1} V\left(P_{i}\right)\right)\right|+\sum_{i=1}^{n} \sum_{j \notin J}\left\lfloor\frac{1}{2}\left|B_{i} \cap V\left(P_{j}\right)\right|\right\rfloor \\
& \geq\left|U_{0} \cap\{v\}\right|+|J|+(k-1-|J|) \\
& \geq\left|U_{0} \cap\{v\}\right|+k-1 .
\end{aligned}
$$

Specifically, we conclude that equality holds throughout and $\left|U_{0} \cap\{v\}\right|=0$. Without loss of generality, we assume that $v \in U_{1}$.

We re-iterate the above argument with the paths $Q_{1}, \ldots, Q_{2 k-1}$. Let $J_{0} \subseteq\{1, \ldots, 2 k-1\}$ be such that $V\left(Q_{j}\right) \cap U_{0} \neq \emptyset$ if and only if $j \in J_{0}$. Let $J_{1} \subseteq\{1, \ldots, 2 k-1\} \backslash J_{0}$ be the set of indices $j$ such that such that $\left|V\left(Q_{j}\right) \cap U_{1}\right| \geq 2$. We see that

$$
\begin{aligned}
k-1 & =\left|U_{0}\right|+\sum_{i=1}^{n}\left\lfloor\frac{1}{2}\left|B_{i}\right|\right\rfloor \\
& \geq\left|J_{0}\right|+\left\lfloor\frac{1}{2}\left(\left|J_{1}\right|+1\right)\right\rfloor+\sum_{i=2}^{n} \sum_{j \notin\left(J_{0} \cup J_{1}\right)}\left\lfloor\frac{1}{2}\left|B_{i} \cap V\left(Q_{j}\right)\right|\right\rfloor \\
& \geq\left|J_{0}\right|+\left\lfloor\frac{1}{2}\left(\left|J_{1}\right|+1\right)\right\rfloor+(2 k-1)-\left|J_{0}\right|-\left|J_{1}\right| \\
& \geq(2 k-1)+\frac{1}{2}\left(\left|J_{1}\right|+1\right)-\frac{1}{2}-\left|J_{1}\right| \\
& \geq(2 k-1)-\frac{\left|J_{1}\right|}{2} \geq k-\frac{1}{2},
\end{aligned}
$$

a contradiction. Note, we use the fact that $v \notin U_{0}$ to claim that $\left|U_{0}\right| \geq\left|J_{0}\right|$. This contradiction shows that no such partition $U_{0}, \ldots, U_{1}$ of $V(G)$ exists, and completes the proof of the lemma.

\section{Rerouting patterns in a planar annulus}

We remind the reader that a linkage $\mathcal{P}^{\prime}$ is equivalent to a linkage $\mathcal{P}$ if they have the same number of components and for every component $P \in \mathcal{P}$, there exists a component $P^{\prime} \in \mathcal{P}^{\prime}$ such that $P$ and $P^{\prime}$ have the same endpoints.

Let $\mathcal{P}$ be a pattern in a society $(G, \Omega)$. Then $\mathcal{P}$ is crooked if for every $P \in \mathcal{P}$, both segments of $\Omega-V(P)$ contain some vertex of $V(\mathcal{P}) \cap \Omega$. Note that a $k$-crosscap is a crooked pattern trivially, and the fact that both a $(k, l)$-cross pattern and $(k, l)$-leap pattern are crooked patterns follows by the minimality assumption in their respective definitions.

We will need several definitions concerning sets of disjoint cycles in nearly flat decompositions.

Definition Let $(G, \Omega)$ be a society with a 1-nearly flat decomposition $\left(H_{0}, H_{1}, \ldots, H_{n}\right)$ for $n \geq 1$. Let $\Delta$ be the disc, $\Delta_{i}$ for $1 \leq i \leq n$ be the open discs as in the definition, and fix an embedding of $H_{0}$ in $\Delta-\left(\bigcup_{i=1}^{n} \Delta_{i}\right)$. Let $s \geq 1$ be a positive integer. The cycles $C_{1}, C_{2}, \ldots, C_{s}$ in $G$ form an $s$-nest if the following conditions are satisfied. 
i. For all $1 \leq i<j \leq s, V\left(C_{i}\right) \cap V\left(C_{j}\right)=\emptyset$.

ii. For each small vortex $\left(H_{i}, \Omega_{i}\right), i \geq 2$ and for all $1 \leq j \leq s$, we have that $\left|V\left(C_{j}\right) \cap \Omega_{i}\right| \leq 2$.

For every $1 \leq i \leq s$, let $\bar{C}_{i}$ be cycle of $\bar{H}_{0}$ given by $C_{i} \cap H_{0}$ as well as at most one edge of $\bar{H}_{0}\left[V\left(H_{j}\right) \cap V\left(H_{0}\right)\right]$ for $2 \leq j \leq n$. By ii. above, the cycle $\bar{C}_{i}$ is well defined for every $1 \leq i \leq s$.

iii. For each $1 \leq i \leq s$, the cycle $\bar{C}_{i}$ bounds a disc $\bar{\Delta}_{i} \subseteq \Delta$ such that $\Delta \supseteq \bar{\Delta}_{s} \supseteq \cdots \supseteq \bar{\Delta}_{1}$.

Definition Let $C_{1}, \ldots, C_{s}$ be pairwise disjoint cycles in a society $(G, \Omega)$, and let $\mathcal{P}$ be a pattern. We say that $\mathcal{P}$ is orthogonal to $C_{1}, \ldots, C_{s}$ if for every $P \in \mathcal{P}$ with endpoints $x$ and $y$ in $\Omega$, there exists a vertex $z \in V(P)$ such that the following holds:

i. for $1 \leq i \leq s$, both $x P z \cap C_{i}$ and $z P y \cap C_{i}$ form connected subpaths of $C_{i}$, and

ii. $x P z$ intersects each of the cycles $C_{s}, C_{s-1}, \ldots, C_{1}$ in that order and $z P y$ intersects each cycle $C_{1}, C_{2}, \ldots, C_{s}$ in that order.

The first result of this section shows when we can find many cycles which are orthogonal to a given pattern. If our society is 1-nearly flat, we will see that it suffices to contain a huge nest. In order to state this result, we will need the following theorem.

Theorem C.1 ([42], see also [17]) For all $k \geq 1$, there exists a value $w(k)$ such that the following holds. Let $\mathcal{P}$ be a linkage of order $k$ in a graph $G$ with $V(G)=V(\mathcal{P})$. Then either there exists a vertex $v$ in $G$ and a linkage $\mathcal{P}^{\prime}$ in $G-v$ which is equivalent to $\mathcal{P}$, or the treewidth of $G$ is at most $w(k)$.

The next theorem is a restatement of Theorem 10.1 in [16].

Theorem C.2 (10.1, [16]) Let $(G, \Omega)$ be a society and let $k, t \geq 1$ be positive integers. Let $\mathcal{P}$ be a crooked pattern of order $k$. Let $s=w(k)+t$ where $w(k)$ is the function in Theorem C.1. Let $H_{0}, H_{1}, \ldots, H_{n}$ be a 1-nearly flat decomposition of $(G, \Omega)$ such that all the small vortices are grounded. If there exists an s-nest $C_{1}, \ldots, C_{s}$, then there exist a $t$-nest $C_{1}^{\prime}, \ldots, C_{t}^{\prime}$ in $G$ and a pattern $\mathcal{P}^{\prime}$ equivalent to $\mathcal{P}$ such that $\mathcal{P}^{\prime}$ and $C_{1}^{\prime}, \ldots, C_{t}^{\prime}$ are orthogonal.

In our proofs, we will inductively find a large crooked transaction in a smaller society. We will then need to find a pattern which is substantially the same, but avoids a fixed segment of the society vertices. First, we define what we mean by "substantially" the same for two crooked patterns. Let $\mathcal{P}$ and $\mathcal{P}^{\prime}$ be two patterns in a society $(G, \Omega)$ such that the components of $\mathcal{P}$ are $P_{1}, \ldots, P_{k}$ and the endpoints of $P_{i}$ equal to $s_{i}$ and $t_{i}$. The patterns $\mathcal{P}$ and $\mathcal{P}^{\prime}$ are homeomorphic if there exists a labeling $P_{1}^{\prime}, \ldots, P_{k}^{\prime}$ of the components of $\mathcal{P}^{\prime}$ and their endpoints such that the endpoints of $P_{i}^{\prime}$ are equal to $s_{i}^{\prime}$ and $t_{i}^{\prime}$ and the vertices $s_{1}, \ldots, s_{k}, t_{1}, \ldots, t_{k}$ and $s_{1}^{\prime}, \ldots, s_{k}^{\prime}, t_{1}^{\prime}, \ldots, t_{k}^{\prime}$ both occur in $\Omega$ is the same cyclic order.

Now we consider the property for a given segment of society vertices that will allow us to find a homeomorphic pattern with no endpoint in that segment.

Definition Let $(G, \Omega)$ be a society, and let $S$ be a segment of the society. For all $t \geq 1$, we say that $S$ is $t$-insulated if there exists a separation $(A, B)$ of $G$ with the following properties:

i. $S \subseteq A$, and $A \cap \Omega$ and $B \cap \Omega$ form two segments of $\Omega$ intersecting in exactly two vertices, call them $v_{1}$ and $v_{n}$ for some $n \geq 2$.

ii. There exists an enumeration $v_{1}, v_{2}, \ldots, v_{n}$ of the vertices $A \cap B$ such that if we let $\Omega_{A}$ be the cyclic order of $(A \cap B) \cup(A \cap \Omega)$ given by $v_{1} \Omega v_{n} v_{n-1}, \ldots, v_{1}$, then $\left(G[A], \Omega_{A}\right)$ has a 0 -nearly flat decomposition. 
iii. If we let $X$ and $Y$ be the two segments of $(\Omega \cap A) \backslash S$, there exist $t$ disjoint paths in $G[A]$ from $X$ to $Y$.

Now we state the theorem. The proof is similar to the proof of Theorem C.2.

Theorem C.3 Let $(G, \Omega)$ be a society and let $\mathcal{P}$ be a crooked pattern in $(G, \Omega)$ of order $k$. Let $S$ be a segment of $\Omega$. Let $w$ be the function in Theorem C.1. If $S$ is $(2 k+1)(2 k+2 w(k))$-insulated, then there exists a pattern $\overline{\mathcal{P}}$ which is homeomorphic to $\mathcal{P}$ which does not have any endpoint in $S$.

Proof. Fix $t=2 k+2 w(k)$, and let $(A, B)$ be the separation as in the definition of $(2 k+1) t$-insulated with $S \subseteq A$. Let $X$ and $Y$ be the two segments of $\Omega$ comprising the vertices $(A \cap \Omega) \backslash S$. Then in $G[A]$, there exist $(2 k+1) t$ disjoint paths from $X$ to $Y$. Since there are at most $2 k$ vertices of $\Omega \cap V(\mathcal{P})$ which are contained in $X \cup Y$, it follows that we can find segments $\bar{X} \subseteq X$ and $\bar{Y} \subseteq Y$ which are disjoint from $V(\mathcal{P})$ and a pattern $\mathcal{Q}$ of order $t$ contained in $G[A]$ such that every component has one endpoint in $\bar{X}$ and one endpoint in $\bar{Y}$.

Fix $\mathcal{P}^{\prime}$ to be a pattern contained in $G$ equivalent to $\mathcal{P}$ and $\mathcal{Q}^{\prime}$ a pattern in $G[A]$ equivalent to $\mathcal{Q}$ which minimize $E\left(\mathcal{P}^{\prime}\right) \cup E\left(\mathcal{Q}^{\prime}\right)$. Let $G^{\prime}$ be defined to be the subgraph $\mathcal{P}^{\prime} \cup \mathcal{Q}^{\prime}$ and let $\Omega^{\prime}$ be the cyclic order $\Omega$ restricted to $\Omega \cap\left(V\left(\mathcal{P}^{\prime}\right) \cup V\left(\mathcal{Q}^{\prime}\right)\right)$. We will in fact see that the desired pattern $\overline{\mathcal{P}}$ is contained in $G^{\prime}$.

Let $\Omega_{A}$ be the cyclic order on a subset of $A$ as in the definition of $t$-insulated. Let $A^{\prime}=A \cap V\left(G^{\prime}\right)$ and $B^{\prime}=B \cap V\left(G^{\prime}\right)$, and $\Omega_{A^{\prime}}$ be the cyclic order $\Omega_{A}$ restricted to $A^{\prime}$. Given that $\left(G[A], \Omega_{A}\right)$ has a 0 -flat decomposition, it follows that $\left(G^{\prime}\left[A^{\prime}\right], \Omega_{A^{\prime}}\right)$ does as well. Fix $H_{0}, H_{1}, \ldots, H_{n}$ to be a 0 -flat decomposition of $\left(G^{\prime}\left[A^{\prime}\right], \Omega_{A^{\prime}}\right)$. By Observation 2, we may assume that the vortices of the decomposition are grounded. We pick our grounded decomposition to minimize $n$, the number of vortices.

We begin with several claims about the the 0-near flat decomposition.

Claim C.4 There are no vortices in the decomposition and $G^{\prime}\left[A^{\prime}\right]=H_{0}$.

Proof. Assume $H_{1}$ is defined. Since the decomposition is 0-nearly flat, it follows that $\left|V\left(H_{0}\right) \cap V\left(H_{1}\right)\right| \leq 3$. Let $x_{1}, \ldots, x_{l}$ be the vertices of $V\left(H_{0}\right) \cap V\left(H_{1}\right)$ for some integer $l, 1 \leq l \leq 3$. Let $\Delta_{1}$ be the open disc of the disc $\Delta$ corresponding to $H_{1}$ in the definition of a 0 -near decomposition.

First, observe that there exists at most one component of each of $\mathcal{P}^{\prime}$ and $\mathcal{Q}^{\prime}$ that intersect $E\left(H_{1}\right)$ by the fact that $E\left(H_{1}\right)$ is separated from the rest of $G^{\prime}$ by a 3-cut. Moreover, given that $V\left(G^{\prime}\right)=\mathcal{P}^{\prime} \cup \mathcal{Q}^{\prime}$, we see that at least one such path does intersect $V\left(H_{1}\right) \backslash V\left(H_{0}\right)$. If $l \leq 2$, it follows that $l=2$ and $H_{1}$ consists of just a single path linking the vertices $x_{1}$ and $x_{2}$ by our choice to minimize the edges of $G^{\prime}$. Let $\Delta$ be the disc and let $\Delta_{1}, \ldots, \Delta_{n}$ be the discs as in the definition of 0-nearly flat decomposition. We see that $H_{1} \cup H_{0}$ can be embedded in $\Delta-\bigcup_{i=2}^{n} \Delta_{i}$ by embedding $H_{1}$ in $\Delta_{1}$. This contradicts our choice to minimize the value $n$. Similarly, if $l=3$, given minimality and the fact that $H_{1}$ is grounded, we can show the following after possibly re-labeling the vertices of $V\left(H_{1}\right) \cap V\left(H_{0}\right)$. The subgraph $H_{1}$ must consist of exactly the union of a subpath $P$ of $\mathcal{P}$ linking $x_{1}$ and $x_{2}$ and a subpath $Q$ with one end equal to $x_{3}$ and the other endpoint contained in $V(P)$. It follows that $H_{0} \cup H_{1}$ can again be embedded in $\Delta-\bigcup_{i=2}^{n} \Delta_{i}$, contrary to our choice to minimize $n$.

Claim C.5 There does not exist a component $P$ of $\mathcal{P}^{\prime}$ with $V(P) \subseteq A^{\prime}$.

Proof. Pick such a component $P$ and a segment $Z$ of $\Omega_{A^{\prime}}$, to minimize $|Z|$, with $Z \subseteq \Omega \cap A$ such that $Z$ contains the endpoints of $P$. Given that $A^{\prime}$ can be embedded on the disc, we see that $V(P)$ is a cutset separating $Z$ from $\Omega_{A^{\prime}} \backslash Z$. However, by the fact that $\mathcal{P}^{\prime}$ is a crooked pattern, there exists some component $P_{2}$ of $\mathcal{P}$ which intersects $Z \backslash V(P)$. It follows that $P_{2}$ is contained in $G^{\prime}\left[A^{\prime}\right]$ and has both endpoints in $Z \backslash V(P)$. This, however, contradicts our choice of $P$ and $Z$ to minimize $|Z|$, proving the claim.

Finally, we see $\mathcal{P}^{\prime}$ and $\mathcal{Q}^{\prime}$ form a subgraph of bounded treewidth. 
Claim C.6 The graph $\mathcal{P}^{\prime} \cup \mathcal{Q}^{\prime}$ has treewidth $t w\left(G^{\prime}\right)<w(k)$.

Proof. If there exists a linkage $\mathcal{P}^{\prime \prime}$ equivalent to $\mathcal{P}$ which avoids some vertex of degree at least 3 in $G^{\prime}$, it follows that $\mathcal{P}^{\prime \prime} \cup \mathcal{Q}^{\prime}$ will have strictly fewer edges, contradicting our choice of $\mathcal{P}^{\prime}$ and $\mathcal{Q}^{\prime}$. Note that every vertex of $\Omega \cap V\left(\mathcal{Q}^{\prime}\right)$ has degree one in $G^{\prime}$, and so each component of $\mathcal{P}^{\prime \prime}$ intersects $\Omega$ exactly in its endpoints. Consequently, $\mathcal{P}^{\prime \prime}$ is a pattern as required. The claim follows now by suppressing vertices of degree at most 2 in $G^{\prime}$ and applying Theorem C.1.

We number the components of $\mathcal{Q}^{\prime} Q_{1}, Q_{2}, \ldots, Q_{t}$ so that $Q_{1}$ is "closest" to the segment $S$, followed by $Q_{2}$, and so on. Explicitly, there exist segments $S_{0}=S \subseteq S_{1} \subseteq S_{2} \subseteq \cdots \subseteq S_{t}$ of $\Omega$ such that $Q_{i}$ has both endpoints in $S_{i}$ and $V\left(Q_{i}\right) \cap S_{i+1}=\emptyset$. Then, by our choice of $\mathcal{P}^{\prime}$ and $\mathcal{Q}^{\prime}$ to minimize the number of their edges, we see that there does not exist a subpath $R$ of $\mathcal{P}^{\prime} \cap G^{\prime}\left[A^{\prime}\right]$ with both endpoints in some component $Q_{i}$ of $\mathcal{Q}^{\prime}$ and which is otherwise disjoint from $\mathcal{Q}^{\prime}$. Otherwise, we could reroute $Q_{i}$ through $R$ and reduce the number of edges in their union.

We define a local peak to be a subpath $R$ of $\mathcal{P}^{\prime}$ such that $R$ has both endpoints in $Q_{i}$ for some index $i<t$, at least one internal vertex in $Q_{i+1}$, and no internal vertex in $\mathcal{Q}^{\prime}-Q_{i+1}$.

Claim C.7 There does not exist a local peak.

Proof. Assume such a local peak $R$ exists with its endpoints are contained in $Q_{i}$, and choose such a local peak to minimize the value $i$. Assume $R$ is contained in the component $P$ of $\mathcal{P}^{\prime}$. Let the endpoints of $R$ be $x$ and $y$. Then the subpath $x Q_{i} y$ must intersect some component of $\mathcal{P}^{\prime}$, otherwise we could reroute $P$ through $Q_{i}$ and reduce the number of edges in the union. Let $P_{2}$ be a component of $\mathcal{P}^{\prime}$ intersecting $x Q_{i} y$. Given that $P_{2}$ does not have endpoints in $(\bar{X} \cup \bar{Y}) \cap \Omega_{A^{\prime}}$, if $i>1$, it follows that $P_{2}$ has a subpath forming a local peak with endpoints on $Q_{i-1}$, contrary to our choice of local peak. However, if $i=1$, it follows that either $P_{2}$ has a subpath with both endpoints contained in $Q_{1}$ and no internal vertex in $\mathcal{Q}^{\prime}$, or, $P_{2}$ is strictly contained in $G^{\prime}\left[A^{\prime}\right]$. In the first case, we can reroute $Q_{1}$ to reduce the number of edges in the union; the second case contradicts Claim C.5. This proves the claim.

We define a dive to be a subpath of $\mathcal{P}\left[A^{\prime}\right]$ with both endpoints in $\Omega_{A^{\prime}} \cap\left(A^{\prime} \cap B^{\prime}\right)$. Let $R$ be a dive with endpoints $x$ and $y$. Observe that by Claim C.7, there exist an index $i$ and a vertex $z$ in $V(R) \cap V\left(Q_{i}\right)$ such that the subpath $x R z$ (and symmetrically, $z R y$ ) intersects the path $Q_{j}$ in a subpath of $Q_{j}$ for all $i \leq j \leq s$. Moreover, the subpath $x R z$ (and again symmetrically, $z R y$ ) intersects each of the paths $Q_{t}, Q_{t-1}, \ldots, Q_{i}$ in order when traversing $x R z$ from $x$ to $z$. Thus, the path $R$ "dives" directly down to a "deepest" path $Q_{i}$, and then returns as directly as possible to $A^{\prime} \cap B^{\prime}$.

We define the depth of a dive $R$ to be the minimal index $i$ such that $V(R) \cap V\left(Q_{i}\right) \neq \emptyset$.

Claim C.8 There does not exist a dive of depth $2 w(k)$.

Proof. We first observe that if there exists a dive $R$ of depth $l$, then there must exist a dive $R^{\prime}$ of depth $l-1$, otherwise we could reroute the component of $\mathcal{P}^{\prime}$ containing $R$ through a subpath of $Q_{i+1}$ and reduce the number of edges. It follows that if there exists a dive of depth $2 w(k)$, then there exist dives of depth $i$ for all $w(k) \leq i \leq 2 w(k)$. It follows that the union of the dives and the paths $Q_{t}, Q_{t-1}, \ldots, Q_{t-w(k)}$ contains a $w(k) \times w(k)$-grid minor, contradicting Claim C.6 because a $w(k) \times w(k)$-grid minor has treewidth at least $w(k)$ (see [10]). This proves the claim.

We conclude that at most $2 k$ components of $\mathcal{P}^{\prime}\left[A^{\prime}\right]$ intersect the paths $Q_{1}, \ldots, Q_{2 k}$. Moreover, by Claim C.7, they must intersect in a clean manner. It is now an easy matter to find a pattern homeomorphic to $\mathcal{P}^{\prime}$ by rerouting the components of $\mathcal{P}^{\prime}$ which intersect $Q_{1}, \ldots, Q_{2 k}$ so that they have their endpoints contained in $\bar{X}$. This completes the proof of the theorem. 


\section{Proof of Theorem A.6}

In this section we present the proof of the main society theorem, Theorem A.6. We first need several quick results going forward. The first is a theorem of Erdős and Szekeres.

Theorem D.1 Let $k \geq 1$ be a positive integer, and let $a(1), a(2), \ldots, a\left(k^{2}\right)$ be a sequence of distinct integers of length $k^{2}$. Then there exists either a monotone increasing or monotone decreasing subsequence of length $k$.

We will also need the following lemma. The statement is somewhat technical, but describes a situation which will arise in the proof.

Lemma D.2 Let $G$ be a graph and $X$ and $Y$ two nonempty subsets of vertices. Let $k \geq 1$ be a positive integer. Assume that there exists at least one $X-Y$ path in $G$, but there do not exist $k$ vertex disjoint $X-Y$ paths. Then there exists a separation $(A, B)$ with $X \subseteq A$ and $Y \subseteq B$ satisfying exactly one of the following:

a. $|A \cap B|=k$, for all $z \in A \cap B$ there exists a $z-X$ path in $G[A]$, and there exist $k$ disjoint $(A \cap B)-Y$ paths in $G[B]$, or

b. $|A \cap B|<k$ and $(A \cap B) \subseteq Y$.

Proof. The proof proceeds by induction on $|V(G) \backslash X|$. Note that if $X=V(G)$, then it follows that $|Y| \leq k$, lest there exist $k$ trivial $X-Y$ paths, and consequently, the trivial separation $(V(G), Y)$ satisfies b.

We assume that there do not exist $k$ disjoint $X-Y$ paths. Let $(A, B)$ be a separation of minimal order with $X \subseteq A$ and $Y \subseteq B$, chosen over all such minimal separations to minimize $|B|$. Note that by assumption, $(A, B)$ has order at least one. Also, by minimality, for every vertex $z$ in $A \cap B$ there exists an $X-z$ path in $G[A]$. Assume that $B \backslash A \neq V(G) \backslash X$. If we consider the subgraph $G[B]$, there do not exist $k$ disjoint $(A \cap B)-Y$ paths, and there exists at least one such path. By the induction hypothesis applied to $G[B]$ and the two sets $A \cap B$ and $Y$, there exists a separation $\left(A^{\prime}, B^{\prime}\right)$ of $G[B]$ with $A \cap B \subseteq A^{\prime}$ and $Y \subseteq B^{\prime}$ satisfying either $a$. or $b$. It follows that $\left(A \cup A^{\prime}, B^{\prime}\right)$ is a separation in $G$ satisfying $a$. or $b$., as desired.

Thus we have reduced to the case when $B \backslash A=V(G) \backslash X$. Then by our choice of separation $(A, B)$, there does not exist any other order $|A \cap B|$ separation separating $X$ and $Y$. We may assume that $A \cap B \nsubseteq Y$, lest the separation $(A, B)$ satisfy $b$. Let $x$ be a vertex of $B \backslash A$ which is adjacent to a vertex of $A \cap B$. Such a vertex exists because there exist $|A \cap B|$ disjoint paths from $A \cap B$ to $Y$ by our choice of a minimal order separation. Given that $A \cap B \nsubseteq Y$, we may simply pick the next vertex on one of these paths. We see that there exist $|A \cap B|+1$ disjoint paths from $(A \cap B) \cup\{x\}$ to $Y$ by the fact that $(A, B)$ was the unique order $|A \cap B|$ separation separating $X$ and $Y$. If $|A \cap B|+1=k$, then the separation $(A \cup\{x\}, B)$ satisfies $a$. If instead $|A \cap B|+1<k$, then we apply induction to $G[B]$ with the two sets $(A \cap B) \cup\{x\}$ and $Y$ to find a separation $\left(A^{\prime}, B^{\prime}\right)$ satisfying $a$. or $b$. The separation $\left(A \cup A^{\prime}, B\right)$ then satisfies one of the desired outcomes in the original graph.

In the proof we will need to briefly consider the bridges of a linkage. We recall that a bridge of a linkage $\mathcal{P}$ in a graph $G$ is a subgraph $B$ such that $B$ is either an edge with both endpoints in $V(\mathcal{P})$ (known as a trivial bridge), or $B-V(\mathcal{P})$ is a connected component of $G-V(\mathcal{P})$ and $B$ contains every edge with one endpoint in $V(\mathcal{P})$ and one endpoint in $V(B) \backslash V(\mathcal{P})$. Thus the bridges can be thought of the distinct "pieces" of the graph attaching to the linkage $\mathcal{P}$.

The proof of Theorem A.6 will be an inductive proof on the value $k$. The inductive step hinges on finding a large, almost planar subgraph in the society. We state this as a separate lemma.

Lemma D.3 Let $k, l \geq 2$, and $t$ be non-negative integers such that $t \geq l, t \geq k$. Let $(G, \Omega)$ be a society. There exists a value $\alpha=\alpha(k, t)$ such that one of the following holds. 
1. $(G, \Omega)$ contains a $k$-crosscap, a $(k, l)$-cross pattern, or a $(k, l)$-leap pattern, or

2. $(G, \Omega)$ admits a vortex decomposition of adhesion $\alpha$, or

3. there exists a set $Z \subseteq V(G)$ with $|Z| \leq 4 k^{2}$, segments $S_{1}, S_{2}$ of $\Omega$ which are disjoint from $Z$, and a decomposition of $G-Z$ into graphs $G_{L}, H, G_{R}$ such that the following hold:

a. $V(H) \cap \Omega=S_{1} \cup S_{2}$. We can label the two segments of $\Omega \backslash\left(S_{1} \cup S_{2}\right)$ as $S_{L}$ and $S_{R}$ such that $V\left(G_{L}\right) \cap \Omega=S_{L} \backslash Z$ and $V\left(G_{R}\right) \cap \Omega=S_{R} \backslash Z$.

Let $\Omega_{H}$ be the cyclic order on $S_{1} \cup S_{2}$ induced by $\Omega$.

b. $\left(H, \Omega_{H}\right)$ has a 0-nearly flat decomposition $H_{0}, \ldots, H_{n}$ for some integer $n$.

c. $H$ contains $t$ disjoint $S_{1}-S_{2}$ paths $L_{1}, \ldots, L_{t}$. If we label the endpoints of $L_{i}$ in $S_{1}$ as $x_{i}$ for $1 \leq i \leq t$, then $x_{1}, x_{2}, \ldots, x_{t}$ occur on $S_{1}$ in that order.

d. For every vertex $v$ in $H$, there exists a path in $H$ connecting $v$ to $S_{1} \cup S_{2}$.

e. $V\left(G_{L}\right) \cap V(H) \subseteq V\left(L_{1}\right) \cap V\left(H_{0}\right)$ and $V\left(G_{R}\right) \cap V(H) \subseteq V\left(L_{t}\right) \cap V\left(H_{0}\right)$.

f. There is no $V\left(G_{L}\right)-V\left(G_{R}\right)$ path in $G-(Z \cup V(H))$.

Proof. We prove the lemma with

$$
\alpha=\left(t 4 k^{2}\right)^{2} .
$$

Assume $(G, \Omega)$ form a counterexample to the statement for the value of $\alpha$ above. If $(G, \Omega)$ admits a vortex decomposition of adhesion $\alpha$, then we immediately contradict our choice of $(G, \Omega)$ to be a counterexample. Thus, by Theorem A.3, we see that there exist disjoint segments $X$ and $Y$ in $\Omega$ and a pattern $\mathcal{L}$ of order $\alpha$ where each component in $\mathcal{L}$ has exactly one endpoint in $X$ and one endpoint in $Y$. There is a natural sequence associated to the pattern $\mathcal{L}$ obtained by ordering the components of $\mathcal{L}$ by the $\Omega$-order of their endpoints in $X$ and looking at their corresponding end in $Y$. By applying Theorem D.1 to this sequence, we see that there exists a sub-linkage $\mathcal{L}^{\prime}$ of the components of $\mathcal{L}$ of order $t 4 k^{2}$ such that the components of $\mathcal{L}^{\prime}$ are either pairwise crossing or pairwise non-crossing. If the components of $\mathcal{L}^{\prime}$ are pairwise crossing, then $\mathcal{L}^{\prime}$ contains a $k$-crosscap and the statement is proven.

By dividing $\mathcal{L}^{\prime}$ into sets of $t$ consecutive paths, we conclude that there exist $4 k^{2}$ distinct patterns $\mathcal{L}_{1}, \ldots, \mathcal{L}_{4 k^{2}}$ and segments $X_{1}, \ldots, X_{4 k^{2}} \subseteq X$ and $Y_{1}, \ldots, Y_{4 k^{2}} \subseteq Y$ satisfying the following.

- For all $1 \leq i<j \leq 4 k^{2}$, both $X_{i} \cap X_{j}=\emptyset$ and $Y_{i} \cap Y_{j}=\emptyset$.

- For all $1 \leq i \leq 4 k^{2}$, every component of $\mathcal{L}_{i}$ has one endpoint in $X_{i}$ and one end in $Y_{i}$.

- For all $1 \leq i \leq 4 k^{2}$, the components of $\mathcal{L}_{i}$ are pairwise non-crossing.

- The segments $X_{1}, \ldots, X_{4 k^{2}}$ occur in that order in $X$ if we look at the $\Omega$-order of the vertices of $X$. Similarly, the segments $Y_{4 k^{2}}, \ldots, Y_{1}$ occur in that order if we look at the $\Omega$-order of the vertices of $Y$.

Moreover, we assume that we have chosen inclusion-wise minimal segments $X_{1}, \ldots, X_{4 k^{2}}, Y_{1}, \ldots, Y_{4 k^{2}}$ with these properties. We let $A_{i}$ and $B_{i}$ be the two segments of $\Omega \backslash\left(X_{i} \cup Y_{i}\right)$. We label them such that $A_{1} \subseteq A_{2} \subseteq \cdots \subseteq A_{4 k^{2}}$

Given that $t \geq l$, we see that for all $1 \leq i \leq 4 k^{2}$ there exists a separation of order $k-1$ separating $A_{i}$ from $B_{i}$ in the graph $G-\left(X_{i} \cup Y_{i} \cup V\left(\mathcal{L}_{i}\right)\right)$, otherwise there would exist a $(k, l)$-leap pattern in $(G, \Omega)$. Let $Z_{i}$ be a cut set of size at most $k-1$ associated with such a separation.

For distinct $i$ and $j$, it is possible that $Z_{i} \cap V\left(\mathcal{L}_{j}\right) \neq \emptyset$. We pick a large collection of the linkages $\mathcal{L}_{i}$ such that their corresponding $Z_{i}$ 's are disjoint from the linkages. Explicitly, there exists a subset $I \subseteq\{1, \ldots, m\}$ with $|I| \geq m / k$ such that for all $i, j \in I, i \neq j$, we have that $Z_{i} \cap\left(X_{j} \cup Y_{j} \cup V\left(\mathcal{L}_{j}\right)\right)=\emptyset$. This follows since 
each $Z_{i}$ intersects at most $k-1$ sets of the form $X_{j} \cup Y_{j} \cup V\left(\mathcal{L}_{j}\right)$, implying that such a subset $I$ can simply be chosen greedily.

After possibly re-labeling the indices, we may assume $I=\{1,2, \ldots, 4 k\}$. We assume that the order of the patterns $\mathcal{L}_{i}$ is preserved, i.e. $A_{1} \subseteq A_{2} \subseteq \cdots \subseteq A_{4 k}$. We fix $Z=\bigcup_{i \in I} Z_{i}$.

For all $i, 1 \leq i \leq 4 k$, we define $\mathcal{B}_{i}$ to be the subgraph formed by the union of all $\bigcup_{1}^{4 k} \mathcal{L}_{j} \cup \Omega$ bridges in the graph $G-Z$ with at least one vertex contained in $X_{i} \cup Y_{i} \cup \mathcal{L}_{i}$. We now define the subgraph $H_{i}$ as

$$
\begin{aligned}
H_{i} & =X_{i} \cup Y_{i} \cup \mathcal{L}_{i} \cup \mathcal{B}_{i} \cup \\
& \cup\left\{L \mid L \text { a component of } \bigcup_{1}^{4 k} \mathcal{L}_{i} \text { and } V(L) \cap V\left(\mathcal{B}_{i}\right) \neq \emptyset\right\} .
\end{aligned}
$$

We define $\Omega_{i}$ to be the cyclic order given by $\Omega$ restricted to the vertices $V\left(H_{i}\right) \cap \Omega$.

The $H_{i}$ are not pairwise disjoint as constructed, but their intersections are limited. Specifically, we will see that if $i$ and $j$ are sufficiently far apart, then $H_{i} \cap H_{j}=\emptyset$. Towards that end, we observe that $\Omega_{i} \subseteq B_{i-2} \cap A_{i+2}$. Otherwise, by symmetry, we may assume that $\mathcal{B}_{i}$ contains an $\left(\Omega \cup \bigcup_{j=1}^{4 k} \mathcal{L}_{j}\right)$-bridge with one endpoint in $\mathcal{L}_{i}$ and one endpoint in $\bigcup_{j \geq i+2} \mathcal{L}_{j} \cup X_{i+2} \cup Y_{i+2} \cup B_{i+2}$. But then there exists a path from $A_{i+1}$ to $B_{i+1}$ in $G-Z$ which avoids the set of vertices $\mathcal{L}_{i+1} \cup X_{i+1} \cup Y_{i+1}$, contrary to our construction of the set $Z$.

We now apply Theorem A.4 to the society $\left(H_{i}, \Omega_{i}\right)$ for $1 \leq i \leq 4 k$. If there exist a pair of crossing paths $P$ and $Q$ in $\left(H_{i}, \Omega_{i}\right)$, then neither $P$ nor $Q$ crosses some component of the linkage $\mathcal{L}_{i+2}$, nor does either $P$ or $Q$ cross some component of the linkage $\mathcal{L}_{i-2}$. Thus, if $\left(H_{4 i}, \Omega_{4 i}\right)$ contains a pair of crossing paths $P_{4 i}, Q_{4 i}$ for all $1 \leq i \leq k$, we see that $P_{4}, \ldots, P_{4 k}, Q_{4}, \ldots, Q_{4 k}, \mathcal{L}_{6}, \mathcal{L}_{10}, \ldots, \mathcal{L}_{4 k-2}$ contains a $(k, l)$-cross pattern given that $t \geq \bar{l}$. We conclude that there exists an index $i$ such that $\left(H_{i}, \Omega_{i}\right)$ has a 0 -flat decomposition. Without loss of generality, we assume that $i=1$ and $\left(H_{1}, \Omega_{1}\right)$ has a 0 -flat decomposition. We label the components $L_{1}, L_{2}, \ldots, L_{t}$ such that the endpoints of $L_{i}$ are $x_{i}$ and $y_{i}$ for $1 \leq i \leq t$ and $x_{1}, x_{2}, \ldots, x_{t}$ occur on $X_{1}$ in that order and $y_{t}, \ldots, y_{1}$ occur on $Y_{1}$ in that order. We chose $X_{1}$ and $Y_{1}$ to be minimal by inclusion, and so we also see that $x_{1}$ is the first vertex on $X_{1}$, and $x_{t}$ the last. Similarly, $y_{t}$ is the first vertex on $Y_{1}$ and $y_{1}$ the last vertex. Let $H$ be the subgraph of $H_{1}$ given by $X_{1} \cup Y_{1} \cup V\left(\mathcal{L}_{1}\right)$ as well as the union of $\Omega \cup \bigcup_{1}^{4 k} \mathcal{L}_{i}$ bridges with at least one attachment contained in $\left(X_{1} \cup Y_{1} \cup \mathcal{L}_{1}\right)-\left(L_{1} \cup L_{t}\right)$, that is we eliminate bridges attaching only to the "external" paths $L_{1}$ and $L_{t}$. The 0 -flat decomposition of $\left(H_{1}, \Omega_{1}\right)$ induces a 0-flat decomposition on $\left(H, X_{1} \cup Y_{1}\right)$ as well by simply restricting the subgraphs of $H_{1}$ to subgraphs of $H$. Let $J_{0}, J_{1}, \ldots, J_{n}$ be the resulting decomposition for some non-negative integer $n$.

We let $S_{L}$ be the segment of $\Omega \backslash\left(X_{1} \cup Y_{1}\right)$ containing $A_{1}$, and we let $S_{R}$ be the segment of $\Omega \backslash\left(X_{1} \cup Y_{1}\right)$ containing $B_{1}$. Since there does not exist an $A_{1}-B_{1}$ path in $G-(Z \cup V(H))$, we let $\left(W_{L}\right.$, $\left.W_{R}\right)$ be a separation of order 0 with $A_{1} \subseteq W_{L}$ and $B_{1} \subseteq W_{R}$. We now define $G_{L}$ to be union of $G\left[W_{L}\right]$ as well as any edges with one end in $W_{L}$ and one end in $H$ as well as edges of $G-H$ with both ends in $L_{1}$. Similarly, we define $G_{R}$ to be the union of $G\left[W_{R}\right]$ and any edges with one end in $W_{R}$ and one end in $L_{t}$ as well as edges of $G-H$ with both ends in $L_{t}$. Note that $G_{R}, G_{L}$, and $H$ are pairwise edge disjoint by construction and $G_{R} \cup G_{L} \cup H=G-Z$.

We claim that the decomposition $G_{R}, H, G_{L}$ with $S_{1}=X_{1}$ and $S_{2}=Y_{1}$ satisfy outcome 3 in the statement, contrary to our choice of a counterexample. By construction, we clearly satisfy a. - d. as well as f. If we consider property e., the only vertices in common between $G_{L}$ and $H$ must belong to the path $L_{1}$. If these vertices are not contained in $J_{0}$, it follows that there exists an index $i$ such that $J_{i}$ consists exactly of a subpath of $L_{1}$. We can then appropriately embed $J_{0} \cup J_{i}$ in the disc so that the vertices of $G_{L} \cap H$ are embedded in the disc. Repeating this argument for all such vertices of $G_{L} \cap H$ and $G_{R} \cap H$ gives a 0 -flat decomposition satisfying e. as well, contradicting our choice of $(G, \Omega)$ to be a counterexample and completing the proof of the lemma.

We now proceed with the proof of Theorem A.6. In order to facilitate the induction, we will actually prove a slightly stronger statement. Theorem A.6 follows immediately by setting $k_{1}=k, k_{2}=k$, and $k_{3}=k$. 
Theorem D.4 For all non-negative integers $k_{1} \geq 2, k_{2} \geq 2, k_{3} \geq 0$ and $l \geq 2$, there exists a value $\alpha=$ $\alpha\left(k_{1}, k_{2}, k_{3}, l\right)$ satisfying the following. Let $(G, \Omega)$ be a society. Either

1. $(G, \Omega)$ contains a $k_{1}$-crosscap, a $\left(k_{2}, l\right)$-cross pattern, or a $\left(k_{3}, l\right)$-leap pattern, or

2. there exists a set $X \subseteq V(G)$ with $|X| \leq \alpha$ such that $(G-X, \Omega \backslash X)$ admits an $\alpha$-bounded $\left(k_{2}-1\right)$-nearly flat decomposition.

We quickly outline the proof. We proceed by induction on $\mid V(G)$ - and apply Lemma D.3 for some appropriately chosen big value $t$. After deleting the relatively small set $Z$ of vertices, we see that that the remaining graph can be divided into three pieces: the large nearly flat strip $H$ and two remaining societies, $G_{L}$ and $G_{R}$ glued onto $S_{L} \cup L_{1}$ and onto $S_{R} \cup L_{t}$ where the order of the vertices of $L_{1}$ and $L_{t}$ comes from the linear order given by the path. We would like to apply induction to each of the societies $\left(G_{L}, V\left(G_{L}\right) \cap\left(S_{L} \cup L_{1}\right)\right)$ and $\left(G_{R}, V\left(G_{R}\right) \cap\left(S_{R} \cup L_{t}\right)\right)$. If they both have the desired nearly flat decomposition, we can then glue the decompositions together in order to find a decomposition of the whole society. If they do not have the desired decompositions, we find $\left(k^{\prime}, l\right)$-cross pattern and a $\left(k^{\prime \prime}, l\right)$-cross pattern with $k^{\prime}+k^{\prime \prime} \geq k_{2}$ which can be combined to find a $\left(k_{2}, l\right)$-cross pattern in $(G, \Omega)$. As a technicality, in order to ensure that any pattern we inductively find will be a pattern in $(G, \Omega)$, we expand $G_{L}$ and $G_{R}$ to include a large planar swathe of $\bar{H}$ which will allow us to apply Theorem C.3. The only difficulty now will be to ensure that we did not have to delete too many vertices to obtain the two inductive decompositions. This will require a little more work and specifically considering large leap patterns as well.

Proof. The proof proceeds by induction on $|V(G)|$. We first fix the following values. Let $w$ be the function in Theorem C.1. Fix $k=\max \left\{k_{1}, k_{2}, k_{3}\right\}$. Let $T$ be defined to be

$$
T=2\left(\left(\begin{array}{l}
k \\
2
\end{array}\right)(l+1) k+2 k\right)+2 w\left(\left(\begin{array}{l}
k \\
2
\end{array}\right)(l+1) k+2 k\right)+1
$$

We assume that

$$
t=4\left[2 T+4 k^{2}+(2 k-1)+l+1\right] 4 k^{2} .
$$

The value $T$ is obtained by taking the bound on the order of a $(k, k(l+1))$-cross or leap pattern from Observation 1 and applying the insulation bound given by Theorem C.3.

The value of $\alpha$ will be determined by several recursive relations which arise in the proof. For now, we observe that if $k_{1}=2$ or $k_{2}=2$, then the theorem follows immediately from Theorem A.4. Thus, the theorem is true with $\alpha\left(2, k_{2}^{\prime}, k_{3}^{\prime}, l^{\prime}\right)=0$ for all $k_{2}^{\prime} \geq 2, k_{3}^{\prime} \geq 0$, and $l^{\prime} \geq 2$. Similarly, we see that the theorem is true with $\alpha\left(k_{1}^{\prime}, 2, k_{3}^{\prime}, l^{\prime}\right)=0$ again for all $k_{2}^{\prime} \geq 2, k_{3}^{\prime} \geq 0$, and $l^{\prime} \geq 2$. Finally, if we assume that a $(0, l)$-leap pattern is simply the empty pattern for all $l \geq 2$, then we see that the statement is trivial with $\alpha\left(k_{1}^{\prime}, k_{2}^{\prime}, 0, l^{\prime}\right)$ for all $k_{1}^{\prime} \geq 2, k_{2}^{\prime} \geq 2$, and $l^{\prime} \geq 2$. These observations will effectively serve as the boundary conditions for our recursive relation which determines $\alpha$. Finally, as a first step we assume

$$
\alpha\left(k_{1}, k_{2}, k_{3}, l\right) \geq\left(t 4 k^{2}\right)^{2}
$$

which will allow us to apply Lemma D.3.

We apply Lemma D.3. We can assume that there exists a set $Z \subseteq V(G)$ and a decomposition of $G_{L}, H, G_{R}$ of $G-Z$ satisfying outcome 3 . Let $S_{1}, S_{2}, S_{L}, S_{R}, H_{0}, \ldots, H_{n}, L_{1}, \ldots, L_{t}, x_{1}, \ldots, x_{t}, y_{1}, \ldots, y_{t}$ be as defined in the outcome. We will refer to the linkage $L_{1} \cup \cdots \cup L_{t}$ as $\mathcal{L}$. For each $L_{i}, 1 \leq i \leq t$, the vertices of $V\left(L_{i}\right) \cap V\left(H_{0}\right)$ split $G-Z$ into two pieces, one containing $G_{L}$ and one containing $G_{R}$. For all $1 \leq i \leq t$, we let $\left(A_{i}, B_{i}\right)$ be the separation $G$ with $A_{i} \cap B_{i}=Z \cup\left(V\left(L_{i}\right) \cap V\left(H_{0}\right)\right)$ such that $V\left(G_{L}\right) \cup Z \subseteq A_{i}$ and $V\left(G_{R}\right) \cup Z \subseteq B_{i}$ and the position of the vertices of $H$ determined by a curve in the disc through the vertices of $V\left(L_{i}\right) \cap V\left(H_{0}\right)$ following the path $L_{i}$. Note that, as described, the separation $\left(A_{i}, B_{i}\right)$ may not be uniquely defined. There might exist $H_{j}$ attaching to at most two vertices of $V\left(L_{i}\right) \cap V\left(H_{0}\right)$, in which case the curve could be shifted to include such $V\left(H_{j}\right)$ in either $A_{i}$ or $B_{i}$. In this case, we arbitrarily include such $V\left(H_{j}\right)$ in either $A_{i}$ or $B_{i}$. 
We quickly consider a couple of properties of the separations $\left(A_{i}, B_{i}\right)$. First, for all $i<j$, we have the property that $A_{i} \subseteq A_{j}$ and $B_{i} \supseteq B_{j}$. If we look at $A_{i} \cap B_{i}$, this is exactly the set of vertices of $L_{i}$ contained in $V\left(H_{0}\right)$ together with the set $Z$ of vertices. Finally, we have chosen the separations such that for $i<j$, the subgraph induced on $\left(B_{i} \cap A_{j}\right) \backslash Z$ is essentially the subgraph of $H_{0}$ "between" the paths $L_{i}$ and $L_{j}$, along with the small vortices $H_{r}$ with all attachments contained in that planar strip.

For every $i, 1 \leq i \leq t$, let $\operatorname{Ord}_{i}^{L}$ (respectively $\operatorname{Ord}_{i}^{R}$ ) be the cyclic order of $\left(S_{L} \backslash Z\right) \cup x_{1} S_{1} x_{i} \cup\left(V\left(L_{i}\right) \cap\right.$ $\left.V\left(H_{0}\right)\right) \cup y_{i} S_{w} y_{1}\left(\left(x_{t-i} S_{1} x_{t} \cup\left(S_{R} \backslash Z\right) \cup y_{t} S_{2} y_{i} \cup\left(V\left(L_{t}\right) \cap V\left(H_{0}\right)\right)\right.\right.$, respectively for $\left.\operatorname{Ord}_{i}^{R}\right)$ where the order is given by the order of $\Omega$ and the natural linear order of $V\left(L_{i}\right)$. Let $F_{i}^{L}$ be the subgraph $G\left[A_{i}\right]-Z$ and let $F_{i}^{R}$ be the subgraph $G\left[B_{i}\right]-Z$. Consider the societies $\left(F_{i}^{R}, \operatorname{Ord}_{i}^{R}\right)$ and $\left(F_{i}^{L}, \operatorname{Ord}_{i}^{L}\right)$ for $T \leq i \leq t-T$. The segment $V\left(L_{i}\right) \cap V\left(J_{0}\right)$ in $\left(F_{i}^{L}, \operatorname{Ord}_{i}^{L}\right)$ is $(T-1)$-insulated, and, similarly, the segment $V\left(L_{i}\right) \cap V\left(J_{0}\right)$ is $(T-1)$-insulated in $\left(F_{i}^{R}, \operatorname{Ord}_{i}^{R}\right)$. It follows by our choice of $T$, Observation 1, and Theorem C.3 that neither $\left(F_{i}^{R}, \operatorname{Ord}_{i}^{R}\right)$ nor $\left(F_{i}^{L}, \operatorname{Ord}_{i}^{L}\right)$ contains either a $k_{1}$-crosscap, a $\left(k_{2}, l\right)$-cross pattern, nor a $\left(k_{3}, l\right)$-leap pattern for all $T \leq i \leq t-T$.

For $1 \leq i \leq t$, we let $\tau_{i}$ be the maximal integer such that $\left(F_{i}^{L}, \operatorname{Ord}_{i}^{L}\right)$ contains a $\left(\tau_{i}, l\right)$-cross pattern. Similarly, we let $\gamma_{i}$ be the maximal integer such that $\left(F_{i}^{L}, \operatorname{Ord}_{i}^{L}\right)$ contains a $\left(\gamma_{i}, l\right)$-cross pattern. As we have just observed, $\tau_{i}<k_{2}$ for $i \geq T$ and $\gamma_{i} \leq k_{2}$ for $i \leq t-T$.

Claim D.5 For all $T \leq i \leq j \leq t-T$ with $|i-j| \geq l+1$, we may assume that $\tau_{i}+\gamma_{j}=k_{2}-1$ and either $\tau_{i}$ or $\gamma_{j}$ is equal to zero.

Proof. Fix the indices $i$ and $j$ satisfying the inequalities. We first will show that if $\tau_{i}+\gamma_{j} \geq k_{2}$, the theorem is proven. Let $\mathcal{P}_{i}$ be an $\left(\tau_{i}, l\right)$-cross pattern in $\left(F_{i}^{L}, O r d_{i}^{L}\right)$ and let $\mathcal{P}_{j}$ be a $\left(\gamma_{i}, l\right)$-cross pattern in $\left(F_{j}^{R}, \operatorname{Ord}_{j}^{R}\right)$. By Theorem C.3, the fact that $\tau_{i}<k_{2}$ and $\gamma_{i}<k_{2}$, and by our choice of $T$, we may assume that each of $\mathcal{P}_{i}$ and $\mathcal{P}_{j}$ has all it's endpoints contained in $\Omega$, i.e. both $\mathcal{P}_{i}$ and $\mathcal{P}_{j}$ are a $\left(\tau_{i}, l\right)$ and $\left(\gamma_{j}, l\right)$-cross patterns in $(G, \Omega)$, respectively. It follows that $\mathcal{P}_{i} \cup \mathcal{P}_{j} \cup \bigcup_{i^{\prime}=1}^{l} L_{i+i^{\prime}}$ forms a $\left(\tau_{i}+\gamma_{j}, l\right)$-cross pattern in $(G, \Omega)$, satisfying the theorem.

We conclude that $\tau_{i}+\gamma_{j}<k_{2}$. Assume, as a case, that both $\tau_{i} \leq k_{2}-2, \gamma_{j} \leq k_{2}-2$. It follows that $\left(F_{i}^{L}, \operatorname{Ord}_{i}^{L}\right)$ (and similarly, $\left(F_{j}^{R}, O r d_{j}^{R}\right)$ ) does not contain a $k_{1}$-crosscap, a $\left(k_{2}-1, l\right)$-cross pattern, nor a $\left(k_{3}, l\right)$-leap pattern. By induction and our assumption that $\left(F_{i}^{L}, \operatorname{Ord}_{i}^{L}\right)$ does not contain a $\left(\tau_{i}+1, l\right)$-cross pattern, we conclude that there exist a set $Z_{i}$ such that $\left(F_{i}^{L}-Z_{i}, O r d_{j}^{L}\right)$ has an $\alpha\left(k_{1}, k_{2}-1, k_{3}, l\right)$-bounded $\tau_{i}$-nearly flat decomposition. Similarly, there exists a subset $Z_{j}$ such that $\left(F_{j}^{R}-Z_{j}, \operatorname{Ord}_{j}^{R}\right)$ has an $\alpha\left(k_{1}, k_{2}-1, k_{3}, l\right)$ bounded $\gamma_{j}$-nearly flat decomposition. It follows that $\left(G-\left(Z \cup Z_{1} \cup Z_{2}\right), \Omega\right)$ has a $\left(2 \alpha\left(k_{1}, k_{2}-1, k_{3}, l\right)+|Z|\right)$ bounded $\left(\tau_{i}+\gamma_{j}\right)$-nearly flat decomposition. Recall that $|Z| \leq 4 k^{2}$. If we pick $\alpha\left(k_{1}, k_{2}, k_{3}, l\right)$ to satisfy the recursion relation

$$
\alpha\left(k_{1}, k_{2}, k_{3}, l\right) \geq 2 \alpha\left(k_{1}, k_{2}-1, k_{3}, l\right)+4 k^{2} \geq|Z|+\left|Z_{i}\right|+\left|Z_{j}\right|
$$

we see that the theorem holds.

It follows that we may assume that either $\tau_{i}$ or $\gamma_{j}$ is at least $k_{2}-1$. Putting the inequalities together, it follows that exactly one of $\tau_{i}$ or $\gamma_{j}$ is equal to $k_{2}-1$ and the other is then equal to zero. This proves the claim.

For the remainder of the proof, we assume without loss of generality, that $\gamma_{t-T}=k_{2}-1$ and that $\left(F_{i}^{R}, \operatorname{Ord}_{i}^{R}\right)$ has a 0 -nearly flat decomposition for all $T \leq i \leq t-T-l-1$. Then there exists a $\left(k_{2}-1, l\right)$-cross pattern $\mathcal{P}$ for $(G, \Omega)$ contained in $\left(F_{t-T}^{L}, \operatorname{Ord}_{t-T}^{L}\right)$. Fix this pattern $\mathcal{P}$ for the remainder of the proof.

Claim D.6 There exists an index $\bar{i}$ such that the subgraph $H\left[B_{\bar{i}} \cap A_{\bar{i}+2 T+4 k^{2}+l+1}\right]$ does not contain any neighbor of a vertex in $Z$.

Proof. For all $i, T \leq i \leq t-T-l-1$, if there existed a pair of crossing $\Omega$-paths $Q_{1}, Q_{2}$ contained in $G\left[A_{i}\right]$, it would follow that $\mathcal{P} \cup \bigcup_{j=1}^{l} L_{t-T-j} \cup Q_{1} \cup Q_{2}$ would form a $\left(k_{2}, l\right)$-cross pattern in $(G, \Omega)$. Given that 
$H$ satisfies d. It follows that for every $z \in Z$, the neighbors of $z$ in $H$ are limited; specifically, there exist indices $i(z)$ and $j(z)$ with $i(z)<j(z)$ and $j(z)-i(z)<4$ such that every neighbor of $z$ in $H$ is contained in $B_{i(z)} \cap A_{j(z)}$. We conclude (by the choice of $t$ ) that that there exists an index $\bar{i}$ as desired by the claim.

The remainder of the proof will split into two cases. If there does not exist a path from in $G-H^{\prime}$ from $S_{L}$ to $S_{R}$, then we inductively find the desired decomposition in each half of $G-H^{\prime}$ and glue the 3 decompositions together. Alternatively, if there does exist such a path, call it $P$, we find a $\left(k_{3}-1, l^{\prime}\right)$-leap pattern in a supergraph of $F_{i+2 T+4 k^{2}+l+1}^{L}$ for some suitable $l^{\prime}>l$, and extend it to a $\left(k_{3}, l\right)$-leap pattern using the path $P$.

Before doing so, we will need some more notation. Fix the indices $a=\bar{i}+T+4 k^{2}$ and $b=\bar{i}+T+4 k^{2}+l+1$. Fix $\overline{F^{L}}$ to be $G\left[A_{a}\right]-\left(Z \cap S_{R}\right)$ and $\overline{O r d^{L}}$ the cyclic order on the segment $S_{L} \cup s_{1} S_{1} s_{a} \cup\left(V\left(L_{a}\right) \cap V\left(H_{0}\right)\right) \cup t_{a} S_{2} t_{1}$ with the ordering taken from the order $\Omega$ and the natural ordering of the vertices of $L_{a}$. We similarly fix $\overline{F^{R}}$ to be $G\left[B_{b}\right]-\left(Z \cap S_{L}\right)$ and $\overline{O r d^{R}}$ the cyclic order on the segment $S_{R} \cup s_{b} S_{1} s_{t} \cup\left(V\left(L_{b}\right) \cap V\left(H_{0}\right)\right) \cup t_{t} S_{2} t_{b}$. We let $\bar{H}$ be the subgraph $G\left[B_{a} \cap A_{b}\right]-Z$. We let $\overline{O r d}$ be the cyclic order $V(\bar{H}) \cap\left(S_{1} \cup S_{2} \cup V\left(\overline{F^{R}}\right) \cup V\left(\overline{F^{L}}\right)\right)$ with the order taken from $\Omega$ and the natural linear order of the vertices $L_{a}$ and $L_{b}$. By construction, we know that $(\bar{H}, \overline{O r d})$ has a 0 -near flat decomposition. Also, we know that the vertices of $L_{a}$ contained in $\overline{F^{L}}$ are $T+4 k^{2}$-insulated in $\left(\overline{F^{L}}, \overline{O r d^{L}}\right)$. Similarly, the vertices of $L_{b}$ in $\overline{F^{R}}$ are $T+2 k-1$-insulated in $\left(\overline{F^{R}}, \overline{O r d^{R}}\right)$.

We first consider the subgraph $\overline{F^{L}}$, and let $(C, D)$ be a separation of minimal order with $\left(S_{L} \cup s_{1} S_{1} s_{a} \cup\right.$ $\left.t_{a} S_{2} t_{1}\right) \subseteq C$ and $Z \subseteq D$. We allow $(C, D)$ to be a trivial separation with $D=Z$, and so such a separation is guaranteed to exist and has order at most $|Z| \leq 4 k^{2}$. Let $Z^{\prime}=C \cap D$. Observe that for every vertex in $x \in D$, there exists an $x-Z$ path with no internal vertex in $\Omega$. Also, by the fact that $(C, D)$ is a separation of minimal order, for every $z \in Z^{\prime}$, there exists a $z-\Omega$ path in $G[C]$.

Claim D.7 $D$ does not contain any vertex in $A_{a} \cap B_{\bar{i}+4 k^{2}}$.

Proof. To see the claim, assume $x \in A_{a} \cap B_{\bar{i}+4 k^{2}}$ is such a vertex. Note that any path from $x$ to $Z$ in $\overline{F^{R}}$ must intersect the $4 k^{2}$ distinct paths $L_{j}$ for $\bar{i} \leq j \leq \bar{i}+4 k^{2}-1$. This follows from the near planarity of $H$ as well as the fact that no vertex of $Z$ has a neighbor in $A_{a} \cap B_{b}$. However, at most $2 k^{2}$ distinct paths $L_{j}$ intersect $D$. Thus no such $x$ exists, proving the claim.

An immediate consequence of this fact is that $V\left(L_{a}\right) \cap V\left(H_{0}\right)$ is $T$-insulated in $\overline{F^{L}}[C]$.

Now consider the graph $\overline{F^{R}} \cup G[D]$.

Claim D.8 There exists a $Z^{\prime}-\left(s_{b} S_{1} s_{t} \cup S_{R} \cup t_{t} S_{2} t_{b}\right)$ path in $\overline{F^{R}} \cup G[D]$.

Proof. Assume, that such a path does not exist. Then there exists a separation $\left(C^{\prime}, D^{\prime}\right)$ of $\overline{F^{R}} \cup G[D]$ of order zero with $Z^{\prime} \subseteq C^{\prime}$ and $s_{b} S_{1} s_{t} \cup S_{R} \cup t_{t} S_{2} t_{b} \subseteq D^{\prime}$.

If we now look at this separation, we see that $G=\overline{F^{L}}[C] \cup G\left[C^{\prime}\right] \cup \bar{H} \cup G\left[D^{\prime}\right]-E(\bar{H})$. Moreover, these subgraphs are edge disjoint.

Recall that $F_{t-T}^{R}$ contained a $\left(k_{2}-1, l\right)$-pattern $\mathcal{P}$ with endpoints in $\Omega$. The pattern $\mathcal{P}$ is also contained in $G\left[D^{\prime}\right]$. Also note that $V\left(L_{a}\right) \cap V\left(H_{0}\right)$ is highly insulated in $\overline{F^{L}}[C]$ and that $D$ does not contain any vertex of $A_{a} \cap B_{\bar{i}+4 k^{2}}$. Thus $V\left(L_{a}\right) \cap V\left(H_{0}\right)$ is $T$ insulated in $\bar{F}^{L}[C] \cup G\left[C^{\prime}\right]$ as well. We conclude that if $\left(\overline{F^{L}}[C] \cup G\left[C^{\prime}\right], \overline{\operatorname{Ord}^{L}}\right)$ contains a pair of crossing paths, we may assume that they have endpoints in $\Omega$. Combining the pair of crossing paths with $\mathcal{P}$ and the paths $L_{a+1}, \ldots, L_{a+l}$, we see that $(G, \Omega)$ contains a $\left(k_{2}, l\right)$-cross pattern. 
We conclude that $\left(\overline{F^{L}}[C] \cup G\left[C^{\prime}\right], \overline{O r d^{L}}\right)$ has a 0-nearly flat decomposition. We now turn our attention to $G\left[D^{\prime}\right]$. The set $C^{\prime}$ cannot contain any vertex of $B_{\bar{i}+2 T+4 k^{2}+(2 k-1)+1} \cap A_{b}$, as this would imply the existence of a path from $\overline{O r d^{R}}$ to $\overline{O r d^{L}}$ avoiding both $Z$ and $V(\bar{H})$, a contradiction. It follows that set $V\left(L_{b}\right) \cap V\left(\overline{F^{R}}\right)$ is $T$-insulated in $\left(G\left[D^{\prime}\right], \overline{O r d^{R}}\right)$. Thus, if $\left(\left[G\left[D^{\prime}\right], \overline{O r d^{R}}\right)\right.$ has either a $k_{1}$-crosscap, a $\left(k_{2}, l\right)$-cross pattern, or a $\left(k_{3}, l\right)$-leap pattern, the theorem is proven. By induction, we conclude that there exists a set $X \subseteq D^{\prime}$ with $|X| \leq \alpha\left(k_{1}, k_{2}, k_{3}, l\right)$ and an $\alpha\left(k_{1}, k_{2}, k_{3}, l\right)$-bounded $\left(k_{2}-1\right)$-nearly flat decomposition of $\left(G\left[D^{\prime}\right]-\right.$ $\left.X, \overline{O r d} d^{R}-X\right)$. Combined with the 0-nearly flat decompositions for $(\bar{H}, \overline{O r d})$ and $\left(\overline{F^{L}}[C] \cup G\left[C^{\prime}\right], \overline{\operatorname{Ord}^{L}}\right)$ yields an $\alpha\left(k_{1}, k_{2}, k_{3}, l\right)$-bounded $\left(k_{2}-1\right)$-nearly flat decomposition of $(G-X, \Omega)$. This completes the proof of the claim.

We now define the separation $\left(C^{\prime \prime}, D^{\prime \prime}\right)$ of $\overline{F^{R}} \cup G[D]$ as follows. If there exist $2 k-1$ disjoint paths from $Z^{\prime}$ to $\left(s_{b} S_{1} s_{t} \cup S_{R} \cup t_{t} S_{2} t_{b}\right)$, we define $\left(C^{\prime \prime}, D^{\prime \prime}\right)$ to be the trivial separation with $C^{\prime \prime}=Z^{\prime}$ and $D^{\prime \prime}=V\left(\overline{F^{R}}\right) \cup D$. If such $2 k-1$ disjoint paths do not exist, we now apply Lemma D.2 to the subgraph $\overline{F^{R}} \cup G[D]$ and the two sets $Z^{\prime}$ and $\left(s_{b} S_{1} s_{t} \cup S_{R} \cup t_{t} S_{2} t_{b}\right)$ with the value $2 k-1$ to find the separation $\left(C^{\prime \prime}, D^{\prime \prime}\right)$.

By induction, we see that the society $\left(\left(\overline{F^{R}} \cup G[D]\right)\left[D^{\prime \prime}\right], \overline{\text { Ord }}^{L}-C^{\prime \prime}\right)$ contains a $\left(k_{3}-1, k(l+1)\right)$-leap pattern or the desired decomposition.

Claim D.9 There does not exist a set $X \subseteq V\left(\overline{F^{R}}\right) \cup D$ with $|X| \leq \alpha\left(k_{1}, k_{2}, k_{3}-1, k(l+1)\right)$ such that the society $\left(\left(\overline{F^{R}} \cup G[D]\right)-\left(\left(C^{\prime \prime} \cap D^{\prime \prime}\right) \cup X\right), \overline{\text { Ord }}^{L}-\left(\left(C^{\prime \prime} \cap D^{\prime \prime}\right) \cup\right)\right)$ contains an $\alpha\left(k_{1}, k_{2}, k_{3}-1, k(l+1)\right)$ bounded $\left(k_{2}-1\right)$-nearly flat decomposition.

Proof. Assume, to reach a contradiction, that such a set $X$ and decomposition exist. We can merge the 0-nearly flat decompositions for $\left(\overline{F^{L}} \cup G\left[C^{\prime}\right], \overline{O r d^{L}}\right),(\bar{H}, \overline{O r d})$, and the $\left(k_{2}-1\right)$-nearly flat decomposition of $\left(\left(\overline{F^{R}} \cup G[D]\right)-\left(\left(C^{\prime \prime} \cap D^{\prime \prime}\right) \cup X\right), \overline{O r d^{L}}-\left(\left(C^{\prime \prime} \cap D^{\prime \prime}\right) \cup X\right)\right)$ to find an $\alpha\left(k_{1}, k_{2}, k_{3}-1, k(l+1)\right)$-bounded $\left(k_{2}-1\right)$-nearly flat embedding of $\left(G-\left((C \cap D) \cup\left(C^{\prime \prime} \cap D^{\prime \prime}\right) \cup X\right), \Omega-\left((C \cap D) \cup\left(C^{\prime \prime} \cap D^{\prime \prime}\right) \cup X\right)\right)$. The theorem now follows if we assume that

$$
\alpha\left(k_{1}, k_{2}, k_{3}, l\right) \geq \alpha\left(k_{1}, k_{2}, k_{3}-1, k(l+1)\right)+4 k^{2}+(2 k-1) .
$$

This proves the claim.

We see, by induction and the previous claim, that there exists a $\left(k_{3}-1, k(l+1)\right)$-leap pattern in the society $\left(\left(\overline{F^{R}} \cup G[D]\right)\left[D^{\prime \prime}\right]-\left(C^{\prime \prime} \cap D^{\prime \prime}\right), \overline{O r d^{L}}-\left(C^{\prime \prime} \cap D^{\prime \prime}\right)\right)$. Fix $\overline{\mathcal{P}}$ to be such a pattern.

Claim D.10 $C^{\prime \prime}$ does not contain any vertices of $\overline{\operatorname{Ord}^{L}}$, and consequently, $\left|C^{\prime \prime} \cap D^{\prime \prime}\right|=2 k-1$.

Proof. Assume $C^{\prime \prime}$ contains a vertex of $\overline{\operatorname{Ord}^{L}}$. Then there exists a path in $G\left[C^{\prime \prime}\right]$ linking a vertex of $\overline{\operatorname{Ord}} \mathrm{C}^{L}$ to $Z^{\prime}$. Then in $\overline{F^{L}}[C]$, there exists a path linking that vertex to a vertex of $\overline{O r d^{L}}$. Thus, there exists a path $P$ linking in $\overline{F^{L}}[C] \cup G\left[C^{\prime \prime}\right]$ with one end in $\overline{\operatorname{Ord}^{L}}$ and the other end in $\overline{O r d^{R}}$. Moreover, the path $P$ is disjoint from $\overline{\mathcal{P}}$ by construction. It follows that $P \cup \overline{\mathcal{P}} \cup L_{a} \cup \cdots \cup L_{a+l-1}$ contains a $\left(k_{3}, l\right)$-leap pattern, as desired.

Thus, $C^{\prime \prime}$ does not contain any vertices of $\overline{\operatorname{Ord}^{L}}$, and the second half of the claim now follows trivially from the outcome of Lemma D.2.

We are now ready to complete the proof of the theorem. Let $\overline{\mathcal{P}}$ consist of two disjoint subpatterns, $\overline{\mathcal{R}}$ and $\overline{\mathcal{Q}}$ where $\overline{\mathcal{R}}$ consists of pairwise non-crossing $\overline{\operatorname{Ord}^{L}}$-paths and $\overline{\mathcal{Q}}$ consists of $k_{3}-1$ pairwise disjoint paths whose endpoints are at dist $\overline{\mathcal{R}}$ at least $k(l+1)$. We let $\overline{\mathcal{S}}$ be a linkage of order $2 k-1$ in $\overline{F^{R}}\left[D^{\prime \prime}\right]$ each with one endpoint in $C^{\prime \prime} \cap D^{\prime \prime}$ and the other endpoint in $\overline{\operatorname{Ord}^{R}} \cup V(\overline{\mathcal{R}})$. Note that the linkage $\bar{S}$ is guaranteed to exist by Lemma D.2 and the previous claim. 
We consider an auxiliary graph $A$ as follows. For all $S \in \overline{\mathcal{S}}$, let $x_{S}$ be the first vertex of $S$ contained in $\overline{O r d^{R}} \cup V(\overline{\mathcal{R}})$ when traversing $S$ from $C^{\prime \prime} \cap D^{\prime \prime}$ to $\overline{O r d^{R}}$ and let $S x_{S}$ be the subpath of $S$ with one end $x_{S}$ and the other end in $C^{\prime \prime} \cap D^{\prime \prime}$. The vertex set of $A$ is the set $V(\overline{\mathcal{Q}}) \cup\left\{V\left(S x_{S}\right) \mid S \in \overline{\mathcal{S}}\right\} \cup\{v\}$ and edge set $E(\overline{\mathcal{Q}}) \cup\left\{E\left(S x_{S}\right) \mid S \in \overline{\mathcal{S}}\right\}$ as well as an edge from $v$ to every vertex of $C^{\prime \prime} \cap D^{\prime \prime}$. We fix the subset $X_{A} \subseteq V(A)$ to be the vertices $\{v\} \cup\left\{x_{S} \mid S \in \overline{\mathcal{S}}\right\} \cup\left(\overline{\operatorname{Ord}^{R}} \cap V(\overline{\mathcal{Q}})\right)$ and define a distance function $d_{A}$ on $X_{a}$ so that $d_{A}(v, x)=l$ for all $x \in X_{A}, x \neq v$, and $d_{A}(x, y)=\operatorname{dist}_{\overline{\mathcal{R}}}(x, y)$ for all $x, y \in X_{A}, x \neq v, y \neq v$. We see that $\overline{\mathcal{Q}}$ forms a set of $k-1$ pairwise disjoint $X_{A}$-paths whose endpoints are at distance $k(l+1)$ with respect to the distance function $d_{A}$, and $v S x_{S}$ form $2 k-1$ internally disjoint paths from $v$ to distinct vertices of $X_{A}$.

We apply Lemma D.2 to the auxiliary graph $A$ with the $X_{A}$-paths. We conclude that there exist pairwise disjoint $X_{A}$-paths $Q_{1}, \ldots, Q_{k}$ with endpoints contained in $\left(\overline{\operatorname{Ord}^{R}} \cap V(\overline{\mathcal{Q}})\right) \cup\left\{v, v^{\prime}\right\}$ for some vertex $v^{\prime} \in X_{A}$ such that for all $1 \leq i \leq k$, the endpoints of $Q_{i}$ are at distance at least $l+1$ with the distance function $d_{A}$. We will convert the paths $Q_{i}, 1 \leq i \leq k$ into $\Omega$-paths as follows. Let $i$ be such that $Q_{i}$ has $v$ as an endpoint, and $i^{\prime}$ such that $Q_{i^{\prime}}$ has $v^{\prime}$ as an endpoint (it is possible that $i=i^{\prime}$ ). We let $M_{1}$ be a path in $G\left[C^{\prime \prime}\right] \cup \overline{F^{L}}[C]$ linking $Q_{i} \cap\left(C^{\prime \prime} \cap D^{\prime \prime}\right)$ to $\overline{O r d^{L}}$. In the case that $v^{\prime} \in \overline{O r d^{R}}$, we let $M_{2}$ be the trivial path $\left\{v^{\prime}\right\}$ and define $R$ to be the empty path. If $v^{\prime} \in V(R)$ for some $R \in \overline{\mathcal{R}}$, we let $M_{2}$ be a subpath of $R$ linking $v^{\prime}$ and $\overline{O r d} d^{R}$. We now let $Q_{i}^{\prime}=\left(Q_{i}-v\right) \cup M_{1}$ and $Q_{i^{\prime}}^{\prime}=Q_{i^{\prime}} \cup M_{2}$ (and in the case $\left.i=i^{\prime}, Q_{i}^{\prime}=\left(Q_{i}-v\right) \cup M_{1} \cup M_{2}\right)$. We let $Q_{j}^{\prime}=Q_{j}$ for all $j \notin\left\{i, i^{\prime}\right\}$. We conclude that $Q_{1}^{\prime} \cup \ldots Q_{k}^{\prime} \cup L_{a} \cup L_{a+1} \cup \cdots \cup L_{a+l} \cup(\overline{\mathcal{R}}-R)$ forms a $\left(k_{3}, l\right)$-leap pattern, completing the proof of the theorem.

\section{E Embedding a society into a crosscap or handle}

Before stating the main result of this section, we need the following definitions. We say $\Omega$-paths $P_{1}, \ldots, P_{k}$ in a society $(G, \Omega)$ are independent if they are pairwise disjoint and there exist disjoint segments $S_{1}, \ldots, S_{k}$ of $\Omega$ such that the endpoints of $P_{i}$ are contained in $S_{i}$ for all $1 \leq i \leq k$.

For our main theorem in this section, we will need to allow for one more possible pattern as an outcome.

Let $(G, \Omega)$ be a society, and let $k$ and $l$ be positive integers and let $\mathcal{P}$ be a pattern in $(G, \Omega)$. We say that $\mathcal{P}$ is a twisted $(k, l)$-cross pattern if there exists a segment $S$ of $\Omega$ such that the cyclic order $\Omega^{\prime}$ obtained from $\Omega$ by reversing the order of the segment $S$ satisfies the property that $\mathcal{P}$ is a $(k, l)$-cross pattern in the society $\left(G, \Omega^{\prime}\right)$.

We are now ready to state the main theorem of this section. This is the more exact statement of Theorem 2.3 , which follows as an immediate consequence of the stronger statement.

Theorem E.1 For all positive integers $t, k$, and $l$, there exist values $f(t, k)$ and $a(k, l)$ satisfying the following. Let $(G, \Omega)$ be a society, and let $\mathcal{M}$ be either a $f(t, k)$-crosscap or $f(t, k)$-handle in $G$. Assume there exists a cycle $C$ such that $V(C)=\Omega$ and the natural cyclic order of $V(C)$ associated with the cycle is the same as $\Omega$. Then one of the following outcomes holds.

1. There exists a $(k, l)$-cross pattern or a $(k, l)$-twisted cross pattern.

2. There exists a segment $S$ of $\Omega$ such that if we let $\Omega^{\prime}$ be the cyclic order of $\Omega \backslash S$ induced by $\Omega$, then there exists a pattern $\mathcal{P} \cup \mathcal{Q}$ in $\left(G, \Omega^{\prime}\right)$ such that $\mathcal{P}$ consists of $k$ independent $\Omega$-paths $P_{1}, \ldots, P_{k}$ and for every $P_{i}$, there exist $l$ distinct components of $\mathcal{Q}$ which cross $P_{i}$.

3. Let $\Sigma_{1}$ and $\Sigma_{2}$ be the surfaces comprised of the disc plus a single crosscap or a single handle, respectively. There exists a subset $Z \subseteq V(G)$ with $|Z| \leq a(k, l)$ such that $(G-Z, \Omega-Z)$ has a 1-near embedding $H_{0}, H_{1}, \ldots, H_{m}$ in $\Sigma_{1}$ if $M$ is a crosscap, and in $\Sigma_{2}$ in the case $M$ is a handle such that:

$i$. the vertices embedded in the boundary of the disc are exactly the vertices of $\Omega-Z$, and they are embedded in the correct cyclic order; 
ii. there exists a subgraph $M^{\prime}$ of $M$ forming a $t$-crosscap or $t$-handle in $H_{0} \cup \bigcup_{i=1}^{m} H_{i}$.

In order to prove Theorem E.1, we need some further notation and auxiliary results. Let $(G, \Omega)$ be a society and let $\mathcal{M}$ be a $k$-crosscap or $k$-handle for some positive integer $k$. In this section, we consider the embedding $\sigma$ of $(\mathcal{M} \cup \Omega, \Omega)$ in the natural way into the disc plus a crosscap (or in the disc plus a handle in the case that $\mathcal{M}$ is a $k$-handle) such that the only vertices embedded onto the boundary of the disc are exactly the vertices $\Omega$ in the order specified by $\Omega$. This allows us to define a facial set of $\mathcal{M}$, which is a subset $Y \subseteq \Omega$ such that $Y$ is the set of $\Omega$ vertices contained in a single face of the embedding $\sigma$. Note that in this embedding $\sigma$, we do not regard the infinite region bounded by $\Omega$ as a "facial set". A facial cycle consists of all the vertices of $\mathcal{M} \cup \Omega$ contained in a single face. Assume that $F$ is a facial cycle. We say $F$ is dividing in $G$ if there does not exist an $\mathcal{M} \cup \Omega$-bridge with attachments in both $V(\mathcal{M}) \backslash F$ and in $V(F)$. The compass of $F$ is the subgraph formed by $F$ as well as every $\mathcal{M} \cup \Omega$-bridge with all attachments contained in $V(F)$.

We define the distance function $d_{\mathcal{M}}$ between two vertices $x$ and $y$ as minimal number of points in $S \cap \sigma(\Omega \cup \mathcal{M})$ for every curve $S$ in the surface linking $x$ and $y$. But we do not allow the curve $S$ to pass through the infinite region bounded by $\Omega$. Note that $d$ is always non-negative and trivially satisfies the triangle inequality. Also, the following observation follows immediately from the definition.

Observation 3 Let $(G, \Omega)$ be a society and $\mathcal{M}$ a crosscap or handle in $G$. If $\mathcal{M}^{\prime}$ is a sublinkage of $\mathcal{M}$ forming a crosscap or handle, then for all vertices $x$ and $y$ in $G, d_{\mathcal{M}^{\prime}}(x, y) \leq d_{\mathcal{M}}(x, y)$. Moreover, if $\mathcal{M}^{\prime}=\mathcal{M}-R$ for some component $R \in \mathcal{M}$, then $d_{\mathcal{M}}(x, y)-1 \leq d_{\mathcal{M}^{\prime}}(x, y) \leq d_{\mathcal{M}}(x, y)$.

Recall that a set of $X$-paths is $(l, t)$-spread for integers $l$ and $t$ if they satisfy $i$. and $i i$. in Lemma B.1. We need the following lemma.

Lemma E.2 There exist functions $f_{1}, f_{2}$, and $f_{3}$ satisfying the following. Let $k$, $t$, and $l$ be positive integers. Let $\mathcal{M}$ be either an $f_{1}(t, k)$-crosscap or an $f_{1}(t, k)$-handle in a society $(G, \Omega)$. Let $\mathcal{P}$ be an $f_{2}(k)$-linkage of $(\mathcal{M} \cup \Omega)$-paths which is $\left(f_{3}(l, k), f_{3}(l, k)\right)$-spread with respect to the distance function $d_{\mathcal{M}}$. Then there exist a sublinkage $\mathcal{M}^{\prime}$ of $\mathcal{M}$ and a pattern $\mathcal{P}^{\prime}$ of order $k$ such that the components of $\mathcal{P}^{\prime}$ and $\mathcal{M}^{\prime}$ are pairwise disjoint and the paths $\mathcal{P}^{\prime}$ are $(l, l)$-spread with respect to the distance function $d_{\mathcal{M}^{\prime}}$.

Proof. Let $k, t$, and $l$ be given. We prove the lemma with $f_{1}(t, k)=t+2 k+1, f_{2}(k)=4 k^{2}$, and $f_{3}(l, k)=l+2 k+1$. Assume, to reach a contradiction, that the desired patterns $\mathcal{P}^{\prime}$ and $\mathcal{M}^{\prime}$ do not exist.

First, we see that there does not exist a component $R \in \mathcal{M}$ such that at least $2 k$ components have an endpoint which is a vertex of $R \backslash \Omega$. Assume that $R$ is such a component and that $P_{1}, \ldots, P_{2 k}$ are distinct components of $\mathcal{P}$ with one endpoint in $R$. Label the endpoint of $P_{i}$ in $R$ as $x_{i}$, and assume that $x_{1}, x_{2}, \ldots, x_{2 k}$ occur on $R$ in that order. Since the vertices $x_{i}$ and $x_{j}$ are of distance $d_{\mathcal{M}}$ at most 1 , we see that the other endpoints of $P_{i}$ and $P_{j}$ must be of $d_{\mathcal{M}}$ at least $l+2 k+1$ since $\mathcal{P}$ is $\left(f_{3}(l, k), f_{3}(l, k)\right)$-spread with respect to the distance function $d_{\mathcal{M}}$. For $1 \leq i \leq 2 k$, we define paths $R_{i}$ as follows. If $P_{i}$ has its other endpoint contained in a component of $\mathcal{M}$ distinct from $R$, then we let $R_{i}$ be this component of $\mathcal{M}$. Otherwise, $P_{i}$ has its other endpoint in $\Omega$, and let $R_{i}$ be the endpoint of $P_{i}$ in $\Omega \backslash R$. Fix $\mathcal{M}^{\prime}$ to be $\mathcal{M}-\left(\bigcup_{i=1}^{2 k} R_{i} \cup R\right)$. By the Observation 3, for every pair of vertices $u$ and $v, d_{\mathcal{M}^{\prime}}(u, v) \geq d_{\mathcal{M}}(u, v)-(2 k+1) \geq l$. Moreover, for all $j, 1 \leq j \leq k$, we see that $R_{2 j} \cup P_{2 j} \cup P_{2 j+1} \cup R_{2 j+1}$ contains an $\Omega$-path, call it $P_{j}^{\prime}$, whose endpoints are of distance $d_{\mathcal{M}}$ at least $l+2 k+1$. The paths $P_{j}^{\prime}$ are disjoint from $\mathcal{M}^{\prime}$ and also pairwise disjoint, contradicting our choice of $G$ to form a counterexample.

We conclude that each path $P \in \mathcal{P}$ has endpoints in a common $R \in \mathcal{M}$ (including the ends of $R$ ) for at most $2 k-1$ other components of $\mathcal{M}$. By discarding a factor of at most $4 k$ of $\mathcal{P}$, we can conclude that there exists a subset $I \subseteq\left\{1, \ldots, 4 k^{2}\right\}$ of size $k$ such that for all $i, i^{\prime} \in I$, there does not exist $R \in \mathcal{M}$ such both $P_{i}$ and $P_{i^{\prime}}$ have endpoints in $R$.

For all $i \in I$, label the endpoints of $P_{i}$ as $x_{i}^{j}$ for $j=1,2$. For every $i \in I$ and $j=1,2$, define $R_{i}^{j}$ to be the component of $\mathcal{M}$ containing $x_{i}^{j}$ when such a component exists, and otherwise let $R_{i}^{j}$ be equal to $x_{i}^{j}$. 
For every $i \in I$, there exists an $\Omega$-path contained in $R_{i}^{1} \cup R_{i}^{2} \cup P_{i}$ with endpoints of distance $d_{\mathcal{M}}$ at least $l+2 k+1$. Fix such a path and call it $P_{i}^{\prime}$. We now see that $\mathcal{M}^{\prime}=\mathcal{M}-\left(\bigcup_{i \in I}\left(R_{i}^{1} \cup R_{i}^{2}\right)\right)$ along with the pattern $\mathcal{P}^{\prime}=P_{1}^{\prime} \cup \cdots \cup P_{k}^{\prime}$ satisfies the statement of the lemma, contradicting our choice of counterexample. This completes the proof of the lemma.

We are now ready to prove Theorem E.1.

Assume the theorem is false, and let $(G, \Omega)$ form a counterexample. Let $t, k, l$ be as in Theorem E.1. Let $k^{\prime}=k^{\prime}(k, l)$ be determined later. We assume that $f(t, k)$ in Theorem E. 1 is bigger than $f_{1}\left(t, k^{\prime}\right)$ in Lemma E.2, and $f(t, k)>(2 k+1)\left(l+2 f_{3}\left(3, k^{\prime}\right)\right) 2^{3 f_{2}\left(k^{\prime}\right)}$. Moreover, we assume $f_{3}\left(3, k^{\prime}\right) \geq t$ and $\alpha(k, l) \geq f_{2}\left(k^{\prime}\right)$, where $f_{2}, f_{3}$ come from Lemma E.2.

We first apply Lemma B.1 with $k=f_{2}\left(k^{\prime}\right)$ and $l=t=f_{3}\left(3, k^{\prime}\right)$ to $(G, \Omega)$ and $X=\mathcal{M}$, where $f_{2}, f_{3}$ come from Lemma E.2. Since there are two possibilities for the outcome of Lemma B.1, we now consider the two cases.

Case 1. There is an $f_{2}\left(k^{\prime}\right)$-linkage $\mathcal{P}$ of $(\mathcal{M} \cup \Omega)$-paths which is $\left(f_{3}\left(3, k^{\prime}\right), f_{3}\left(3, k^{\prime}\right)\right)$-spread with respect to the distance function $d_{\mathcal{M}}$.

We apply Lemma E.2 to the $f_{2}\left(k^{\prime}\right)$-linkage $\mathcal{P}$ and $\mathcal{M}$. Thus there exists a sublinkage $\mathcal{M}^{\prime}$ of $\mathcal{M}$ and a pattern $\mathcal{P}^{\prime}$ of order $k^{\prime}$ such that the components of $\mathcal{P}^{\prime}$ and $\mathcal{M}^{\prime}$ are pairwise disjoint and the paths $\mathcal{P}^{\prime}$ are $(3,3)$-spread with respect to the distance function $d_{\mathcal{M}^{\prime}}$.

The rest of the proof is almost identical to the proof given in Lemma 27 of [17].

We say that two components $P, P^{\prime} \in \mathcal{P}^{\prime}$ are facially incident if there exists a facial cycle of $\mathcal{M}^{\prime}$ that contains both an endvertex of $P$ and of $P^{\prime}$. We first prove the following claim.

Claim E.3 If there are $9\left(18(2 k l)^{2}((2 k l)+2)\right)^{3}$ components in $\mathcal{P}^{\prime}$ which are pairwise not facially incident, then there exist pairwise disjoint paths $E_{i}, F_{i}$ and segments $T_{i}$ for $1 \leq i \leq(2 k l)^{2}((2 k l)+2)$ with the following properties.

a. The segments $T_{i}$ are disjoint and occur in the order $T_{1}, \ldots, T_{(2 k l)^{2}((2 k l)+2)}$ in $\Omega$.

b. The disjoint paths $E_{i}$ and $F_{i}$ each have exactly one end in $T_{i}$ and are each disjoint from $T_{j}$ for all $1 \leq i, j \leq(2 k l)^{2}((2 k l)+2), j \neq i$.

c. The disjoint paths $E_{i}, 1 \leq i \leq(2 k l)^{2}((2 k l)+2)$, either form a crosscap or are pairwise non-crossing; the disjoint paths $F_{i}, 1 \leq i \leq(2 k l)^{2}((2 k l)+2)$, either form a crosscap or are pairwise non-crossing.

d. If the disjoint paths $E_{i}$ form a crosscap, then $E_{i}$ and $F_{i}$ do not cross for all $1 \leq i \leq(2 k l)^{2}((2 k l)+2)$; if the disjoint paths $E_{i}$ do not pairwise cross, then $E_{i}$ and $F_{i}$ do cross for all $1 \leq i \leq(2 k l)^{2}((2 k l)+2)$.

e. There exists a segment $T$ containing $T_{i}$, and exactly one endpoint of each path $E_{i}$ and $F_{i}$ for all $1 \leq i \leq(2 k l)^{2}((2 k l)+2)$.

Proof. Note that the statement of Claim E.3 is almost identical to Claim 29 in [17]. The statement follows immediately from Claim 29 in [17] by suppressing vertices of degree at most 2.

It was shown in [17] that given such $E_{i}, F_{i}$, and $T_{i}$ as in the conclusion of Claim E.3, either 1 or 2 in Theorem E.1 is satisfied.

Claim E.4 Let $E_{i}, F_{i}, T_{i}$, and T satisfy a. - e. as in the conclusion of Claim E.3 for $1 \leq i \leq(2 k l)^{2}((2 k l)+2)$. Then either $(G, \Omega)$ satisfies 1 in Theorem E.1, with the additional property that every path of the cross pattern or twisted cross pattern has one end in $T$ and one end in $\Omega \backslash T$, or $(G, \Omega)$ satisfies 2 in Theorem E.1, with the additional property that the segment $S$ satisfies $V(S) \subseteq \Omega \backslash T$. 
Let us observe that, as in the application of Claim 29 of [17], Claim 30 in [17] again only deals with the case that each $E_{i}$ and $F_{i}$ is a single edge. We can apply the result of the claim by suppressing vertices of degree at most 2, and conclude that Claim E.4 holds.

The following claim is a re-statement of Claim 28 in [17].

Claim E.5 If there is a facial set $C$ that contains at least $(k+4) 4 k^{2}$ endpoints of the components in $\mathcal{P}^{\prime}$, then 2 in Theorem E.1 holds.

Claims E.3, E.4, together with Claim E.5 imply that if we assume $k^{\prime} \geq 2(k+4) 4 k^{2} 9\left(18(2 k l)^{2}((2 k l)+2)\right)^{3}$, then the theorem holds. This completes the analysis of Case 1.

Case 2. There exist vertices $z_{1}, \ldots, z_{3 f_{2}\left(k^{\prime}\right)-3} \in V(G)$ and $Z \in V(G)$ with $|Z| \leq f_{2}\left(k^{\prime}\right)-1$ such that every $((\mathcal{M} \cup \Omega)-Z)$-path $P$ in $G-Z$ with endpoints $x$ and $y$ either satisfies $d_{(\mathcal{M} \cup \Omega)-Z}(x, y)<f_{3}\left(3, k^{\prime}\right)$ or $x, y \in \bigcup_{i=1}^{3 f_{2}\left(k^{\prime}\right)-3} B\left(z_{i}, f_{3}\left(3, k^{\prime}\right)\right)$.

We let $S_{1}, \ldots, S_{m}$ be the segments of $\Omega$ demarcated by the vertices of $\mathcal{M} \cap \Omega$. We observe that for any pair of indices $i$ and $j$, there exists a subgraph $\mathcal{M}^{\prime}$ of $\mathcal{M}$ such that $S_{i}$ and $S_{j}$ are contained in the same facial set of $\mathcal{M}^{\prime}$ and if $\mathcal{M}$ is a $m$-crosscap, then $\mathcal{M}^{\prime}$ is an $m / 2$-crosscap, and if $\mathcal{M}$ is a $m$-handle, then $\mathcal{M}^{\prime}$ is an $m / 2$ handle. Since there are at most $3 f_{2}\left(k^{\prime}\right)-3$ balls $B\left(z_{i}, f_{3}\left(3, k^{\prime}\right)\right)$ that cover all the long $((\mathcal{M} \cup \Omega)-Z)$-paths (i.e, a path with endpoints $x$ and $y$ such that the distance $d_{(\mathcal{M} \cup \Omega)-Z}(x, y) \geq f_{3}\left(3, k^{\prime}\right)$ ) in $G-Z$, thus by the above remark, there is a sublinkage $\mathcal{M}^{\prime \prime}$ of $\mathcal{M}$ in $G-Z$ such that $\mathcal{M}^{\prime \prime}$ is either a $m^{\prime}$-handle or a $m^{\prime}$-crosscap with $m^{\prime}=(2 k+1)\left(t+l+2 f_{3}\left(3, k^{\prime}\right)\right.$, and moreover, there is one special facial cycle of $\mathcal{M}^{\prime \prime}$ that contains all the balls $B\left(z_{i}, f_{3}\left(3, k^{\prime}\right)\right)$ (note that $\left.f(t, k)>(2 k+1)\left(l+2 f_{3}\left(3, k^{\prime}\right)\right) 2^{3 f_{2}\left(k^{\prime}\right)}\right)$.

Again, we let $S_{1}^{\prime}, \ldots, S_{m^{\prime \prime}}^{\prime}$ be the segments of $\Omega$ demarcated by the vertices of $\mathcal{M}^{\prime \prime} \cap \Omega$ so that $C\left[S_{i}^{\prime}\right]$, i.e., the facial cycle containing $S_{i}^{\prime}$, forms internally disjoint subpaths of $C$. We label the $S_{i}^{\prime}$ such that $S_{1}^{\prime}, \ldots, S_{m^{\prime \prime}}^{\prime}$ occur in that order of $\Omega$. If $\mathcal{M}^{\prime \prime}$ is a $m^{\prime}$-crosscap, note $m^{\prime \prime}=m^{\prime}$ and if $\mathcal{M}^{\prime \prime}$ is a $m^{\prime}$-handle, then $m^{\prime \prime}=2 m^{\prime}$. We also define $C_{j}\left[S_{i}^{\prime}\right]$ to be the union of $j$ facial cycles containing $S_{i}^{\prime} \cup \cdots \cup S_{i+j-1}^{\prime}$. We assume that the facial cycle containing $S_{m^{\prime \prime}}$ contains all the balls $B\left(z_{i}, f_{3}\left(3, k^{\prime}\right)\right)$.

We now prove the following claims;

Claim E.6 If there are $k$ indices $\pi(1), \ldots, \pi(k)$ with the following properties.

- $\pi(1) \leq \pi(2) \leq \ldots, \leq \pi(k)$.

- $\pi(i+1)-\pi(i) \geq l+2 f_{3}\left(3, k^{\prime}\right)$.

- There is a $\left(\mathcal{M}^{\prime \prime} \cup \Omega\right)$-path in $G-Z$ with two endpoints contained in different facial cycles of $C_{2 f_{3}\left(3, k^{\prime}\right)}\left[S_{\pi(i)-f_{3}\left(3, k^{\prime}\right)}^{\prime}\right]$. Moreover, two endpoints of this path is not contained in a single component of $\mathcal{M}^{\prime \prime}$.

Then 1 in Theorem E.1 holds.

Proof. By using the $\left(\mathcal{M}^{\prime \prime} \cup \Omega\right)$-path as in the third property, each $C_{2 f_{3}\left(3, k^{\prime}\right)}\left[S_{\pi(i)-f_{3}\left(3, k^{\prime}\right)}^{\prime}\right]$ contains one path $P_{1, \pi(i)-f_{3}\left(3, k^{\prime}\right)} \in \mathcal{M}^{\prime \prime}$ with endpoints $a, c$ and another path $P_{2, \pi(i)-f_{3}\left(3, k^{\prime}\right)}$ with endpoints $b, d$ such that $a, b, c, d \in \Omega$ appear in this order. Let us observe that any $\left(\mathcal{M}^{\prime \prime} \cup \Omega\right)$-path with two endpoints contained in different facial cycles of $C_{2 f_{3}\left(3, k^{\prime}\right)}\left[S_{\pi(j)-f_{3}\left(3, k^{\prime}\right)}^{\prime}\right]$ does not intersect any other $\left(\mathcal{M}^{\prime \prime} \cup \Omega\right)$-path with endpoints contained in different facial cycles of $C_{2 f_{3}\left(3, k^{\prime}\right)}\left[S_{\pi\left(j^{\prime}\right)-2 f_{3}\left(3, k^{\prime}\right)}^{\prime}\right]$ with $j \neq j^{\prime}$, otherwise there is a $\left(\mathcal{M}^{\prime \prime} \cup \Omega\right)$-path in $G-Z$ with distance $d_{\mathcal{M}^{\prime \prime} \cup \Omega}$ of two endpoints at least $f_{3}\left(3, k^{\prime}\right)$. Therefore, the paths $P_{2, \pi(i)-2 f_{3}\left(3, k^{\prime}\right)}$ does not intersect any other vertex in $P_{2, \pi\left(i^{\prime}\right)-2 f_{3}\left(3, k^{\prime}\right)}$ with $i \neq i^{\prime}$.

Thus we get a $(k, l)$-cross pattern or a $(k, l)$-twisted cross pattern in $G-Z$ by taking $P_{1, \pi(i)-f_{3}\left(3, k^{\prime}\right)}$ and $P_{2, \pi\left(i^{\prime}\right)-2 f_{3}\left(3, k^{\prime}\right)}$, together with components of $\mathcal{M}^{\prime \prime}$. 
Claim E.7 If there is an index $i$ such that the outer cycle of $C_{t}\left[S_{i}^{\prime}\right]$ is dividing in $G-Z$, and moreover the compass of $C_{t}\left[S_{i}^{\prime}\right]$ in $G-Z$ induces a 0-nearly flat decomposition, then 3 in Theorem E.1 holds.

Proof. If the assumption of Claim E.7 is satisfied, by the above remarks, we get a sublinkage $\hat{\mathcal{M}}^{\prime \prime}$ of $\mathcal{M}^{\prime \prime}$ such that $\hat{\mathcal{M}}^{\prime \prime}$ is either a $t$-handle or a $t$-crosscap. Moreover, except for one special facial cycle $F^{\prime}$, each facial cycle $F$ of $\hat{\mathcal{M}}^{\prime \prime}$ is dividing in $G-Z$, and in addition, the compass of $F$ in $G-Z$ induces a 0-nearly flat decomposition. It follows that 3 in Theorem E.1 holds with $Z$.

We pick up $C_{t}\left[S_{i}^{\prime}\right]$ for $\left.i=f_{3}\left(3, k^{\prime}\right), l+3 f_{3}\left(3, k^{\prime}\right), 2 l+5 f_{3}\left(3, k^{\prime}\right), \ldots, 2 k l+(2 \times 2 k+1) f_{3}\left(3, k^{\prime}\right)\right)$. If the outer cycle of $C_{t}\left[S_{i}^{\prime}\right]$ is not dividing in $G-Z$, then $C_{2 f_{3}\left(3, k^{\prime}\right)}\left[S_{i-f_{3}\left(3, k^{\prime}\right)}^{\prime}\right]$ contains a $\left(\mathcal{M}^{\prime \prime} \cup \Omega\right)$-path in $G-Z$ with two endpoints contained in different facial cycles of $C_{2 f_{3}\left(3, k^{\prime}\right)}\left[S_{i-f_{3}\left(3, k^{\prime}\right)}^{\prime}\right]$. Moreover, two endpoints of this path are not contained in a single component of $\mathcal{M}^{\prime \prime}$. Therefore, by our choice, either

- There are $k$ indices $\pi(1), \ldots, \pi(k)$ of $i$ such that $C_{t}\left[S_{\pi(i)}^{\prime}\right]$ is dividing in $G-Z$, or

- there are $k$ indices $\pi(1), \ldots, \pi(k)$ of $i$ such that $C_{2 f_{3}\left(3, k^{\prime}\right)}\left[S_{\pi(i)-f_{3}\left(3, k^{\prime}\right)}^{\prime}\right]$ contains a $\left(\mathcal{M}^{\prime \prime} \cup \Omega\right)$-path in $G-Z$ with two endpoints contained in different facial cycles of $C_{2 f_{3}\left(3, k^{\prime}\right)}\left[S_{\pi(i)-f_{3}\left(3, k^{\prime}\right)}^{\prime}\right]$. Moreover, two endpoints of this path is not contained in a single component of $\mathcal{M}^{\prime \prime}$.

For the second case, we are done by Claim E.6. Suppose the first case applies. By a result of Seymour [45], Thomassen [47], if Claim E.7 does not hold for any index, then it follows that the compass of the outer cycle of each $C_{t}\left[S_{\pi(i)}^{\prime}\right]$ in $G-Z$ contains two vertex-disjoint paths connecting the diagonally opposite corners of $C_{t}\left[S_{\pi(i)}^{\prime}\right]$ (that are vertices in $\Omega$ ). Thus we get 1 in Theorem E.1. This completes the proof of Theorem E.1.

\section{F Proof of the Structure Theorem}

In this section, we give the proof of the structure theorem, Theorem 2.1.

In the proof, we will apply the society theorem, Theorem A.6, and as a result, we have to analyze the case when we find a large leap pattern in a society. The leap pattern itself is slightly too general a structure to be useful. We begin this section with a short lemma for analyzing leap patterns. First, we state the following lemma which is a similar result to the lemma of Erdős and Szekeres.

Lemma F.1 Let $k \geq 1$ be a positive integer, let $(G, \Omega)$ be a society and let $\mathcal{M}$ be a pattern of order $k^{3}$. Then there exists a pattern $\mathcal{M}^{\prime}$ contained in $\mathcal{M}$ such that one of the following holds:
i. $\mathcal{M}^{\prime}$ is a k-crosscap;
ii. the elements of $\mathcal{M}^{\prime}$ are pairwise non-crossing and there exists two segments $S_{1}$ and $S_{2}$ of $\Omega$ such that every component of $\mathcal{M}^{\prime}$ has one endpoint in $S_{1}$ and one endpoint in $S_{2}$;
iii. $\mathcal{M}^{\prime}$ consists of $k$ independent $\Omega$-paths.

We now prove the following key lemma.

Lemma F.2 For all positive integers $k$ and $l$, there exist $k^{\prime}$ and $l^{\prime}$ such that the following holds. Let $(G, \Omega)$ be a society and $\mathcal{M}$ be a $\left(k^{\prime}, l^{\prime}\right)$ leap pattern in $(G, \Omega)$. Then there exists a pattern $\mathcal{M}^{\prime}$ contained in $\mathcal{M}$ such that:

$$
\text { 1. } \mathcal{M}^{\prime} \text { is a k-handle; }
$$




\section{2. $\mathcal{M}^{\prime}$ is a $k$-crosscap;}

3. $\mathcal{M}^{\prime}$ consists of the union of two disjoint patterns $\mathcal{P} \cup \mathcal{Q}$ such that $\mathcal{P}$ consists of $k$ independent $\Omega$-paths, and for every component of $P \in \mathcal{P}$, there exist $k$ distinct components of $\mathcal{Q}$ which cross $P$;

4. $\mathcal{M}^{\prime}$ is a $(k, l)$-cross pattern.

Proof. Let $\mathcal{M}=\mathcal{P} \cup \mathcal{Q}$ as in the definition of a leap pattern. We let $\bar{k}=\max \{k, l\}$, and we prove the theorem with $k^{\prime}=9 \bar{k}^{6}$ and $l^{\prime}=2 \bar{k}+1$. We apply Lemma F.1 to the pattern $\mathcal{Q}$. If $\mathcal{Q}$ contains a $\bar{k}$-crosscap, we satisfy outcome 2 . Also, note that for every component $Q \in \mathcal{Q}$, there exist $l^{\prime}$ distinct components of $\mathcal{P}$ which cross $Q$. Thus, if $\mathcal{Q}$ contains $\bar{k}$ independent $\Omega$-paths, then we satisfy outcome 3 .

We conclude that there exists a linkage $\mathcal{Q}^{\prime}$ of order $3 \bar{k}^{2}$ and segments $S_{1}$ and $S_{2}$ such that the components of $\mathcal{Q}^{\prime}$ are pairwise non-crossing and every component of $\mathcal{Q}^{\prime}$ has one endpoint in $S_{1}$ and the other endpoint in $S_{2}$. Label the components of $\mathcal{Q}^{\prime}$ as $Q_{1}, \ldots, Q_{3 \bar{k}^{2}}$, and let the endpoints of $Q_{i}$ be $s_{i}$ and $t_{i}$. We furthermore assume that $s_{i} \in S_{1}$ for all $1 \leq i \leq 3 \bar{k}^{2}$ and that $s_{1}, s_{2}, \ldots, s_{3 \bar{k}^{2}}$ occur on $S_{1}$ in that order. Note that, then $t_{3 \bar{k}^{2}}, t_{3 \bar{k}^{2}-1}, \ldots, t_{1}$ occur on $S_{2}$ in that order.

For all indices $i, \bar{k}+1 \leq i \leq 3 \bar{k}^{2}-\bar{k}$, consider the path $Q_{i}$. Let $X_{i}$ be the segment $s_{i+\bar{k}} \Omega t_{i+\bar{k}}$ containing $s_{i+\bar{k}+1}, \ldots, s_{3 \bar{k}^{2}}$. Similarly, define $Y_{i}$ to be the segment $s_{i-\bar{k}} \Omega t_{i-\bar{k}}$ which contains $s_{1}, \ldots, s_{i-\bar{k}+1}$. If there exist $\bar{k}$ components $P_{1}, \ldots, P_{\bar{k}}$ of $\mathcal{P}$ which all cross $Q_{i}$ and have one endpoint in $X_{i}$, then we see that $P_{1} \cup \cdots \cup P_{\bar{k}} \cup Q_{i} \cup Q_{i+1} \cup \cdots \cup Q_{i+\bar{k}-1}$ form a $\bar{k}$-handle in $(G, \Omega)$. Note, here we are using the property that the components of $\mathcal{P}$ are pairwise non-crossing. Alternatively, if there exist $\bar{k}$ components of $\mathcal{P}$ which cross $Q_{i}$ and have one endpoint $Y_{i}$, we similarly find a $\bar{k}$-handle in $(G, \Omega)$.

We conclude that for all $i, \bar{k}+1 \leq i \leq 3 \bar{k}^{2}-k$, there exists a component of $\mathcal{P}$, label it $P_{i}$, which crosses $Q_{i}$ and has both endpoints contained within $s_{i-\bar{k}} S_{1} s_{i+\bar{k}} \cup t_{i-\bar{k}} S_{2} t_{i+\bar{k}}$. The paths $\left\{P_{3 \bar{k} i-\bar{k}} \cup Q_{3 \bar{k} i-\bar{k}}: 1 \leq i \leq \bar{k}\right\}$ and $\bigcup_{i=1}^{\bar{k}-1} \bigcup_{j=1}^{\bar{k}} Q_{3 \bar{k} i+j}$ form a $(\bar{k}, \bar{k})$-cross pattern, proving the claim.

Before proceeding with the proof of the structure theorem, we give several additional lemmas. Let $(G, \Omega)$ be a society and $s \geq 1$ an integer. We say that $(G, \Omega)$ is $s$-nested if there exists a 1-nearly flat decomposition $H_{0}, H_{1}, \ldots, H_{n}$ of $(G, \Omega)$ such that all the small vortices are grounded, and for which there exists an $s$-nest $C_{1}, \ldots, C_{s}$ in $G$ such that $\Omega \subset V\left(C_{s}\right)$, and $H_{1}$ is contained in the disc $\Delta_{1}$ bounded by the cycle $C_{1}$.

We will also require the following three lemmas.

Lemma F.3 For any $t$, there is an integer $s_{1}$ such that if an $s_{1}$-nested society $(G, \Omega)$ contains either a $\left(4 t^{4}, 4 t^{4}\right)$-cross pattern $\mathcal{P}$ or a $\left(4 t^{4}, 4 t^{4}\right)$-twisted cross pattern $\mathcal{P}$, then $G$ has a $K_{t}-$ minor.

Proof. We first observe that both a $\left(4 t^{4}, 4 t^{4}\right)$-cross pattern $\mathcal{P}$ and a $\left(4 t^{4}, 4 t^{4}\right)$-twisted cross pattern $\mathcal{P}$ are crooked patterns of order at most $16 t^{12}$. Let $s_{1}=2 w\left(16 t^{12}\right)+16 t^{12}$, where $w($.$) is the function in Theorem$ C.1. We claim $s_{1}$ satisfies the theorem. It follows from Theorem C.2 (with $t$ replaced by $16 t^{12}$ ) that $(G, \Omega$ ) has a $16 t^{12}$-nest $C_{1}^{\prime}, \ldots, C_{16 t^{12}}^{\prime}$ and either a $\left(4 t^{4}, 4 t^{4}\right)$-cross pattern $\mathcal{P}^{\prime}$ or a $\left(4 t^{4}, 4 t^{4}\right)$-twisted cross pattern $\mathcal{P}^{\prime}$, which is equivalent to $\mathcal{P}$, such that $\mathcal{P}^{\prime}$ and $C_{1}^{\prime}, \ldots, C_{16 t^{12}}^{\prime}$ are orthogonal. It follows from Claims $33-35$ in [17] (and some similar configurations which we omit here because the proofs and constructions of them are essentially the same as Claims $33-35)$ that $G$ has a $K_{t}$-minor because $\mathcal{P}^{\prime}$ and $C_{1}^{\prime}, \ldots, C_{16 t^{12}}^{\prime}$ contain a " $16 t^{12}$-grid", and subpaths of $\mathcal{P}^{\prime}$ not in this grid form a matching after contracting edges incident vertices of degree two.

Lemma F.4 For any $t$, there are integers $k, l, s_{2}$ such that if an $s_{2}$-nested society $(G, \Omega)$ contains a pattern $\mathcal{P} \cup \mathcal{Q}$ such that $\mathcal{P}$ consists of $k$ independent $\Omega$-paths, and for every component of $P \in \mathcal{P}$, there exist $l$ distinct components of $\mathcal{Q}$ which cross $P$, then $G$ has a $K_{t}$-minor. 
Proof. We first observe that a pattern $\mathcal{P} \cup \mathcal{Q}$ is crooked and its order is $k+k l$. We take $N$ as in Lemma 32 in [17]. Moreover, we take $k, l$ such that $N \leq k l+k$, and moreover, $k, l$ satisfies $k, l$ in [17]. Let $s_{2}=w(k l+k)+N$, where $w($.$) is the function in Theorem C.1. We claim s_{2}$ satisfies the theorem. It follows from Theorem C.2 (with $t$ replaced by $N$ ) that $(G, \Omega)$ has an $N$-nest $C_{1}^{\prime}, \ldots, C_{N}^{\prime}$, and a pattern $\mathcal{P}^{\prime} \cup \mathcal{Q}^{\prime}$, which is equivalent to $\mathcal{P} \cup \mathcal{Q}$, such that $\mathcal{P}^{\prime} \cup \mathcal{Q}^{\prime}$ and $C_{1}^{\prime}, \ldots, C_{N}^{\prime}$ are orthogonal. It follows from Lemma 32 in [17] that $G$ has a $K_{t}$-minor because $\mathcal{P}^{\prime} \cup \mathcal{Q}^{\prime}$ and $C_{1}^{\prime}, \ldots, C_{N}^{\prime}$ contain an " $N$-grid", and, again, the subpaths of $\mathcal{P}^{\prime} \cup \mathcal{Q}^{\prime}$ not in this grid form a matching after contracting edges incident vertices of degree two.

Lemma F.5 For any $t$, there are integers $k, l, s_{3}$ such that the following holds. If an $s_{3}$-nested society $(G, \Omega)$ contains a segment $S$ of $\Omega$ such that if we let $\Omega^{\prime}$ be the cyclic order of $\Omega \backslash S$ induced by $\Omega$, then there exists a pattern $\mathcal{P} \cup \mathcal{Q}$ in $\left(G, \Omega^{\prime}\right)$ such that $\mathcal{P}$ consists of $k$ independent $\Omega$-paths $P_{1}, \ldots, P_{k}$ and for every component of $P \in \mathcal{P}$, there exist $l$ distinct components of $\mathcal{Q}$ which cross $P$. Then $G$ has a $K_{t}$-minor.

Proof. We take $k, l, N$ as in the proof of Lemma F.4, and $N^{\prime}=N+1, s_{3}=w(k+k l)+N^{\prime}$. We claim $s_{3}$ satisfies the theorem. By possibly sacrificing the cycle $C_{1}$, we find a new pattern $\mathcal{P}^{\prime} \cup \mathcal{Q}^{\prime}$ in $(G, \Omega)$, such that $\mathcal{P}^{\prime}$ consists of $k$ independent $\Omega$-paths, and for every component of $P \in \mathcal{P}^{\prime}$, there exist $l$ distinct components of $\mathcal{Q}^{\prime}$ which cross $P$. Therefore, we can reduce to Lemma F.4 with $s_{2}=s_{3}$.

Finally, we are ready to finish our proof of Theorem 2.1. We proceed step by step to emphasize how an algorithm can be extracted from the proof.

Assume that we are given a graph $G$, and $R=K_{t}$. Assume that a tangle $\mathcal{T}$ of order $\Theta$ is given. We will specify $\Theta$ and $\alpha=\alpha(t)$ later.

Step 1. Finding a large wall $W$, controlled by the tangle $\mathcal{T}$. We first apply Theorem A.2 to obtain a wall $W$ of size $q(t, r)$, controlled by this tangle $\mathcal{T}$ (where $q(t, r)$ comes from Theorem A.7, and $r$ will be determined later). Note that we can find such a wall $W$ in $O(n)$ time as in Theorem A.2.

We assume $\Theta \geq f_{2}(q(t, r))$, where $f_{2}(),. q(.,$.$) come from Theorems A.2 and A.7, respectively. We also$ assume $r>3 \alpha$.

This wall $W$ is one of the keys. Specifically, for each separation $(A, B)$ of order at most $3 \alpha$, we are interested in the "big" side $B$. This wall $W$ gives a certificate which side is "big", because $B$ always contains all but at most $3 \alpha$ nails of $W$. In other words, $A$ contains at most $3 \alpha$ nails of $W$. Therefore the wall $W$ always indicates which side is "big" for each separation of order $3 \alpha$.

Step 2. Applying the Weak Structure Theorem to the wall $W$. We now apply Theorem A.7 with this wall $W$. Thus either $G$ has a $K_{t}$-minor, controlled by the tangle $\mathcal{T}$ (as remarked just after Theorem A.7), or else there is a vertex set $A$ of order $t^{2}$, such that $G-A$ can be decomposed into subgraphs $H$ and $G^{\prime}$ with $G-A=G^{\prime} \cup H$. Moreover, the graph $H$ has a flat $r$-wall decomposition $H_{0}, H_{1}, \ldots, H_{n}$, a subwall $W^{\prime}$ of size $r$ of $W$, and a boundary cycle $C$ satisfying:

i) $\partial_{G-A}\left(V\left(G^{\prime}\right)\right) \subseteq V(C) \cap V\left(H_{0}\right)$, and

ii) every vertex of degree 3 in $W^{\prime}$ is contained in $V\left(H_{0}\right)$.

Thus for each $i \geq 1, H_{i} \cup A$ is a small side in the separation $\left(H_{i} \cup A, G-\left(H_{i}-H_{0}\right)\right)$, with respect to the tangle $\mathcal{T}$. The value $r$ will be determined later. This structure can be obtained in $O(n)$ time, see [20]. 
Step 3. Growing a surface. In this step, we give the setting for our induction. We first let $k, l$ be as in Lemmas F.4 and F.5. We also take $s_{1} \leq s_{2} \leq s_{3}$ as in Lemmas F.3, F.4 and F.5. We introduce two parameters $g_{1}(i), g_{2}(i)$ as follows.

1. $g_{1}(i)$ is an increasing function such that $g_{1}(0)=t^{2}$, and $g_{1}(i)=g_{1}(i-1)+a(k, l)+g_{2}(1)$, where $a(k, l)$ comes from Theorem E.1 and $g_{2}(i)$ is as below;

2. $g_{2}(i)$ is a decreasing function with $i \leq t^{2}$, such that $g_{2}\left(t^{2}\right)=f^{\prime}(t, \Sigma)$, where $f^{\prime}(t, \Sigma)$ comes from Theorem A.8. Moreover, $g_{2}(i)=2 w\left(f\left(2 g_{2}(i+1)+a(k, l), k\right)\right)+2\left(2 g_{2}(i+1)+a(k, l)+s_{3}\right)$, where $w(),. f(.,$.$) come from Theorem C.2 and Theorem E.1, respectively.$

We also set $r \geq 2 g_{2}(0) \geq 2^{t^{2}} f^{\prime}(t, \Sigma)$. Intuitively, $g_{1}(i)$ is a bound for the number of apex vertices while $g_{2}(i)$ is a bound for the representativity, for the step $i$.

Let $H_{i}, F_{i}, Z_{i}, G_{i}$ and $\Sigma_{i}$ be the following.

i. $G$ has a vertex set $Z_{i}$ with $\left|Z_{i}\right| \leq g_{1}(i)$.

ii. $G-Z_{i}=H_{i} \cup G_{i}$ and $H_{i} \cap G_{i}=F_{i}$.

iii. $H_{i}$ has a 0 -nearly flat embedding in $\Sigma_{i}$ of euler genus at least $i$, i.e, $H_{i}$ consists of edge-disjoint subgraphs $H_{i, 0}, \ldots, H_{i, m}$ such that

1. $\bigcup_{j=0}^{m} H_{i, j}=H_{i}$,

2. for all $j_{1}, j_{2} \geq 1, j_{1} \neq j_{2}, V\left(H_{i, j_{1}}\right) \cap V\left(H_{i, j_{2}}\right) \subseteq V\left(H_{i, 0}\right)$,

3. for all $j>0,\left|V\left(H_{i, 0}\right) \cap V\left(H_{i, j}\right)\right| \leq 3$, and

4. there exist pairwise disjoint open discs $\Delta_{i, 1}, \ldots, \Delta_{i, m}$ and an embedding $\sigma: H_{i, 0} \hookrightarrow \Sigma_{i}-\bigcup_{j=1}^{m} \Delta_{i, j}$ such that the only vertices contained in the boundary of $\Delta_{i, j}$ are exactly the vertices of $H_{i, j} \cap H_{i, 0}$.

$i v$. For each $j \geq 1, H_{i, j} \cup Z_{i}$ is a small side in the separation $\left(H_{i, j} \cup Z_{i}, G-\left(H_{i, j}-H_{i, 0}\right)\right)$ with respect to the tangle $\mathcal{T}$.

$v$. If $i>0$, then the embedding of $\bar{H}_{i, 0}$ in $\Sigma_{i}$ has representativity at least $g_{2}(i)$. If $i=0$, then $\bar{H}_{0,0}$ contains a wall of size $g_{2}(0)$. Moreover, $F_{i}$ bounds a disc in the embedding of $\bar{H}_{i, 0}$ in $\Sigma_{i}$.

$v i$. Let $\Omega_{i}$ be $F_{i}$ with the natural cyclic ordering of $F_{i}$. Then $\left(G_{i}, \Omega_{i}\right)$ is a $g_{2}(i) / 2$-nested society.

We first show that such a choice $i$ satisfying $i$. $-v i$. is possible. More precisely;

Claim If $i=0$, then there are desired $H_{0}, F_{0}, Z_{0}, G_{0}$ and $\Sigma_{0}$ satisfying $i .-v i$. .

Subproof. By Step 2, we have that the vertex set $A$ which will correspond to $Z_{0}$. We assume the notations given in Step 2. Observe that the notations in Step 2 and iii. actually match.

We pick a $g_{2}(0) / 2$-nest $C_{1}, \ldots, C_{g_{2}(0) / 2}$ in $H$, where $C=C_{1}$, so that $H_{0}=H-\Delta_{g_{2}(0) / 2}$ still has a wall of size $g_{2}(0)$, where $\Delta_{j}$ is the disc bounded by $C_{j}$ such that $\Delta_{g_{2}(0) / 2} \supseteq \ldots, \Delta_{1}$. Such a choice is clearly possible because $H$ contains a flat $r$-wall with $r \geq 2 g_{2}(0)$. Let $F_{0}=V\left(\bar{C}_{g_{2}(0) / 2}\right)$ and $G_{0}=G^{\prime} \cup \Delta_{g_{2}(0) / 2} \cup F_{0}$. Let $\Omega_{0}$ be $F_{0}$ with the natural cyclic ordering of $F_{0}$. Then $\left(G_{0}, \Omega_{0}\right)$ is a $g_{2}(0) / 2$-nested society. Clearly for each $j \geq 1, H_{0, j} \cup Z_{0}$ is a small side in the separation $\left(H_{0, j} \cup Z_{0}, G-\left(H_{0, j}-H_{0,0}\right)\right)$ with respect to the tangle $\mathcal{T}$, because $H_{0,0}$ contains many more than $3 \alpha$ nails of $W$. Thus $H_{0}, F_{0}, Z_{0}, G_{0}$ and $\Sigma_{0}$ satisfy $i-v i$. This proves the claim.

Let us observe that the structure in Step 3 with $i=0$ can be easily found in $O(n)$ time once the structure as in the weak structure theorem A.7 is given (which is done in Step 2).

Note that $H_{i}, F_{i}, Z_{i}$ and $\Sigma_{i}$ are the structures that appear in Theorem 2.1. What remains is to analyze the society $\left(G_{i}, \Omega_{i}\right)$. Thus, the remainder of the proof is to analyze the structure of the society.

We pick an index $i$ and $H_{i}, F_{i}, Z_{i}, G_{i}$ and $\Sigma_{i}$ satisfying $i-v i$ with the value $i$ to be maximal. 
Step 4. Applying the society theorem to the society $\left(G_{i}, \Omega_{i}\right)$. From Step 4 to Step 6, we shall work on the society $\left(G_{i}, \Omega_{i}\right)$. Let $k_{1}=2 g_{2}(i+1)+a(k, l)$. We apply Theorem A.6 to the $g_{2}(i) / 2$-nested society $\left(G_{i}, \Omega_{i}\right)$ with $\left.\hat{k}=k^{\prime}\left(k_{1}, k\right)\right), \hat{l}=l^{\prime}(l)\left(k^{\prime}(\right.$.$) and l^{\prime}($.$) come from Lemma F.2, and f(.,$.$) comes from Theorem$ E.1). If the outcome is 2 , then we get the desired structure as in Theorem 2.1, with $\alpha(t)=\alpha(\hat{k}, \hat{l})$, where $\alpha(\hat{k}, \hat{l})$ is as in Theorem A.6.

Suppose the outcome is 1 . If we get a $\left(k^{\prime}, l^{\prime}\right)$-cross pattern, then by Lemma F.3, $G_{i}$ has a $K_{t}$-minor (which is actually controlled by the tangle $\mathcal{T}$, as easily seen). If we get a $k^{\prime}\left(f\left(k_{1}, k\right)\right)$-crosscap, we go to Step 6 . If we get a $\left(k^{\prime}\left(f\left(k_{1}, k\right)\right), l^{\prime}(l)\right)$-leap pattern, then we go to Step 5.

Let us observe that the structure in Step 4 can be found by the constructive proof of Theorem A.6, which can be converted in an $O\left(n^{2}\right)$ time algorithm.

Step 5. Analyzing a $\left(k^{\prime}\left(f\left(k_{1}, k\right)\right), l^{\prime}(l)\right)$-leap pattern in the society $\left(G_{i}, \Omega_{i}\right)$. At the moment, there is a $\left(k^{\prime}\left(f\left(k_{1}, k\right)\right), l^{\prime}(l)\right)$-leap pattern in the $g_{2}(i) / 2$-nested society $\left(G_{i}, \Omega_{i}\right)$. We apply Lemma F.2 to this $\left(k^{\prime}\left(f\left(k_{1}, k\right)\right), l^{\prime}(l)\right)$-leap pattern in the $g_{2}(i) / 2$-nested society $\left(G_{i}, \Omega_{i}\right)$. If we get either a $f\left(k_{1}, k\right)$-crosscap or a $f\left(k_{1}, k\right)$-handle, then we go to Step 6 .

Suppose the third outcome of Lemma F.2 happens. Thus the $g_{2}(i) / 2$-nested society $\left(G_{i}, \Omega_{i}\right)$ contains a pattern $\mathcal{P} \cup \mathcal{Q}$ such that $\mathcal{P}$ consists of $k$ independent $\Omega$-paths, and for every component of $P \in \mathcal{P}$, there exist $l \geq k$ distinct components of $\mathcal{Q}$ which cross $P$. Then by Lemma F.4, $G_{i}$ has a $K_{t}$-minor (which can be shown to be controlled by the tangle $\mathcal{T}$ ).

Finally, if we get a $(k, l)$-cross pattern in the $g_{2}(i) / 2$-nested society $\left(G_{i}, \Omega_{i}\right)$, then by Lemma F.3, $G_{i}$ has a $K_{t}$-minor (which is actually controlled by the tangle $\mathcal{T}$, as easily seen).

Let us observe that the structure in Step 5 can be found by the constructive proof of Lemma F.2, which can be converted in an $O(n)$ time algorithm. Moreover, $K_{t}$-minors in Lemmas F.3 and F.4 can be constructed in $O(n)$ time by following the proofs.

Step 6. Finding a new society in the society $\left(G_{i} \Omega_{i}\right)$. At the moment, the $g_{2}(i) / 2$-nested society $\left(G_{i}, \Omega_{i}\right)$ contains either a $f\left(k_{1}, k\right)$-crosscap $\mathcal{P}$ or a $f\left(k_{1}, k\right)$-handle $\mathcal{P}$. By Theorem C.2, $\left(G_{i}, \Omega_{i}\right)$ has either a $f\left(k_{1}, k\right)$-crosscap $\mathcal{P}^{\prime}$ or a $f\left(k_{1}, k\right)$-handle $\mathcal{P}^{\prime}$, which is equivalent to $\mathcal{P}$, and a $\left(k_{1}+s_{3}\right)$-nest $C_{1}, \ldots, C_{k_{1}+s_{3}}$, such that $\mathcal{P}^{\prime}$ and $C_{1}, \ldots, C_{k_{1}+s_{3}}$ are orthogonal. Note that $s_{3}$ comes from Lemma F.3.

Let $G_{i}^{\prime}=\Delta_{s_{3}} \cup \bar{C}_{s_{3}}$, and let $\Omega_{i}^{\prime}$ be $V\left(\bar{C}_{s_{3}}\right)$ with the natural cyclic order of the cycle $\bar{C}_{s_{3}}$. So $G_{i}^{\prime}$ contains the graph contained in the disc $\Delta_{s_{3}}$. Thus $\left(G_{i}^{\prime}, \Omega_{i}^{\prime}\right)$ is the $s_{3}$-nested society. Let $\mathcal{P}^{\prime \prime}$ be the pattern of $\mathcal{P}^{\prime}$ induced by $\left(G_{i}^{\prime}, \Omega_{i}^{\prime}\right)$. Thus $\mathcal{P}^{\prime \prime}$ is either a $f\left(k_{1}, k\right)$-crosscap pattern or a $f\left(k_{1}, k\right)$-handle pattern.

Let the annulus bounded by $C_{k_{1}+s_{3}}$ and $C_{s_{3}}$ be $Q_{i}$. This $Q_{i}$ will be used to maintain the representativity of $H_{i+1,0}$ when we construct $H_{i+1}, F_{i+1}, Z_{i+1}, G_{i+1}$ and $\Sigma_{i+1}$. Note that the annulus $Q_{i}$ has $f\left(k_{1}, k\right)$ disjoint paths obtained by the pattern $\mathcal{P}^{\prime}$ in $Q_{i}$, such that they are orthogonal to the $k_{1}$-nest $C_{s_{3}}, \ldots, C_{k_{1}+s_{3}}$ in $Q_{i}$.

We apply Theorem E.1 to the $s_{3}$-nested society $\left(G_{i}^{\prime}, \Omega_{i}^{\prime}\right)$ with either a $f\left(k_{1}, k\right)$-crosscap pattern $\mathcal{P}^{\prime \prime}$ or a $f\left(k_{1}, k\right)$-handle pattern $\mathcal{P}^{\prime \prime}$. If we get a $(k, l)$-cross pattern or a $(k, l)$-twisted cross pattern in the $s_{3}$-nested society $\left(G_{i}^{\prime}, \Omega_{i}^{\prime}\right.$ ), then by Lemma F.3, $G_{i}^{\prime}$ has a $K_{t}$-minor (which is controlled by the tangle $\mathcal{T}$, as can be easily seen). Thus the first outcome of Theorem E.1 cannot happen.

Suppose the second outcome of Theorem E.1 happens. Thus there exists a segment $S$ of $\Omega_{i}^{\prime}$ such that if we let $\Omega^{\prime \prime}$ be the cyclic order of $\Omega_{i}^{\prime} \backslash S$ induced by $\Omega_{i}^{\prime}$, then there exists a pattern $\mathcal{P} \cup \mathcal{Q}$ in $\left(G_{i}^{\prime}, \Omega^{\prime \prime}\right)$ such that $\mathcal{P}$ consists of $k$ independent $\Omega_{i}^{\prime}$-paths $P_{1}, \ldots, P_{k}$ and for every component of $P \in \mathcal{P}$, there exist $l$ distinct components of $\mathcal{Q}$ which cross $P$. Then by Lemma F.5, $G_{i}^{\prime}$ has a $K_{t}$-minor (which again must be controlled by the tangle $\mathcal{T}$ ).

Finally, suppose the third outcome happens. Thus, after deleting at most $a(k, l)$ vertices (call the set 
$Z^{\prime}$ ), and adding $Z^{\prime}$ to $Z_{i}$ (and let $Z_{i+1}$ be the resulting vertex set), there is a pattern $\hat{\mathcal{P}}$ in the society $\left(G_{i}^{\prime}-Z_{i+1}, \Omega_{i}^{\prime}-Z_{i+1}\right)$, which is obtained from $\mathcal{P}^{\prime}$ induced by $G_{i}^{\prime}-Z_{i+1}$, such that $\hat{\mathcal{P}}$ is either a $\left(k_{1}-a(k, l)\right)$ crosscap or a $\left(k_{1}-a(k, l)\right)$-handle. Moreover, if we embed this pattern $\hat{\mathcal{P}}$ into the disc bounded by $\hat{C}_{s_{3}}$, then there is one "special" face $F$ in the embedding in such a way that $G_{i}^{\prime}-Z_{i+1}-\Delta F$ has a 0 -nearly flat decomposition. Note that the annulus $Q_{i}$ has $2 g_{2}(i+1)$-paths, which can connect the pattern $\hat{\mathcal{P}}$ to obtain the pattern $\hat{\mathcal{P}}^{\prime}$ for the society $\left(G_{i}-Z_{i+1}, \Omega_{i}-Z_{i+1}\right)$. Note that the pattern $\hat{\mathcal{P}}^{\prime}$ is either a $2 g_{2}(i+1)$-crosscap or a $2 g_{2}(i+1)$-handle. Moreover, they are orthogonal to the $2 g_{2}(i+1)$-nest in $Q_{i}$, too.

Let $H_{i}^{\prime}=H_{i} \cup\left(G_{i}-\Delta F-Z_{i+1}\right)$. We claim that $H_{i}^{\prime}$ has a 0 -nearly flat embedding in $\Sigma_{i+1}$ of euler genus at least $i+1$ such that representativity of $\hat{H}_{i, 0}^{\prime}$ is at least $2 g_{2}(i+1)$. It is clear that $H_{i}^{\prime}$ has a 0-nearly flat embedding in $\Sigma_{i+1}$ of euler genus at least $i+1$. It remains to show that representativity of $\hat{H}_{i, 0}^{\prime}$ is at least $2 g_{2}(i+1)$. It is easy to see that the pattern $\hat{\mathcal{P}}^{\prime}$, together with the $2 g_{2}(i+1)$-nest in $Q_{i}$, can be embedded on a torus or a projective plane with representativity at least $2 g_{2}(i+1)$. We claim that there is no contractible curve $C^{\prime}$ of order at most $2 g_{2}(i+1)$ in $\hat{H}_{i, 0}^{\prime}$, such that the disc bounded by the curve $C^{\prime}$, together with $V\left(C^{\prime}\right)$, contains the face $F$. Otherwise, we delete the vertices (call $T$ ) that hit the curve $C^{\prime}$, and add $T$ to $Z_{i+1}$. Then we have a resulting graph $H_{i+1}$ of $H_{i}^{\prime}$ that has a 0-nearly flat embedding in $\Sigma_{i}$ of euler genus at least $i$, such that $H_{i+1}, Z_{i+1}$ and $\Sigma_{i}$ satisfy the structure in Theorem 2.1. Note that in this case, there are no $F_{i+1}$ nor $G_{i+1}$. Clearly for each $j \geq 1, H_{i+1, j} \cup Z_{i+1}$ is a small side in the separation $\left(H_{i+1, j} \cup Z_{i+1}, G-\left(H_{i+1, j}-H_{i+1,0}\right)\right)$ with respect to the tangle $\mathcal{T}$, because $H_{i+1,0}$ contains much more than $3 \alpha$ nails of $W$. Hence we are done. Thus we do not have such a contractible curve, which means that we can maintain representativity of $H_{i, 0}^{\prime}$ that is at least $2 g_{2}(i+1)$, as claimed.

Since $H_{i}^{\prime}$ has a 0 -nearly flat embedding in $\Sigma_{i+1}$ of euler genus at least $i+1$ and representativity of $\bar{H}_{i, 0}^{\prime}$ is at least $2 g_{2}(i+1)$, we can take $g_{2}(i+1) / 2$ nested cycles $C_{1}^{\prime}, \ldots, C_{g_{2}(i+1) / 2}^{\prime}$ in $H_{i}^{\prime}$ so that $C_{1}^{\prime}=F$ and the embedding of $\bar{H}_{i, 0}^{\prime}-\Delta_{g_{2}(i+1) / 2}$ has representativity at least $g_{2}(i+1)$ in $\Sigma_{i+1}$ (see [26]). Let $G_{i+1}$ be the graph contained in $\Delta_{g_{2}(i+1) / 2} \cup C_{g_{2}(i+1) / 2}^{\prime}$. Then $\left(G_{i+1}, \Omega_{i+1}\right)$ is a $g_{2}(i+1) / 2$-nested society such that $\Omega_{i+1}=V\left(\bar{C}_{g_{2}(i+1) / 2}^{\prime}\right)$ and the cyclic order of $\Omega_{i+1}$ is the natural way of $\bar{C}_{g_{2}(i+1) / 2}^{\prime}$. Let $F_{i+1}=C_{g_{2}(i+1) / 2}^{\prime}$.

Thus we get $H_{i+1}, F_{i+1}, Z_{i+1}, G_{i+1}$ and $\Sigma_{i+1}$ with $H_{i+1}=H_{i}^{\prime}-\Delta_{g_{2}(i+1) / 2}$ and $F_{i+1}=V\left(\bar{C}_{1 / 2 g_{2}(i+1)}^{\prime}\right)$. Clearly for each $j \geq 1, H_{i+1, j} \cup Z_{i+1}$ is a small side in the separation $\left(H_{i+1, j} \cup Z_{i+1}, G-\left(H_{i+1, j}-H_{i+1,0}\right)\right)$ with respect to the tangle $\mathcal{T}$, because $H_{i+1,0}$ contains much more than $3 \alpha$ nails of $W$. This contradicts the maximality of $i$. Note that if $i \geq t^{2}$, then we can find a $K_{t}$-minor by Theorem A.8. This completes the proof of Theorem 2.1.

Let us observe that the structure in Step 6 can be found by the constructive proof of Theorem E.1, which can be found in time $O\left(n^{2}\right)$. Moreover, the $K_{t}$-minor in Lemma F.5 can be constructed in $O(n)$ time by following the proof. Since we know the bound $i \leq t^{2}$, thus we have at most $t^{2}$ iterations to perform Steps 4-6. We conclude that we construct the structure desired in Theorem 2.1 in $O\left(n^{2}\right)$ time,

Finally, it follows from our proof that we can get slightly stronger conclusions than Theorem 2.1. Namely, either we can find a $K_{t}$-minor, controlled by the tangle $\mathcal{T}$, or we can get the structure as in Theorem 2.1. Moreover, given the inputs as in Theorem 2.1 with $R=K_{t}$ for fixed $t$, we can reach one of these two conclusions in $O\left(n^{2}\right)$ time for fixed $t$. 